\title{
Supplementary Data for: Electrochemical oxygen reduction at soft interfaces catalyzed by the transfer of hydrated lithium cations
}

Haiqiang Deng, ${ }^{\mathrm{a}}$ T. Jane Stockmann, ${ }^{\mathrm{a}}$ Pekka Peljo, ${ }^{\mathrm{a}}$ Marcin Opallo, ${ }^{\mathrm{b}}$ and Hubert H. Girault ${ }^{\mathrm{a}, \dagger}$

aLaboratoire d'Electrochimie Physique et Analytique, Ecole Polytechnique Fédérale de Lausanne (EPFL), Station 6, CH-1015 Lausanne, Switzerland

bInstitute of Physical Chemistry, Polish Academy of Sciences, ul. Kasprzaka 44/52, 01-224

Warszawa, Poland

${ }^{\dagger}$ To whom correspondence should be addressed. Tel: +41-21-693 3145; Fax: +41-21-693 3667;

Email: hubert.girault@epfl.ch

URL: http://lepa.epfl.ch/

Table of Contents:

1. Table S1: COMSOL simulation parameters

2. Figure S1: Modifying the ratio of $k_{c} f k_{c b}$

3. COMSOL Model Report 
Table S1: COMSOL simulation parameters

\begin{tabular}{|c|c|c|}
\hline Parameter & Value & Description \\
\hline$v$ & $0.050 \mathrm{~V} \cdot \mathrm{s}^{-1}$ & Scan rate \\
\hline$\Delta_{o}^{w} \phi_{i}$ & $0.000 \mathrm{~V}$ & Initial potential \\
\hline$\Delta_{o}^{w} \phi_{f}$ & $0.550 \mathrm{~V}$ & Upper potential \\
\hline$\Delta_{o}^{w} \phi_{\mathrm{Li}^{+}}^{o^{\prime}}$ & $0.696 \mathrm{~V}$ & Formal Li ${ }^{+}$transfer potential (ref. [1]) \\
\hline$\Delta_{o}^{w} \phi_{\mathrm{OH}^{-}}^{o^{\prime}}$ & $-0.696 \mathrm{~V}$ & Formal $\mathrm{OH}^{-}$transfer potential * \\
\hline$\Delta_{o}^{w} \phi_{\mathrm{DMFc}^{+}}^{o^{\prime}}$ & $-0.250 \mathrm{~V}$ & Formal DMFc ${ }^{+}$transfer potential \\
\hline$k^{0}$ & $0.01 \mathrm{~m} \cdot \mathrm{s}^{-1}$ & Standard rate constant \\
\hline$\alpha$ & 0.5 & Transfer coefficient \\
\hline$c_{\mathrm{M}^{+}, w} / c_{\mathrm{M}^{+}, 0}$ & $10 \mathrm{mmol} \cdot \mathrm{L}^{-1} / 0 \mathrm{mmol} \cdot \mathrm{L}^{-1}$ & Initial metal ion concentration \\
\hline$c_{\mathrm{DMFc}, w} / C_{\mathrm{DMF}, \mathrm{o}}$ & $0 \mathrm{mmol} \cdot \mathrm{L}^{-1} / 5 \mathrm{mmol} \cdot \mathrm{L}^{-1}$ & Initial concentration of DMFc \\
\hline$c_{\mathrm{OH}^{-}, w} / c_{\mathrm{OH}^{-}, 0}$ & $10 \mathrm{mmol} \cdot \mathrm{L}^{-1} / 0 \mathrm{mmol} \cdot \mathrm{L}^{-1}$ & Initial hydroxide concentration \\
\hline$c_{\mathrm{O}_{2}, \mathrm{o}}$ & $1.6 \mathrm{mmol} \cdot \mathrm{L}^{-1}$ & Initial oxygen concentration (ref. [2-4]) \\
\hline$D_{\mathrm{DMFc}}$ & $7.26 \times 10^{-6} \mathrm{~cm} \cdot \mathrm{s}^{-1}$ & $\begin{array}{l}\text { Diffusion coefficient of DMFc and DMFc } \\
\text { (ref. [5]) }\end{array}$ \\
\hline$D_{\mathrm{O}_{2}}$ & $2.76 \times 10^{-5} \mathrm{~cm} \cdot \mathrm{s}^{-1}$ & Diffusion coefficient of $\mathrm{O}_{2}$ (ref. [2]) \\
\hline$D_{\mathrm{M}^{+}}$ & $1.0 \times 10^{-5} \mathrm{~cm} \cdot \mathrm{s}^{-1}$ & Diffusion coefficient of $\mathrm{M}^{+}$ \\
\hline$D_{\mathrm{OH}^{-}}$ & $1.0 \times 10^{-5} \mathrm{~cm} \cdot \mathrm{s}^{-1}$ & Diffusion coefficient of $\mathrm{OH}^{-}$ \\
\hline$k_{c f 2} / k_{c b 2}(\mathrm{org})$ & $5000 \mathrm{~L} \cdot \mathrm{mol}^{-1} \cdot \mathrm{s}^{-1} / 1 \mathrm{~s}^{-1}$ & Rate of ion-pair formation in the organic phase \\
\hline$k_{c f 2} / k_{c b 2}(a q)$ & $1 \mathrm{~L} \cdot \mathrm{mol}^{-1} \cdot \mathrm{s}^{-1} / 1 \times 10^{5} \mathrm{~s}^{-1}$ & $\begin{array}{l}\text { Rate of ion-pair formation in the aqueous } \\
\text { phase }\end{array}$ \\
\hline$r_{d}$ & $0.7 \mathrm{~cm}$ & Radius of the ITIES \\
\hline
\end{tabular}

*A value of $-0.656 \mathrm{~V}$ is described in the literature (ref. [6]) for $\Delta_{o}^{w} \phi_{\mathrm{OH}^{-}}^{o^{\prime}}$; however, to make the simulated CVs symmetric, and for the sake of convenience, $-0.696 \mathrm{~V}$ was employed. 


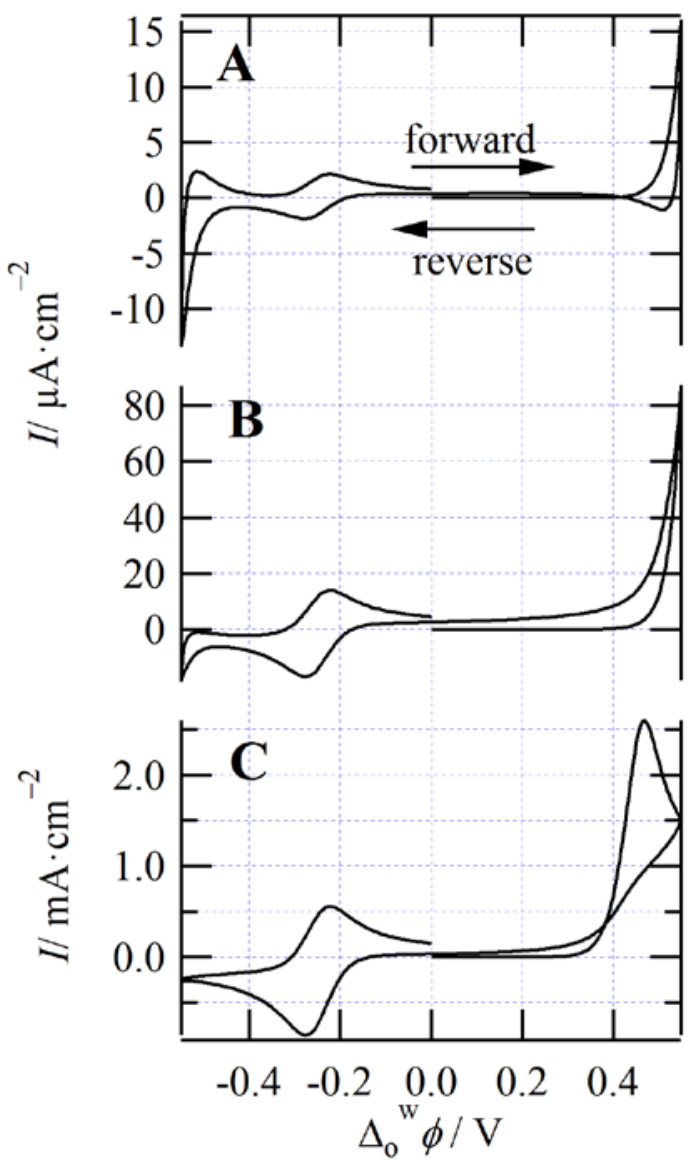

Figure S1: Simulated cyclic voltammograms compiled using the simulation detailed in section 2.0 of the main text with similar parameters as provided for Figure 4 and Table S1; however, with $k_{\text {chem-1 }}, k_{\text {chem-2 }}, k_{\text {chem-3 }}$, and $k_{\text {chem-4 }}$ equal to $1 \times 10^{4} \mathrm{~L} \cdot \mathrm{mol}^{-1} \cdot \mathrm{s}^{-1}$, while $k_{c f} / k_{c b}$ were changed to

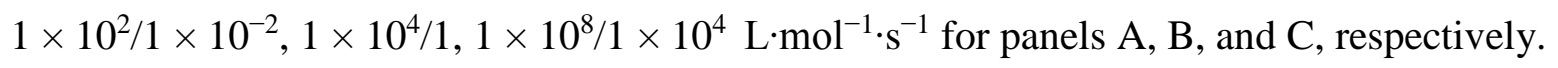

\section{References}

[1] T.J. Stockmann, A.-M. Montgomery, Z. Ding, J. Electroanal. Chem. 684 (2012) 6-12.

[2] A. Schumpe, P. Luehring, J. Chem. Eng. Data 35 (1990) 24-5.

[3] A. Trojánek, J. Langmaier, Z. Samec, Electrochem. Commun. 8 (2006) 475-81.

[4] P. Luehring, A. Schumpe, J. Chem. Eng. Data 34 (1989) 250-2.

[5] T.J. Stockmann, H. Deng, P. Peljo, K. Kontturi, M. Opallo, H. Girault, J. Electroanal. Chem. (2014) Submitted.

[6] M. Zhou, S. Gan, L. Zhong, X. Dong, J. Ulstrup, D. Han, L. Niu, Phys. Chem. Chem. Phys. 14 (2012) 3659-68. 


\section{MULTIPHツSICS 3 Contents}

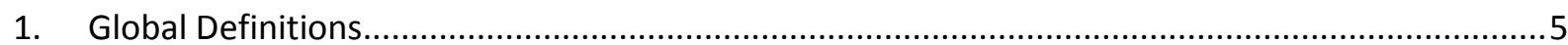

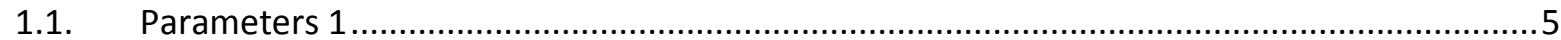

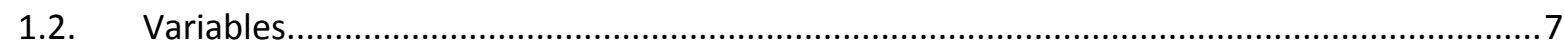

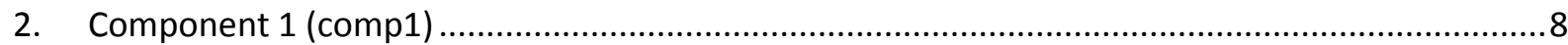

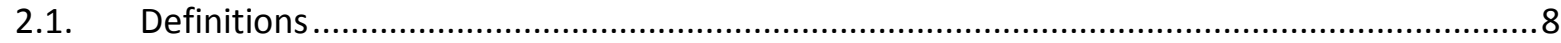

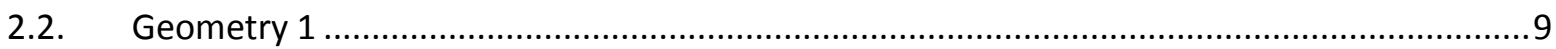

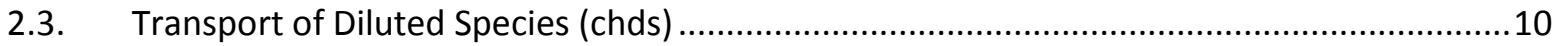

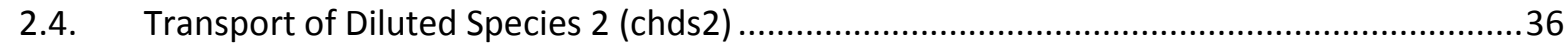

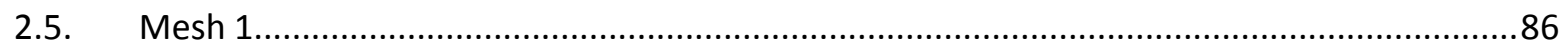




\section{Global Definitions}

\subsection{Parameters 1}

Parameters

\begin{tabular}{|l|l|l|}
\hline Name & Expression & Description \\
\hline F & $96485[\mathrm{C} / \mathrm{mol}]$ & \\
\hline T & $298.15[\mathrm{~K}]$ & \\
\hline R & $8.314[\mathrm{~J} / \mathrm{mol} / \mathrm{K}]$ & \\
\hline fara & F/(R*T) & \\
\hline Ei & $0[\mathrm{~V}]$ & \\
\hline Ef & $0.55[\mathrm{~V}]$ & \\
\hline E0 & $0.696[\mathrm{~V}]$ & formal IT of $\mathrm{M}+$ \\
\hline E02 & $-0.25[\mathrm{~V}]$ & formal IT of DMFc+ \\
\hline E03 & $-0.696[\mathrm{~V}]$ & formal IT potential for OH- \\
\hline nu & $0.050[\mathrm{~V} / \mathrm{s}]$ & \\
\hline k0 & $1[\mathrm{~cm} / \mathrm{s}]$ & \\
\hline k02 & $1[\mathrm{~cm} / \mathrm{s}]$ & $\mathrm{k} 0$ for DMFc+ IT \\
\hline alpha & 0.5 & \\
\hline cMaqi & $10[\mathrm{mmol} / \mathrm{L}]$ & \\
\hline cMorgi & $0[\mathrm{mmol} / \mathrm{L}]$ & \\
\hline cHO2i & $0[\mathrm{mmol} / \mathrm{L}]$ & \\
\hline cDMFci & $5[\mathrm{mmol} / \mathrm{L}]$ & \\
\hline cDMFcaqi & $0[\mathrm{mmol} / \mathrm{L}]$ & \\
\hline cDMFcplusi & $0[\mathrm{mmol} / \mathrm{L}]$ & \\
\hline cDMFcplusaqi & $0[\mathrm{mmol} / \mathrm{L}]$ & \\
\hline cO2i & $1.6[\mathrm{mmol} / \mathrm{L}]$ & \\
\hline cO2aqi & $1.6[\mathrm{mmol} / \mathrm{L}]$ & \\
\hline cOHaqi & $10[\mathrm{mmol} / \mathrm{L}]$ & \\
\hline cFcHi & $0[\mathrm{mmol} / \mathrm{L}]$ & \\
\hline cH2Oi & $131[\mathrm{mmol} / \mathrm{L}]$ & \\
\hline cMOHi & $0[\mathrm{mmol} / \mathrm{L}]$ & \\
\hline
\end{tabular}




\begin{tabular}{|l|l|l|}
\hline Name & Expression & Description \\
\hline cHO2minusi & $0[\mathrm{mmol} / \mathrm{L}]$ & \\
\hline test1 & $\mathrm{R}^{*} \mathrm{~T} / \mathrm{F}$ & \\
\hline n1 & 1 & number of electrons \\
\hline n2 & -1 & charge on Cl- \\
\hline D_DMFc & $7.26 \mathrm{e}-9\left[\mathrm{~m}^{\wedge} 2 / \mathrm{s}\right]$ & \\
\hline D_M & $1.0 \mathrm{e}-5[\mathrm{~cm} \wedge 2 / \mathrm{s}]$ & \\
\hline D_DMFcplus & $7.26 \mathrm{e}-6[\mathrm{~cm} \wedge 2 / \mathrm{s}]$ & \\
\hline D_H2O & $6.53 \mathrm{e}-9\left[\mathrm{~m}^{\wedge} 2 / \mathrm{s}\right]$ & \\
\hline D_O2 & $2.76 \mathrm{e}-5[\mathrm{~cm} \wedge 2 / \mathrm{s}]$ & \\
\hline D_Cl & $1 \mathrm{e}-9\left[\mathrm{~m}^{\wedge} 2 / \mathrm{s}\right]$ & \\
\hline D_Horg & $1 \mathrm{e}-9\left[\mathrm{~m}^{\wedge} 2 / \mathrm{s}\right]$ & \\
\hline D_H2O2 & $1 \mathrm{e}-5[\mathrm{~cm} \wedge 2 / \mathrm{s}]$ & \\
\hline kfo2 & $0[\mathrm{~cm} / \mathrm{s}]$ & \\
\hline kbo2 & $0[\mathrm{~cm} / \mathrm{s}]$ & \\
\hline kcf & $10[\mathrm{~L} / \mathrm{mol} / \mathrm{s}]$ & hydride formation \\
\hline kcb & $1[\mathrm{~L} / \mathrm{mol} / \mathrm{s}]$ & \\
\hline kbFc & $1[\mathrm{~cm} / \mathrm{s}]$ & \\
\hline kchem1 & $10[\mathrm{~L} / \mathrm{mol} / \mathrm{s}]$ & HO2 formation \\
\hline kchem2 & $1 \mathrm{e}+4[\mathrm{~L} / \mathrm{mol} / \mathrm{s}]$ & reaction of DMFcH+ + O2 \\
\hline kchem3 & $1 \mathrm{e}+4[\mathrm{~L} / \mathrm{mol} / \mathrm{s}]$ & \\
\hline kchem4 & $1 \mathrm{e}+4[\mathrm{~L} / \mathrm{mol} / \mathrm{s}]$ & HO2- + M --> H2O2 + MOH \\
\hline kmohf & $1 \mathrm{e}+8[\mathrm{~m} / \mathrm{s}]$ & MOH partitioning \\
\hline kmohb & $1[\mathrm{~m} / \mathrm{s}]$ & \\
\hline kmf & $1 \mathrm{e}+5[1 / \mathrm{s}]$ & complexation in the aqueous phase \\
\hline kmb & $1[\mathrm{~L} / \mathrm{mol} / \mathrm{s}]$ & \\
\hline kcf2 & $5000[\mathrm{~L} / \mathrm{mol} / \mathrm{s}]$ & organic phase MOH formation aqueous phase \\
\hline kcb2 & $1[1 / \mathrm{s}]$ & \\
\hline cOHorgi & $0[\mathrm{~mol} / \mathrm{L}]$ & \\
\hline D_OH & $1 \mathrm{e}-9\left[\mathrm{~m}^{\wedge} 2 / \mathrm{s}\right]$ & OH diffusion coefficient \\
\hline
\end{tabular}




\begin{tabular}{|l|l|l|}
\hline Name & Expression & Description \\
\hline rd & $0.7[\mathrm{~cm}]$ & \\
\hline
\end{tabular}

\subsection{Variables}

\subsubsection{Variables 1}

Selection

\begin{tabular}{|l|l|}
\hline Geometric entity level & Entire model \\
\hline
\end{tabular}

\begin{tabular}{|c|c|c|}
\hline Name & Expression & Description \\
\hline E_swp & $E \mathrm{i}+\left(2^{*}(\mathrm{Ef}-\mathrm{Ei})\right) / \mathrm{pi} * \operatorname{asin}\left(\sin \left(\left(\mathrm{pi}^{*} \mathrm{nu}^{*} \mathrm{t}\right) /\left(2^{*}(\mathrm{Ef}-\mathrm{Ei})\right)\right)\right)$ & \\
\hline E_swp2 & $(E \mathrm{i}-\mathrm{nu} * \mathrm{t}) *(\mathrm{t}<=25)+(\mathrm{Ef}+\mathrm{nu} *(\mathrm{t}-25)) *(\mathrm{t}>25)$ & \\
\hline $\mathrm{kf}$ & k0*exp(-alpha*fara*n1*(E_swp - E0)) & Metal \\
\hline $\mathrm{kb}$ & k0*exp((1 - alpha)*fara*n1*(E_swp - E0)) & \\
\hline E_swp3 & $\mathrm{Ei}$ & \\
\hline kf2 & k02*exp(-alpha*fara*n1*(E_swp - E02)) & DMFc $^{+}$ \\
\hline kb2 & k02*exp((1 - alpha)*fara*n1*(E_swp - E02)) & \\
\hline kf3 & k0*exp(-alpha*fara*n2*(E_swp - E03)) & hydroxyl \\
\hline kb3 & k0*exp((1 - alpha)*fara*n2*(E_swp - E03) $)$ & \\
\hline
\end{tabular}

\subsubsection{Variables 2}

Selection

\begin{tabular}{|l|l|}
\hline Geometric entity level & Entire model \\
\hline
\end{tabular}

\begin{tabular}{|l|l|l|}
\hline Name & Expression & Description \\
\hline Ibar & comp1.intop1(intcpl_source_Ibar) & \\
\hline
\end{tabular}


2 Component 1 (comp1)

\subsection{Definitions}

\subsubsection{Variables}

Variables 3

Selection

\begin{tabular}{|l|l|}
\hline Geometric entity level & Boundary \\
\hline Selection & Boundary 2 \\
\hline
\end{tabular}

\begin{tabular}{|l|l|l|}
\hline Name & Expression & Description \\
\hline intcpl_source_Ibar & $\begin{array}{l}\mathrm{pi}^{*}(\mathrm{rd} \wedge 2) * \mathrm{~F}^{*}(\text { chds.ndflux_Maq - chds.ndflux_OHaq } \\
+ \text { chds.ndflux_Fcplusaq })\end{array}$ & \\
\hline
\end{tabular}

\subsubsection{Component Couplings}

Integration 1

\begin{tabular}{|l|l|}
\hline Coupling type & Integration \\
\hline Operator name & intop1 \\
\hline
\end{tabular}

Source selection

\begin{tabular}{|l|l|}
\hline Geometric entity level & Boundary \\
\hline Selection & Boundary 2 \\
\hline
\end{tabular}




\subsection{Geometry 1}

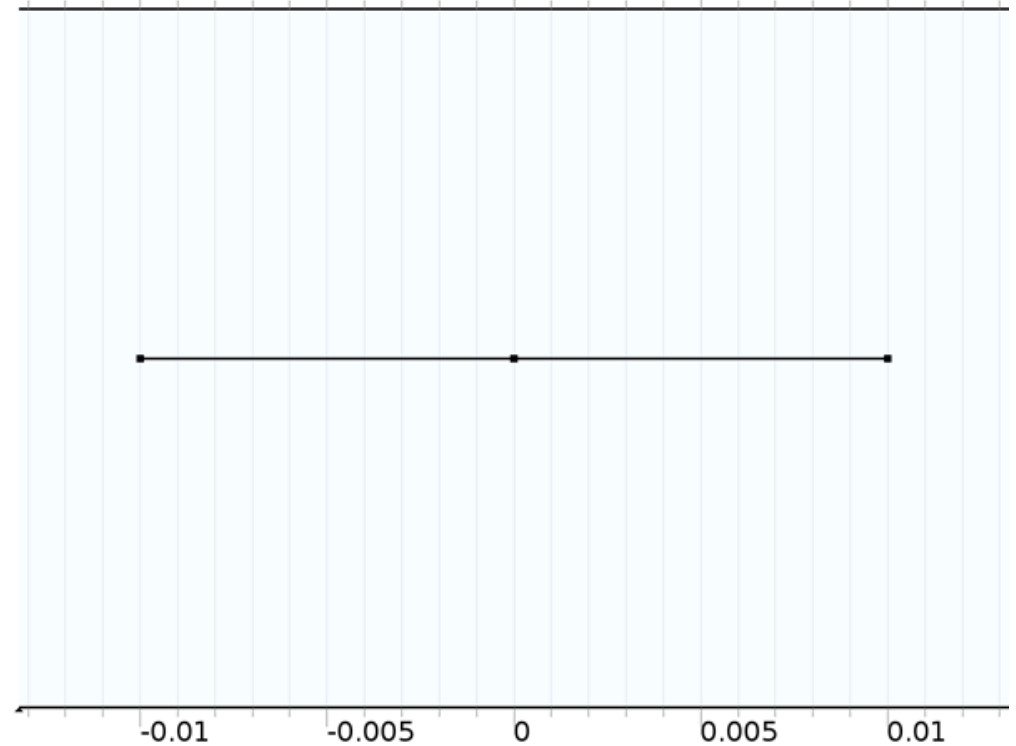

Geometry 1

Units

\begin{tabular}{|l|l|}
\hline Length unit & $\mathrm{m}$ \\
\hline Angular unit & deg \\
\hline
\end{tabular}

Geometry statistics

\begin{tabular}{|l|l|}
\hline Property & Value \\
\hline Space dimension & 1 \\
\hline Number of domains & 2 \\
\hline Number of boundaries & 3 \\
\hline
\end{tabular}

\subsubsection{Interval 1 (i1)}

Selections of resulting entities

\begin{tabular}{|l|l|}
\hline Name & Value \\
\hline Number of intervals & One \\
\hline Left endpoint & -0.01 \\
\hline Right endpoint & 0 \\
\hline
\end{tabular}

2.2.2 Interval 2 (i2)

Selections of resulting entities 


\begin{tabular}{|l|l|}
\hline Name & Value \\
\hline Number of intervals & One \\
\hline Left endpoint & 0 \\
\hline Right endpoint & 0.01 \\
\hline
\end{tabular}

\subsection{Transport of Diluted Species (chds)}

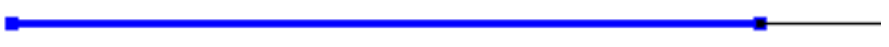

\begin{tabular}{lllllll}
\hline-0.01 & -0.008 & -0.006 & -0.004 & -0.002 & 0
\end{tabular}

Transport of Diluted Species

Selection

\begin{tabular}{|l|l|}
\hline Geometric entity level & Domain \\
\hline Selection & Domain 1 \\
\hline
\end{tabular}

Equations

$$
\begin{aligned}
& \frac{\partial c_{i}}{\partial t}+\nabla \cdot\left(-D_{i} \nabla c_{i}\right)+\mathbf{u} \cdot \nabla c_{i}=R_{i} \\
& \mathbf{N}_{i}=-D_{i} \nabla c_{i}+\mathbf{u} c_{i}
\end{aligned}
$$

Settings

\begin{tabular}{|l|l|}
\hline Description & Value \\
\hline Concentration & Linear \\
\hline Compute boundary fluxes & On \\
\hline Apply smoothing to boundary fluxes & On \\
\hline Value type when using splitting of complex variables & Real \\
\hline Migration in electric field & 0 \\
\hline
\end{tabular}




\begin{tabular}{|l|l|}
\hline Description & Value \\
\hline Convection & 1 \\
\hline Convective term & Non - conservative form \\
\hline Equation residual & Approximate residual \\
\hline Enable space-dependent physics interfaces & 0 \\
\hline Synchronize with COMSOL Multiphysics & \\
\hline
\end{tabular}

Used products

\begin{tabular}{|l|}
\hline COMSOL Multiphysics \\
\hline Chemical Reaction Engineering Module \\
\hline
\end{tabular}

\begin{tabular}{|l|l|l|l|l|}
\hline Name & Expression & Unit & Description & Selection \\
\hline chds.nx & unx & $\begin{array}{l}\text { Normal } \\
\text { vector, x } \\
\text { component }\end{array}$ & Boundary 1 \\
\hline chds.ny & 0 & & $\begin{array}{l}\text { Normal } \\
\text { vector, y } \\
\text { component }\end{array}$ & Boundary 1 \\
\hline chds.nz & 0 & & $\begin{array}{l}\text { Normal } \\
\text { vector, z } \\
\text { component }\end{array}$ & Boundary 1 \\
\hline chds.nx & dnx & $\begin{array}{l}\text { Normal } \\
\text { vector, x } \\
\text { component }\end{array}$ & Boundary 2 \\
\hline chds.ny & 0 & $\begin{array}{l}\text { Normal } \\
\text { vector, y } \\
\text { component }\end{array}$ & Boundary 2 \\
\hline chds.nz & 0 & $\begin{array}{l}\text { Normal } \\
\text { vector, z } \\
\text { component }\end{array}$ & Boundary 2 \\
\hline
\end{tabular}




\begin{tabular}{|c|c|c|c|c|}
\hline Name & \begin{tabular}{|l|} 
Expression \\
\end{tabular} & Unit & Description & Selection \\
\hline chds.nxmesh & root.unxmesh & & $\begin{array}{l}\text { Normal } \\
\text { vector } \\
\text { (mesh), x } \\
\text { component }\end{array}$ & Boundary 1 \\
\hline chds.nymesh & 0 & & $\begin{array}{l}\text { Normal } \\
\text { vector } \\
\text { (mesh), y } \\
\text { component }\end{array}$ & Boundary 1 \\
\hline chds.nzmesh & 0 & & $\begin{array}{l}\text { Normal } \\
\text { vector } \\
\text { (mesh), z } \\
\text { component }\end{array}$ & Boundary 1 \\
\hline chds.nxmesh & root.dnxmesh & & $\begin{array}{l}\text { Normal } \\
\text { vector } \\
\text { (mesh), x } \\
\text { component }\end{array}$ & Boundary 2 \\
\hline chds.nymesh & 0 & & $\begin{array}{l}\text { Normal } \\
\text { vector } \\
\text { (mesh), y } \\
\text { component }\end{array}$ & Boundary 2 \\
\hline chds.nzmesh & 0 & & $\begin{array}{l}\text { Normal } \\
\text { vector } \\
\text { (mesh), z } \\
\text { component }\end{array}$ & Boundary 2 \\
\hline chds.R_Maq & 0 & $\mathrm{~mol} /\left(\mathrm{m}^{\wedge} 3^{*} \mathrm{~s}\right)$ & $\begin{array}{l}\text { Total rate } \\
\text { expression }\end{array}$ & Domain 1 \\
\hline chds.R_OHaq & 0 & $\mathrm{~mol} /\left(\mathrm{m}^{\wedge} 3^{*} \mathrm{~s}\right)$ & $\begin{array}{l}\text { Total rate } \\
\text { expression }\end{array}$ & Domain 1 \\
\hline chds.R_Fcplusaq & 0 & $\mathrm{~mol} /\left(\mathrm{m}^{\wedge} 3^{*} \mathrm{~s}\right)$ & $\begin{array}{l}\text { Total rate } \\
\text { expression }\end{array}$ & Domain 1 \\
\hline
\end{tabular}




\begin{tabular}{|c|c|c|c|c|}
\hline Name & Expression & Unit & Description & Selection \\
\hline chds.R_MOH & 0 & $\mathrm{~mol} /\left(\mathrm{m}^{\wedge} 3^{*} \mathrm{~s}\right)$ & $\begin{array}{l}\text { Total rate } \\
\text { expression }\end{array}$ & Domain 1 \\
\hline domflux.Maqx & chds.dfluxx_Maq & $\mathrm{mol} /\left(\mathrm{m}^{\wedge} 2 * \mathrm{~s}\right)$ & Domain flux & Domain 1 \\
\hline domflux.OHaqx & chds.dfluxx_OHaq & $\mathrm{mol} /\left(\mathrm{m}^{\wedge} 2^{*} \mathrm{~s}\right)$ & Domain flux & Domain 1 \\
\hline domflux.Fcplusaqx & chds.dfluxx_Fcplusaq & $\mathrm{mol} /\left(\mathrm{m}^{\wedge} 2 * \mathrm{~s}\right)$ & Domain flux & Domain 1 \\
\hline domflux.MOHx & chds.dfluxx_MOH & $\mathrm{mol} /\left(\mathrm{m}^{\wedge} 2^{*} \mathrm{~s}\right)$ & Domain flux & Domain 1 \\
\hline chds.bndFlux_Maq & -uflux_spatial(Maq) & $\mathrm{mol} /\left(\mathrm{m}^{\wedge} 2^{*} \mathrm{~s}\right)$ & $\begin{array}{l}\text { Boundary } \\
\text { flux }\end{array}$ & Boundary 1 \\
\hline chds.bndFlux_Maq & -dflux_spatial(Maq) & $\mathrm{mol} /\left(\mathrm{m}^{\wedge} 2 * \mathrm{~s}\right)$ & $\begin{array}{l}\text { Boundary } \\
\text { flux }\end{array}$ & Boundary 2 \\
\hline chds.ntflux_Maq & $\begin{array}{l}\text { chds.bndFlux_Maq+c } \\
\text { hds.cfluxx_Maq*chds. } \\
\text { nx+chds.cfluxy_Maq* } \\
\text { chds.ny+chds.cfluxz_ } \\
\text { Maq*chds.nz }\end{array}$ & $\mathrm{mol} /\left(\mathrm{m}^{\wedge} 2 * \mathrm{~s}\right)$ & $\begin{array}{l}\text { Normal total } \\
\text { flux }\end{array}$ & $\begin{array}{l}\text { Boundaries } \\
1-2\end{array}$ \\
\hline chds.ndflux_Maq & chds.bndFlux_Maq & $\mathrm{mol} /\left(\mathrm{m}^{\wedge} 2^{*} \mathrm{~s}\right)$ & $\begin{array}{l}\text { Normal } \\
\text { diffusive flux }\end{array}$ & $\begin{array}{l}\text { Boundaries } \\
1-2\end{array}$ \\
\hline chds.ncflux_Maq & 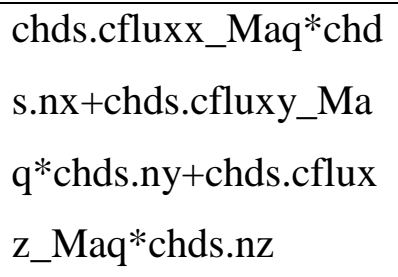 & $\mathrm{mol} /\left(\mathrm{m}^{\wedge} 2 * \mathrm{~s}\right)$ & $\begin{array}{l}\text { Normal } \\
\text { convective } \\
\text { flux }\end{array}$ & $\begin{array}{l}\text { Boundaries } \\
1-2\end{array}$ \\
\hline chds.bndFlux_OHaq & -uflux_spatial(OHaq) & $\mathrm{mol} /\left(\mathrm{m}^{\wedge} 2^{*} \mathrm{~s}\right)$ & $\begin{array}{l}\text { Boundary } \\
\text { flux }\end{array}$ & Boundary 1 \\
\hline chds.bndFlux_OHaq & -dflux_spatial(OHaq) & $\mathrm{mol} /\left(\mathrm{m}^{\wedge} 2 * \mathrm{~s}\right)$ & $\begin{array}{l}\text { Boundary } \\
\text { flux }\end{array}$ & Boundary 2 \\
\hline chds.ntflux_OHaq & $\begin{array}{l}\text { chds.bndFlux_OHaq+ } \\
\text { chds.cfluxx_OHaq*ch } \\
\text { ds.nx+chds.cfluxy_O }\end{array}$ & $\mathrm{mol} /\left(\mathrm{m}^{\wedge} 2 * \mathrm{~s}\right)$ & $\begin{array}{l}\text { Normal total } \\
\text { flux }\end{array}$ & $\begin{array}{l}\text { Boundaries } \\
1-2\end{array}$ \\
\hline
\end{tabular}




\begin{tabular}{|c|c|c|c|c|}
\hline Name & Expression & Unit & Description & Selection \\
\hline & $\begin{array}{l}\text { Haq*chds.ny+chds.cfl } \\
\text { uxz_OHaq*chds.nz }\end{array}$ & & & \\
\hline chds.ndflux_OHaq & chds.bndFlux_OHaq & $\mathrm{mol} /\left(\mathrm{m}^{\wedge} 2 * \mathrm{~s}\right)$ & $\begin{array}{l}\text { Normal } \\
\text { diffusive flux }\end{array}$ & $\begin{array}{l}\text { Boundaries } \\
1-2\end{array}$ \\
\hline chds.ncflux_OHaq & $\begin{array}{l}\text { chds.cfluxx_OHaq*ch } \\
\text { ds.nx+chds.cfluxy_O } \\
\text { Haq*chds.ny+chds.cfl } \\
\text { uxz_OHaq*chds.nz }\end{array}$ & $\mathrm{mol} /\left(\mathrm{m}^{\wedge} 2 * \mathrm{~s}\right)$ & $\begin{array}{l}\text { Normal } \\
\text { convective } \\
\text { flux }\end{array}$ & $\begin{array}{l}\text { Boundaries } \\
1-2\end{array}$ \\
\hline $\begin{array}{l}\text { chds.bndFlux_Fcplusa } \\
\text { q }\end{array}$ & $\begin{array}{l}\text { - } \\
\text { uflux_spatial(Fcplusa } \\
\text { q) }\end{array}$ & $\mathrm{mol} /\left(\mathrm{m}^{\wedge} 2^{*} \mathrm{~s}\right)$ & $\begin{array}{l}\text { Boundary } \\
\text { flux }\end{array}$ & Boundary 1 \\
\hline $\begin{array}{l}\text { chds.bndFlux_Fcplusa } \\
\text { q }\end{array}$ & $\begin{array}{l}\text { - } \\
\text { dflux_spatial(Fcplusa } \\
\text { q) }\end{array}$ & $\mathrm{mol} /\left(\mathrm{m}^{\wedge} 2 * \mathrm{~s}\right)$ & $\begin{array}{l}\text { Boundary } \\
\text { flux }\end{array}$ & Boundary 2 \\
\hline chds.ntflux_Fcplusaq & $\begin{array}{l}\text { chds.bndFlux_Fcplusa } \\
\text { q+chds.cfluxx_Fcplus } \\
\text { aq*chds.nx+chds.cflu } \\
\text { xy_Fcplusaq*chds.ny } \\
\text { +chds.cfluxz_Fcplusa } \\
\text { q*chds.nz }\end{array}$ & $\mathrm{mol} /\left(\mathrm{m}^{\wedge} 2 * \mathrm{~s}\right)$ & $\begin{array}{l}\text { Normal total } \\
\text { flux }\end{array}$ & $\begin{array}{l}\text { Boundaries } \\
1-2\end{array}$ \\
\hline chds.ndflux_Fcplusaq & $\begin{array}{l}\text { chds.bndFlux_Fcplusa } \\
\text { q }\end{array}$ & $\mathrm{mol} /\left(\mathrm{m}^{\wedge} 2 * \mathrm{~s}\right)$ & $\begin{array}{l}\text { Normal } \\
\text { diffusive flux }\end{array}$ & $\begin{array}{l}\text { Boundaries } \\
1-2\end{array}$ \\
\hline chds.ncflux_Fcplusaq & $\begin{array}{l}\text { chds.cfluxx_Fcplusaq } \\
\text { *chds.nx+chds.cfluxy } \\
\text { _Fcplusaq*chds.ny+c } \\
\text { hds.cfluxz_Fcplusaq* } \\
\text { chds.nz }\end{array}$ & $\mathrm{mol} /\left(\mathrm{m}^{\wedge} 2 * \mathrm{~s}\right)$ & $\begin{array}{l}\text { Normal } \\
\text { convective } \\
\text { flux }\end{array}$ & $\begin{array}{l}\text { Boundaries } \\
1-2\end{array}$ \\
\hline chds.bndFlux_MOH & -uflux_spatial(MOH) & $\mathrm{mol} /\left(\mathrm{m}^{\wedge} 2 * \mathrm{~s}\right)$ & $\begin{array}{l}\text { Boundary } \\
\text { flux }\end{array}$ & Boundary 1 \\
\hline
\end{tabular}




\begin{tabular}{|c|c|c|c|c|}
\hline Name & Expression & Unit & Description & Selection \\
\hline chds.bndFlux_MOH & -dflux_spatial(MOH) & $\mathrm{mol} /\left(\mathrm{m}^{\wedge} 2 * \mathrm{~s}\right)$ & $\begin{array}{l}\text { Boundary } \\
\text { flux }\end{array}$ & Boundary 2 \\
\hline chds.ntflux_MOH & $\begin{array}{l}\text { chds.bndFlux_MOH+ } \\
\text { chds.cfluxx_MOH*ch } \\
\text { ds.nx+chds.cfluxy_M } \\
\text { OH*chds.ny+chds.cfl } \\
\text { uxz_MOH*chds.nz }\end{array}$ & $\mathrm{mol} /\left(\mathrm{m}^{\wedge} 2 * \mathrm{~s}\right)$ & $\begin{array}{l}\text { Normal total } \\
\text { flux }\end{array}$ & $\begin{array}{l}\text { Boundaries } \\
1-2\end{array}$ \\
\hline chds.ndflux_MOH & chds.bndFlux_MOH & $\mathrm{mol} /\left(\mathrm{m}^{\wedge} 2^{*} \mathrm{~s}\right)$ & $\begin{array}{l}\text { Normal } \\
\text { diffusive flux }\end{array}$ & $\begin{array}{l}\text { Boundaries } \\
1-2\end{array}$ \\
\hline chds.ncflux_MOH & $\begin{array}{l}\text { chds.cfluxx_MOH*ch } \\
\text { ds.nx+chds.cfluxy_M } \\
\text { OH*chds.ny+chds.cfl } \\
\text { uxz_MOH*chds.nz }\end{array}$ & $\mathrm{mol} /\left(\mathrm{m}^{\wedge} 2 * \mathrm{~s}\right)$ & $\begin{array}{l}\text { Normal } \\
\text { convective } \\
\text { flux }\end{array}$ & $\begin{array}{l}\text { Boundaries } \\
1-2\end{array}$ \\
\hline
\end{tabular}

\subsubsection{Convection and Diffusion 1}

$\begin{array}{llllll}-0.01 & -0.008 & -0.006 & -0.004 & -0.002 & 0\end{array}$

Convection and Diffusion 1

Selection

\begin{tabular}{|l|l|}
\hline Geometric entity level & Domain \\
\hline Selection & Domain 1 \\
\hline
\end{tabular}


Equations

$$
\begin{aligned}
& \frac{\partial c_{i}}{\partial t}+\nabla \cdot\left(-D_{i} \nabla c_{i}\right)+\mathbf{u} \cdot \nabla c_{i}=R_{i} \\
& \mathbf{N}_{i}=-D_{i} \nabla c_{i}+\mathbf{u} c_{i}
\end{aligned}
$$

\section{Settings}

\begin{tabular}{|c|c|}
\hline Description & Value \\
\hline Velocity field & User defined \\
\hline Velocity field & $\{0,0,0\}$ \\
\hline Electric potential & User defined \\
\hline Electric potential & 0 \\
\hline Diffusion coefficient & User defined \\
\hline Diffusion coefficient & $\left\{\left\{\mathrm{D} \_\mathrm{M}, 0,0\right\},\left\{0, \mathrm{D} \_\mathrm{M}, 0\right\},\left\{0,0, \mathrm{D} \_\mathrm{M}\right\}\right\}$ \\
\hline Diffusion coefficient & User defined \\
\hline Diffusion coefficient & $\left\{\left\{\mathrm{D} \_\mathrm{Cl}, 0,0\right\},\left\{0, \mathrm{D} \_\mathrm{Cl}, 0\right\},\left\{0,0, \mathrm{D} \_\mathrm{Cl}\right\}\right\}$ \\
\hline Diffusion coefficient & User defined \\
\hline Diffusion coefficient & $\{\{$ D_DMFcplus, 0,0$\},\{0$, D_DMFcplus, 0$\},\{0,0$, D_DMFcplus $\}\}$ \\
\hline Diffusion coefficient & User defined \\
\hline Diffusion coefficient & $\left\{\left\{\mathrm{D} \_\mathrm{M}, 0,0\right\},\left\{0, \mathrm{D} \_\mathrm{M}, 0\right\},\left\{0,0, \mathrm{D} \_\mathrm{M}\right\}\right\}$ \\
\hline Bulk material & None \\
\hline
\end{tabular}

Settings

\section{Used products}

COMSOL Multiphysics

\section{Variables}

\begin{tabular}{|l|l|l|l|l|}
\hline Name & Expression & Unit & Description & Selection \\
\hline chds.cbf_Maq & 0 & $\mathrm{~mol} /\left(\mathrm{m}^{\wedge} 2^{*} \mathrm{~s}\right)$ & $\begin{array}{l}\text { Convective } \\
\text { boundary flux }\end{array}$ & $\begin{array}{l}\text { Boundaries } \\
1-2\end{array}$ \\
\hline chds.cbf_OHaq & 0 & $\mathrm{~mol} /\left(\mathrm{m}^{\wedge} 2^{*} \mathrm{~s}\right)$ & $\begin{array}{l}\text { Convective } \\
\text { boundary flux }\end{array}$ & $\begin{array}{l}\text { Boundaries } \\
1-2\end{array}$ \\
\hline $\begin{array}{l}\text { chds.cbf_Fcplu } \\
\text { saq }\end{array}$ & 0 & $\mathrm{~mol} /\left(\mathrm{m}^{\wedge} 2^{*} \mathrm{~s}\right)$ & $\begin{array}{l}\text { Convective } \\
\text { boundary flux }\end{array}$ & $\begin{array}{l}\text { Boundaries } \\
1-2\end{array}$ \\
\hline
\end{tabular}




\begin{tabular}{|c|c|c|c|c|}
\hline Name & Expression & Unit & Description & Selection \\
\hline chds.cbf_MOH & 0 & $\mathrm{~mol} /\left(\mathrm{m}^{\wedge} 2 * \mathrm{~s}\right)$ & $\begin{array}{l}\text { Convective } \\
\text { boundary flux }\end{array}$ & $\begin{array}{l}\text { Boundaries } \\
1-2\end{array}$ \\
\hline chds.Dxx_Maq & D_M & $\mathrm{m}^{\wedge} 2 / \mathrm{s}$ & $\begin{array}{l}\text { Diffusion } \\
\text { coefficient, } \mathrm{xx} \\
\text { component }\end{array}$ & Domain 1 \\
\hline chds.Dyx_Maq & 0 & $m^{\wedge} 2 / s$ & $\begin{array}{l}\text { Diffusion } \\
\text { coefficient, yx } \\
\text { component }\end{array}$ & Domain 1 \\
\hline chds.Dzx_Maq & 0 & $\mathrm{~m}^{\wedge} 2 / \mathrm{s}$ & $\begin{array}{l}\text { Diffusion } \\
\text { coefficient, zx } \\
\text { component }\end{array}$ & Domain 1 \\
\hline chds.Dxy_Maq & 0 & $\mathrm{~m}^{\wedge} 2 / \mathrm{s}$ & $\begin{array}{l}\text { Diffusion } \\
\text { coefficient, xy } \\
\text { component }\end{array}$ & Domain 1 \\
\hline chds.Dyy_Maq & D_M & $\mathrm{m}^{\wedge} 2 / \mathrm{s}$ & $\begin{array}{l}\text { Diffusion } \\
\text { coefficient, yy } \\
\text { component }\end{array}$ & Domain 1 \\
\hline chds.Dzy_Maq & 0 & $\mathrm{~m}^{\wedge} 2 / \mathrm{s}$ & $\begin{array}{l}\text { Diffusion } \\
\text { coefficient, zy } \\
\text { component }\end{array}$ & Domain 1 \\
\hline chds.Dxz_Maq & 0 & $\mathrm{~m}^{\wedge} 2 / \mathrm{s}$ & $\begin{array}{l}\text { Diffusion } \\
\text { coefficient, } x z \\
\text { component }\end{array}$ & Domain 1 \\
\hline chds.Dyz_Maq & 0 & $\mathrm{~m}^{\wedge} 2 / \mathrm{s}$ & $\begin{array}{l}\text { Diffusion } \\
\text { coefficient, yz } \\
\text { component }\end{array}$ & Domain 1 \\
\hline chds.Dzz_Maq & D_M & $\mathrm{m}^{\wedge} 2 / \mathrm{s}$ & $\begin{array}{l}\text { Diffusion } \\
\text { coefficient, } \quad \mathrm{zz} \\
\text { component }\end{array}$ & Domain 1 \\
\hline
\end{tabular}




\begin{tabular}{|c|c|c|c|c|}
\hline Name & Expression & Unit & Description & Selection \\
\hline chds.Dav_Maq & chds.Dxx_Maq & $\mathrm{m}^{\wedge} 2 / \mathrm{s}$ & $\begin{array}{l}\text { Average } \\
\text { diffusion } \\
\text { coefficient }\end{array}$ & Domain 1 \\
\hline $\begin{array}{l}\text { chds.tfluxx_Ma } \\
\text { q }\end{array}$ & $\begin{array}{l}\text { - } \\
\text { chds.Dxx_Maq*Maqx+chd } \\
\text { s.cfluxx_Maq }\end{array}$ & $\mathrm{mol} /\left(\mathrm{m}^{\wedge} 2^{*} \mathrm{~s}\right)$ & $\begin{array}{l}\text { Total flux, } \mathrm{x} \\
\text { component }\end{array}$ & Domain 1 \\
\hline $\begin{array}{l}\text { chds.tfluxy_Ma } \\
\text { q }\end{array}$ & $\begin{array}{l}\text { - } \\
\text { chds.Dyx_Maq*Maqx+chd } \\
\text { s.cfluxy_Maq }\end{array}$ & $\mathrm{mol} /\left(\mathrm{m}^{\wedge} 2^{*} \mathrm{~s}\right)$ & $\begin{array}{l}\text { Total flux, y } \\
\text { component }\end{array}$ & Domain 1 \\
\hline $\begin{array}{l}\text { chds.tfluxz_Ma } \\
\text { q }\end{array}$ & $\begin{array}{l}\text { - } \\
\text { chds.Dzx_Maq*Maqx+chd } \\
\text { s.cfluxz_Maq }\end{array}$ & $\mathrm{mol} /\left(\mathrm{m}^{\wedge} 2^{*} \mathrm{~s}\right)$ & $\begin{array}{l}\text { Total flux, } \mathrm{z} \\
\text { component }\end{array}$ & Domain 1 \\
\hline $\begin{array}{l}\text { chds.dfluxx_M } \\
\text { aq }\end{array}$ & -chds.Dxx_Maq*Maqx & $\mathrm{mol} /\left(\mathrm{m}^{\wedge} 2^{*} \mathrm{~s}\right)$ & $\begin{array}{l}\text { Diffusive flux, } \\
\text { x component }\end{array}$ & Domain 1 \\
\hline $\begin{array}{l}\text { chds.dfluxy_M } \\
\text { aq }\end{array}$ & -chds.Dyx_Maq*Maqx & $\mathrm{mol} /\left(\mathrm{m}^{\wedge} 2^{*} \mathrm{~s}\right)$ & $\begin{array}{l}\text { Diffusive flux, } \\
\text { y component }\end{array}$ & Domain 1 \\
\hline $\begin{array}{l}\text { chds.dfluxz_M } \\
\text { aq }\end{array}$ & -chds.Dzx_Maq*Maqx & $\mathrm{mol} /\left(\mathrm{m}^{\wedge} 2^{*} \mathrm{~s}\right)$ & $\begin{array}{l}\text { Diffusive flux, } \\
\text { z component }\end{array}$ & Domain 1 \\
\hline $\begin{array}{l}\text { chds.gradx_Ma } \\
\text { q }\end{array}$ & Maqx & $\mathrm{mol} / \mathrm{m}^{\wedge} 4$ & $\begin{array}{l}\text { Concentration } \\
\text { gradient, } \quad \mathrm{X} \\
\text { component }\end{array}$ & Domain 1 \\
\hline $\begin{array}{l}\text { chds.grady_Ma } \\
\text { q }\end{array}$ & 0 & $\mathrm{~mol} / \mathrm{m}^{\wedge} 4$ & $\begin{array}{l}\text { Concentration } \\
\text { gradient, y } \\
\text { component }\end{array}$ & Domain 1 \\
\hline $\begin{array}{l}\text { chds.gradz_Ma } \\
\text { q }\end{array}$ & 0 & $\mathrm{~mol} / \mathrm{m}^{\wedge} 4$ & $\begin{array}{l}\text { Concentration } \\
\text { gradient, } \quad \mathrm{Z} \\
\text { component }\end{array}$ & Domain 1 \\
\hline
\end{tabular}




\begin{tabular}{|c|c|c|c|c|}
\hline Name & Expression & Unit & Description & Selection \\
\hline $\begin{array}{l}\text { chds.dfluxMag } \\
\text { _Maq }\end{array}$ & $\begin{array}{l}\text { sqrt(chds.dfluxx_Maq^2+c } \\
\text { hds.dfluxy_Maq^2+chds.df } \\
\text { luxz_Maq^2) }\end{array}$ & $\mathrm{mol} /\left(\mathrm{m}^{\wedge} 2 * \mathrm{~s}\right)$ & $\begin{array}{l}\text { Diffusive flux } \\
\text { magnitude }\end{array}$ & Domain 1 \\
\hline $\begin{array}{l}\text { chds.tfluxMag_ } \\
\text { Maq }\end{array}$ & $\begin{array}{l}\text { sqrt(chds.tfluxx_Maq^2+ch } \\
\text { ds.tfluxy_Maq^2+chds.tflu } \\
\text { xz_Maq^2) }\end{array}$ & $\mathrm{mol} /\left(\mathrm{m}^{\wedge} 2 * \mathrm{~s}\right)$ & $\begin{array}{l}\text { Total flux } \\
\text { magnitude }\end{array}$ & Domain 1 \\
\hline $\begin{array}{l}\text { chds.Dxx_OHa } \\
\text { q }\end{array}$ & D_Cl & $\mathrm{m}^{\wedge} 2 / \mathrm{s}$ & $\begin{array}{l}\text { Diffusion } \\
\text { coefficient, } \mathrm{xx} \\
\text { component }\end{array}$ & Domain 1 \\
\hline $\begin{array}{l}\text { chds.Dyx_OHa } \\
\text { q }\end{array}$ & 0 & $\mathrm{~m}^{\wedge} 2 / \mathrm{s}$ & $\begin{array}{l}\text { Diffusion } \\
\text { coefficient, yx } \\
\text { component }\end{array}$ & Domain 1 \\
\hline $\begin{array}{l}\text { chds.Dzx_OHa } \\
\text { q }\end{array}$ & 0 & $\mathrm{~m}^{\wedge} 2 / \mathrm{s}$ & $\begin{array}{l}\text { Diffusion } \\
\text { coefficient, } \mathrm{zx} \\
\text { component }\end{array}$ & Domain 1 \\
\hline $\begin{array}{l}\text { chds.Dxy_OHa } \\
\text { q }\end{array}$ & 0 & $\mathrm{~m}^{\wedge} 2 / \mathrm{s}$ & $\begin{array}{l}\text { Diffusion } \\
\text { coefficient, } x y \\
\text { component }\end{array}$ & Domain 1 \\
\hline $\begin{array}{l}\text { chds.Dyy_OHa } \\
\text { q }\end{array}$ & D_Cl & $\mathrm{m}^{\wedge} 2 / \mathrm{s}$ & $\begin{array}{l}\text { Diffusion } \\
\text { coefficient, yy } \\
\text { component }\end{array}$ & Domain 1 \\
\hline $\begin{array}{l}\text { chds.Dzy_OHa } \\
\text { q }\end{array}$ & 0 & $\mathrm{~m}^{\wedge} 2 / \mathrm{s}$ & $\begin{array}{l}\text { Diffusion } \\
\text { coefficient, zy } \\
\text { component }\end{array}$ & Domain 1 \\
\hline $\begin{array}{l}\text { chds.Dxz_OHa } \\
\text { q }\end{array}$ & 0 & $\mathrm{~m}^{\wedge} 2 / \mathrm{s}$ & $\begin{array}{l}\text { Diffusion } \\
\text { coefficient, } \mathrm{xz} \\
\text { component }\end{array}$ & Domain 1 \\
\hline $\begin{array}{l}\text { chds.Dyz_OHa } \\
\text { q }\end{array}$ & 0 & $\mathrm{~m}^{\wedge} 2 / \mathrm{s}$ & $\begin{array}{l}\text { Diffusion } \\
\text { coefficient, yz } \\
\text { component }\end{array}$ & Domain 1 \\
\hline
\end{tabular}




\begin{tabular}{|c|c|c|c|c|}
\hline Name & Expression & Unit & Description & Selection \\
\hline $\begin{array}{l}\text { chds.Dzz_OHa } \\
\text { q }\end{array}$ & D_Cl & $\mathrm{m}^{\wedge} 2 / \mathrm{s}$ & $\begin{array}{l}\text { Diffusion } \\
\text { coefficient, } \quad \mathrm{zz} \\
\text { component }\end{array}$ & Domain 1 \\
\hline $\begin{array}{l}\text { chds.Dav_OHa } \\
\text { q }\end{array}$ & chds.Dxx_OHaq & $\mathrm{m}^{\wedge} 2 / \mathrm{s}$ & $\begin{array}{l}\text { Average } \\
\text { diffusion } \\
\text { coefficient }\end{array}$ & Domain 1 \\
\hline $\begin{array}{l}\text { chds.tfluxx_O } \\
\text { Haq }\end{array}$ & $\begin{array}{l}\text { - } \\
\text { chds.Dxx_OHaq*OHaqx+c } \\
\text { hds.cfluxx_OHaq }\end{array}$ & $\mathrm{mol} /\left(\mathrm{m}^{\wedge} 2^{*} \mathrm{~s}\right)$ & $\begin{array}{l}\text { Total flux, } \mathrm{x} \\
\text { component }\end{array}$ & Domain 1 \\
\hline $\begin{array}{l}\text { chds.tfluxy_O } \\
\text { Haq }\end{array}$ & $\begin{array}{l}\text { - } \\
\text { chds.Dyx_OHaq*OHaqx+c } \\
\text { hds.cfluxy_OHaq }\end{array}$ & $\mathrm{mol} /\left(\mathrm{m}^{\wedge} 2^{*} \mathrm{~s}\right)$ & $\begin{array}{l}\text { Total flux, y } \\
\text { component }\end{array}$ & Domain 1 \\
\hline $\begin{array}{l}\text { chds.tfluxz_O } \\
\text { Haq }\end{array}$ & $\begin{array}{l}\text { - } \\
\text { chds.Dzx_OHaq*OHaqx+c } \\
\text { hds.cfluxz_OHaq }\end{array}$ & $\mathrm{mol} /\left(\mathrm{m}^{\wedge} 2^{*} \mathrm{~s}\right)$ & $\begin{array}{l}\text { Total flux, } \mathrm{z} \\
\text { component }\end{array}$ & Domain 1 \\
\hline $\begin{array}{l}\text { chds.dfluxx_O } \\
\text { Haq }\end{array}$ & -chds.Dxx_OHaq*OHaqx & $\mathrm{mol} /\left(\mathrm{m}^{\wedge} 2^{*} \mathrm{~s}\right)$ & $\begin{array}{l}\text { Diffusive flux, } \\
\text { x component }\end{array}$ & Domain 1 \\
\hline $\begin{array}{l}\text { chds.dfluxy_O } \\
\text { Haq }\end{array}$ & -chds.Dyx_OHaq*OHaqx & $\mathrm{mol} /\left(\mathrm{m}^{\wedge} 2^{*} \mathrm{~s}\right)$ & $\begin{array}{l}\text { Diffusive flux, } \\
\text { y component }\end{array}$ & Domain 1 \\
\hline $\begin{array}{l}\text { chds.dfluxz_O } \\
\text { Haq }\end{array}$ & -chds.Dzx_OHaq*OHaqx & $\mathrm{mol} /\left(\mathrm{m}^{\wedge} 2^{*} \mathrm{~s}\right)$ & $\begin{array}{l}\text { Diffusive flux, } \\
\text { z component }\end{array}$ & Domain 1 \\
\hline $\begin{array}{l}\text { chds.gradx_OH } \\
\text { aq }\end{array}$ & OHaqx & $\mathrm{mol} / \mathrm{m}^{\wedge} 4$ & $\begin{array}{l}\text { Concentration } \\
\text { gradient, } \quad \mathrm{x} \\
\text { component }\end{array}$ & Domain 1 \\
\hline $\begin{array}{l}\text { chds.grady_OH } \\
\text { aq }\end{array}$ & 0 & $\mathrm{~mol} / \mathrm{m}^{\wedge} 4$ & $\begin{array}{l}\text { Concentration } \\
\text { gradient, y } \\
\text { component }\end{array}$ & Domain 1 \\
\hline
\end{tabular}




\begin{tabular}{|c|c|c|c|c|}
\hline Name & Expression & Unit & Description & Selection \\
\hline $\begin{array}{l}\text { chds.gradz_OH } \\
\text { aq }\end{array}$ & 0 & $\mathrm{~mol} / \mathrm{m}^{\wedge} 4$ & $\begin{array}{l}\text { Concentration } \\
\text { gradient, } \quad \mathrm{Z} \\
\text { component }\end{array}$ & Domain 1 \\
\hline $\begin{array}{l}\text { chds.dfluxMag } \\
\text { _OHaq }\end{array}$ & $\begin{array}{l}\text { sqrt(chds.dfluxx_OHaq^2+ } \\
\text { chds.dfluxy_OHaq^2+chds } \\
\text {.dfluxz_OHaq^2) }\end{array}$ & $\mathrm{mol} /\left(\mathrm{m}^{\wedge} 2^{*} \mathrm{~s}\right)$ & $\begin{array}{l}\text { Diffusive flux } \\
\text { magnitude }\end{array}$ & Domain 1 \\
\hline $\begin{array}{l}\text { chds.tfluxMag_ } \\
\text { OHaq }\end{array}$ & $\begin{array}{l}\text { sqrt(chds.tfluxx_OHaq^2+ } \\
\text { chds.tfluxy_OHaq^2+chds. } \\
\text { tfluxz_OHaq^2) }\end{array}$ & $\mathrm{mol} /\left(\mathrm{m}^{\wedge} 2^{*} \mathrm{~s}\right)$ & $\begin{array}{ll}\text { Total flux } \\
\text { magnitude }\end{array}$ & Domain 1 \\
\hline $\begin{array}{l}\text { chds.Dxx_Fcpl } \\
\text { usaq }\end{array}$ & D_DMFcplus & $\mathrm{m}^{\wedge} 2 / \mathrm{s}$ & $\begin{array}{l}\text { Diffusion } \\
\text { coefficient, } \mathrm{xx} \\
\text { component }\end{array}$ & Domain 1 \\
\hline $\begin{array}{l}\text { chds.Dyx_Fcpl } \\
\text { usaq }\end{array}$ & 0 & $\mathrm{~m}^{\wedge} 2 / \mathrm{s}$ & $\begin{array}{l}\text { Diffusion } \\
\text { coefficient, yx } \\
\text { component }\end{array}$ & Domain 1 \\
\hline $\begin{array}{l}\text { chds.Dzx_Fcpl } \\
\text { usaq }\end{array}$ & 0 & $\mathrm{~m}^{\wedge} 2 / \mathrm{s}$ & $\begin{array}{l}\text { Diffusion } \\
\text { coefficient, zx } \\
\text { component }\end{array}$ & Domain 1 \\
\hline $\begin{array}{l}\text { chds.Dxy_Fcpl } \\
\text { usaq }\end{array}$ & 0 & $\mathrm{~m}^{\wedge} 2 / \mathrm{s}$ & $\begin{array}{l}\text { Diffusion } \\
\text { coefficient, } \quad x y \\
\text { component }\end{array}$ & Domain 1 \\
\hline $\begin{array}{l}\text { chds.Dyy_Fcpl } \\
\text { usaq }\end{array}$ & D_DMFcplus & $\mathrm{m}^{\wedge} 2 / \mathrm{s}$ & $\begin{array}{l}\text { Diffusion } \\
\text { coefficient, yy } \\
\text { component }\end{array}$ & Domain 1 \\
\hline $\begin{array}{l}\text { chds.Dzy_Fcpl } \\
\text { usaq }\end{array}$ & 0 & $\mathrm{~m}^{\wedge} 2 / \mathrm{s}$ & $\begin{array}{l}\text { Diffusion } \\
\text { coefficient, zy } \\
\text { component }\end{array}$ & Domain 1 \\
\hline $\begin{array}{l}\text { chds.Dxz_Fcpl } \\
\text { usaq }\end{array}$ & 0 & $\mathrm{~m}^{\wedge} 2 / \mathrm{s}$ & $\begin{array}{l}\text { Diffusion } \\
\text { coefficient, } \mathrm{xz} \\
\text { component }\end{array}$ & Domain 1 \\
\hline
\end{tabular}




\begin{tabular}{|c|c|c|c|c|}
\hline Name & Expression & Unit & Description & Selection \\
\hline $\begin{array}{l}\text { chds.Dyz_Fcpl } \\
\text { usaq }\end{array}$ & 0 & $\mathrm{~m}^{\wedge} 2 / \mathrm{s}$ & $\begin{array}{l}\text { Diffusion } \\
\text { coefficient, yz } \\
\text { component }\end{array}$ & Domain 1 \\
\hline $\begin{array}{l}\text { chds.Dzz_Fcpl } \\
\text { usaq }\end{array}$ & D_DMFcplus & $\mathrm{m}^{\wedge} 2 / \mathrm{s}$ & $\begin{array}{l}\text { Diffusion } \\
\text { coefficient, } \quad \mathrm{zz} \\
\text { component }\end{array}$ & Domain 1 \\
\hline $\begin{array}{l}\text { chds.Dav_Fcpl } \\
\text { usaq }\end{array}$ & chds.Dxx_Fcplusaq & $\mathrm{m}^{\wedge} 2 / \mathrm{s}$ & $\begin{array}{l}\text { Average } \\
\text { diffusion } \\
\text { coefficient }\end{array}$ & Domain 1 \\
\hline $\begin{array}{l}\text { chds.tfluxx_Fc } \\
\text { plusaq }\end{array}$ & $\begin{array}{l}\text { - } \\
\text { chds.Dxx_Fcplusaq*Fcplus } \\
\text { aqx+chds.cfluxx_Fcplusaq }\end{array}$ & $\mathrm{mol} /\left(\mathrm{m}^{\wedge} 2 * \mathrm{~s}\right)$ & $\begin{array}{l}\text { Total flux, } \mathrm{x} \\
\text { component }\end{array}$ & Domain 1 \\
\hline $\begin{array}{l}\text { chds.tfluxy_Fc } \\
\text { plusaq }\end{array}$ & $\begin{array}{l}\text { - } \\
\text { chds.Dyx_Fcplusaq*Fcplus } \\
\text { aqx+chds.cfluxy_Fcplusaq }\end{array}$ & $\mathrm{mol} /\left(\mathrm{m}^{\wedge} 2 * \mathrm{~s}\right)$ & $\begin{array}{l}\text { Total flux, y } \\
\text { component }\end{array}$ & Domain 1 \\
\hline $\begin{array}{l}\text { chds.tfluxz_Fc } \\
\text { plusaq }\end{array}$ & $\begin{array}{l}\text { - } \\
\text { chds.Dzx_Fcplusaq*Fcplus } \\
\text { aqx+chds.cfluxz_Fcplusaq }\end{array}$ & $\mathrm{mol} /\left(\mathrm{m}^{\wedge} 2 * \mathrm{~s}\right)$ & $\begin{array}{l}\text { Total flux, } \mathrm{z} \\
\text { component }\end{array}$ & Domain 1 \\
\hline $\begin{array}{l}\text { chds.dfluxx_Fc } \\
\text { plusaq }\end{array}$ & $\begin{array}{l}\text { - } \\
\text { chds.Dxx_Fcplusaq*Fcplus } \\
\text { aqx }\end{array}$ & $\mathrm{mol} /\left(\mathrm{m}^{\wedge} 2 * \mathrm{~s}\right)$ & $\begin{array}{l}\text { Diffusive flux, } \\
\text { x component }\end{array}$ & Domain 1 \\
\hline $\begin{array}{l}\text { chds.dfluxy_Fc } \\
\text { plusaq }\end{array}$ & $\begin{array}{l}\text { - } \\
\text { chds.Dyx_Fcplusaq*Fcplus } \\
\text { aqx }\end{array}$ & $\mathrm{mol} /\left(\mathrm{m}^{\wedge} 2 * \mathrm{~s}\right)$ & $\begin{array}{l}\text { Diffusive flux, } \\
\text { y component }\end{array}$ & Domain 1 \\
\hline $\begin{array}{l}\text { chds.dfluxz_Fc } \\
\text { plusaq }\end{array}$ & $\begin{array}{l}\text { - } \\
\text { chds.Dzx_Fcplusaq*Fcplus } \\
\text { aqx }\end{array}$ & $\mathrm{mol} /\left(\mathrm{m}^{\wedge} 2 * \mathrm{~s}\right)$ & $\begin{array}{l}\text { Diffusive flux, } \\
\text { z component }\end{array}$ & Domain 1 \\
\hline $\begin{array}{l}\text { chds.gradx_Fc } \\
\text { plusaq }\end{array}$ & Fcplusaqx & $\mathrm{mol} / \mathrm{m}^{\wedge} 4$ & $\begin{array}{l}\text { Concentration } \\
\text { gradient, } \quad \mathrm{X} \\
\text { component }\end{array}$ & Domain 1 \\
\hline
\end{tabular}




\begin{tabular}{|c|c|c|c|c|}
\hline Name & Expression & Unit & Description & Selection \\
\hline $\begin{array}{l}\text { chds.grady_Fc } \\
\text { plusaq }\end{array}$ & 0 & $\mathrm{~mol} / \mathrm{m}^{\wedge} 4$ & $\begin{array}{l}\text { Concentration } \\
\text { gradient, y } \\
\text { component }\end{array}$ & Domain 1 \\
\hline $\begin{array}{l}\text { chds.gradz_Fcp } \\
\text { lusaq }\end{array}$ & 0 & $\mathrm{~mol} / \mathrm{m}^{\wedge} 4$ & \begin{tabular}{l}
\multicolumn{2}{l}{ Concentration } \\
gradient, $\quad \mathrm{Z}$ \\
component
\end{tabular} & Domain 1 \\
\hline $\begin{array}{l}\text { chds.dfluxMag } \\
\text { _Fcplusaq }\end{array}$ & $\begin{array}{l}\text { sqrt(chds.dfluxx_Fcplusaq^ } \\
\text { 2+chds.dfluxy_Fcplusaq^2 } \\
\text { +chds.dfluxz_Fcplusaq^2) }\end{array}$ & $\mathrm{mol} /\left(\mathrm{m}^{\wedge} 2^{*} \mathrm{~s}\right)$ & $\begin{array}{ll}\text { Diffusive flux } \\
\text { magnitude }\end{array}$ & Domain 1 \\
\hline $\begin{array}{l}\text { chds.tfluxMag_ } \\
\text { Fcplusaq }\end{array}$ & $\begin{array}{l}\text { sqrt(chds.tfluxx_Fcplusaq^} \\
\text { 2+chds.tfluxy_Fcplusaq^2 } \\
\text { +chds.tfluxz_Fcplusaq^2) }\end{array}$ & $\mathrm{mol} /\left(\mathrm{m}^{\wedge} 2 * \mathrm{~s}\right)$ & $\begin{array}{ll}\text { Total flux } \\
\text { magnitude }\end{array}$ & Domain 1 \\
\hline $\begin{array}{l}\text { chds.Dxx_MO } \\
\mathrm{H}\end{array}$ & D_M & $\mathrm{m}^{\wedge} 2 / \mathrm{s}$ & $\begin{array}{l}\text { Diffusion } \\
\text { coefficient, } \mathrm{xx} \\
\text { component }\end{array}$ & Domain 1 \\
\hline $\begin{array}{l}\text { chds.Dyx_MO } \\
\mathrm{H}\end{array}$ & 0 & $\mathrm{~m}^{\wedge} 2 / \mathrm{s}$ & $\begin{array}{l}\text { Diffusion } \\
\text { coefficient, yx } \\
\text { component }\end{array}$ & Domain 1 \\
\hline $\begin{array}{l}\text { chds.Dzx_MO } \\
\mathrm{H}\end{array}$ & 0 & $\mathrm{~m}^{\wedge} 2 / \mathrm{s}$ & $\begin{array}{l}\text { Diffusion } \\
\text { coefficient, } \mathrm{zx} \\
\text { component }\end{array}$ & Domain 1 \\
\hline $\begin{array}{l}\text { chds.Dxy_MO } \\
\text { H }\end{array}$ & 0 & $\mathrm{~m}^{\wedge} 2 / \mathrm{s}$ & $\begin{array}{l}\text { Diffusion } \\
\text { coefficient, } x y \\
\text { component }\end{array}$ & Domain 1 \\
\hline $\begin{array}{l}\text { chds.Dyy_MO } \\
\text { H }\end{array}$ & D_M & $\mathrm{m}^{\wedge} 2 / \mathrm{s}$ & $\begin{array}{l}\text { Diffusion } \\
\text { coefficient, yy } \\
\text { component }\end{array}$ & Domain 1 \\
\hline $\begin{array}{l}\text { chds.Dzy_MO } \\
\text { H }\end{array}$ & 0 & $\mathrm{~m}^{\wedge} 2 / \mathrm{s}$ & $\begin{array}{l}\text { Diffusion } \\
\text { coefficient, zy } \\
\text { component }\end{array}$ & Domain 1 \\
\hline
\end{tabular}




\begin{tabular}{|c|c|c|c|c|}
\hline Name & Expression & Unit & Description & Selection \\
\hline $\begin{array}{l}\text { chds.Dxz_MO } \\
\mathrm{H}\end{array}$ & 0 & $\mathrm{~m}^{\wedge} 2 / \mathrm{s}$ & $\begin{array}{l}\text { Diffusion } \\
\text { coefficient, } \quad \mathrm{xz} \\
\text { component }\end{array}$ & Domain 1 \\
\hline $\begin{array}{l}\text { chds.Dyz_MO } \\
\mathrm{H}\end{array}$ & 0 & $\mathrm{~m}^{\wedge} 2 / \mathrm{s}$ & $\begin{array}{l}\text { Diffusion } \\
\text { coefficient, yz } \\
\text { component }\end{array}$ & Domain 1 \\
\hline $\begin{array}{l}\text { chds.Dzz_MO } \\
\mathrm{H}\end{array}$ & D_M & $\mathrm{m}^{\wedge} 2 / \mathrm{s}$ & $\begin{array}{l}\text { Diffusion } \\
\text { coefficient, } \quad \mathrm{zz} \\
\text { component }\end{array}$ & Domain 1 \\
\hline $\begin{array}{l}\text { chds.Dav_MO } \\
\text { H }\end{array}$ & chds.Dxx_MOH & $\mathrm{m}^{\wedge} 2 / \mathrm{s}$ & $\begin{array}{l}\text { Average } \\
\text { diffusion } \\
\text { coefficient }\end{array}$ & Domain 1 \\
\hline $\begin{array}{l}\text { chds.tfluxx_M } \\
\text { OH }\end{array}$ & $\begin{array}{l}\text { - } \\
\text { chds.Dxx_MOH*MOHx+c } \\
\text { hds.cfluxx_MOH }\end{array}$ & $\mathrm{mol} /\left(\mathrm{m}^{\wedge} 2^{*} \mathrm{~s}\right)$ & $\begin{array}{l}\text { Total flux, } \mathrm{x} \\
\text { component }\end{array}$ & Domain 1 \\
\hline $\begin{array}{l}\text { chds.tfluxy_M } \\
\text { OH }\end{array}$ & $\begin{array}{l}\text { - } \\
\text { chds.Dyx_MOH*MOHx+c } \\
\text { hds.cfluxy_MOH }\end{array}$ & $\mathrm{mol} /\left(\mathrm{m}^{\wedge} 2^{*} \mathrm{~s}\right)$ & $\begin{array}{l}\text { Total flux, y } \\
\text { component }\end{array}$ & Domain 1 \\
\hline $\begin{array}{l}\text { chds.tfluxz_M } \\
\text { OH }\end{array}$ & $\begin{array}{l}\text { - } \\
\text { chds.Dzx_MOH*MOHx+c } \\
\text { hds.cfluxz_MOH }\end{array}$ & $\mathrm{mol} /\left(\mathrm{m}^{\wedge} 2^{*} \mathrm{~s}\right)$ & $\begin{array}{l}\text { Total flux, } \mathrm{z} \\
\text { component }\end{array}$ & Domain 1 \\
\hline $\begin{array}{l}\text { chds.dfluxx_M } \\
\text { OH }\end{array}$ & -chds.Dxx_MOH*MOHx & $\mathrm{mol} /\left(\mathrm{m}^{\wedge} 2^{*} \mathrm{~s}\right)$ & $\begin{array}{l}\text { Diffusive flux, } \\
\text { x component }\end{array}$ & Domain 1 \\
\hline $\begin{array}{l}\text { chds.dfluxy_M } \\
\text { OH }\end{array}$ & -chds.Dyx_MOH*MOHx & $\mathrm{mol} /\left(\mathrm{m}^{\wedge} 2^{*} \mathrm{~s}\right)$ & $\begin{array}{l}\text { Diffusive flux, } \\
\text { y component }\end{array}$ & Domain 1 \\
\hline $\begin{array}{l}\text { chds.dfluxz_M } \\
\mathrm{OH}\end{array}$ & -chds.Dzx_MOH*MOHx & $\mathrm{mol} /\left(\mathrm{m}^{\wedge} 2^{*} \mathrm{~s}\right)$ & $\begin{array}{l}\text { Diffusive flux, } \\
\text { z component }\end{array}$ & Domain 1 \\
\hline
\end{tabular}




\begin{tabular}{|c|c|c|c|c|}
\hline Name & Expression & Unit & Description & Selection \\
\hline $\begin{array}{l}\text { chds.gradx_M } \\
\mathrm{OH}\end{array}$ & MOHx & $\mathrm{mol} / \mathrm{m}^{\wedge} 4$ & $\begin{array}{l}\text { Concentration } \\
\text { gradient, } \quad \mathrm{x} \\
\text { component }\end{array}$ & Domain 1 \\
\hline $\begin{array}{l}\text { chds.grady_M } \\
\mathrm{OH}\end{array}$ & 0 & $\mathrm{~mol} / \mathrm{m}^{\wedge} 4$ & $\begin{array}{l}\text { Concentration } \\
\text { gradient, } \quad \mathrm{y} \\
\text { component }\end{array}$ & Domain 1 \\
\hline $\begin{array}{l}\text { chds.gradz_M } \\
\mathrm{OH}\end{array}$ & 0 & $\mathrm{~mol} / \mathrm{m}^{\wedge} 4$ & $\begin{array}{l}\text { Concentration } \\
\text { gradient, } \quad \mathrm{z} \\
\text { component }\end{array}$ & Domain 1 \\
\hline $\begin{array}{l}\text { chds.dfluxMag } \\
\text { _MOH }\end{array}$ & $\begin{array}{l}\text { sqrt(chds.dfluxx_MOH}{ }^{\wedge} 2+ \\
\text { chds.dfluxy_MOH^2+chds. } \\
\text { dfluxz_MOH^2) }\end{array}$ & $\mathrm{mol} /\left(\mathrm{m}^{\wedge} 2^{*} \mathrm{~s}\right)$ & $\begin{array}{l}\text { Diffusive flux } \\
\text { magnitude }\end{array}$ & Domain 1 \\
\hline $\begin{array}{l}\text { chds.tfluxMag_ } \\
\text { MOH }\end{array}$ & $\begin{array}{l}\text { sqrt(chds.tfluxx_MOH^2+c } \\
\text { hds.tfluxy_MOH^2+chds.tf } \\
\text { luxz_MOH^2) }\end{array}$ & $\mathrm{mol} /\left(\mathrm{m}^{\wedge} 2^{*} \mathrm{~s}\right)$ & $\begin{array}{l}\text { Total flux } \\
\text { magnitude }\end{array}$ & Domain 1 \\
\hline chds.u & model.input.u1 & $\mathrm{m} / \mathrm{s}$ & $\begin{array}{l}\text { Velocity field, } \\
\text { x component }\end{array}$ & Domain 1 \\
\hline chds.v & model.input.u2 & $\mathrm{m} / \mathrm{s}$ & $\begin{array}{l}\text { Velocity field, } \\
\text { y component }\end{array}$ & Domain 1 \\
\hline chds.w & model.input.u3 & $\mathrm{m} / \mathrm{s}$ & $\begin{array}{l}\text { Velocity field, } \\
\text { z component }\end{array}$ & Domain 1 \\
\hline $\begin{array}{l}\text { chds.cfluxx_M } \\
\text { aq }\end{array}$ & Maq*model.input.u1 & $\mathrm{mol} /\left(\mathrm{m}^{\wedge} 2 * \mathrm{~s}\right)$ & $\begin{array}{l}\text { Convective } \\
\text { flux, } \quad \mathrm{x} \\
\text { component }\end{array}$ & Domain 1 \\
\hline $\begin{array}{l}\text { chds.cfluxy_M } \\
\text { aq }\end{array}$ & Maq*model.input.u2 & $\mathrm{mol} /\left(\mathrm{m}^{\wedge} 2 * \mathrm{~s}\right)$ & $\begin{array}{l}\text { Convective } \\
\text { flux, } \quad \mathrm{y} \\
\text { component }\end{array}$ & Domain 1 \\
\hline
\end{tabular}




\begin{tabular}{|c|c|c|c|c|}
\hline Name & Expression & Unit & Description & Selection \\
\hline $\begin{array}{l}\text { chds.cfluxz_M } \\
\text { aq }\end{array}$ & Maq*model.input.u3 & $\mathrm{mol} /\left(\mathrm{m}^{\wedge} 2 * \mathrm{~s}\right)$ & $\begin{array}{l}\text { Convective } \\
\text { flux, } \quad \mathrm{z} \\
\text { component }\end{array}$ & Domain 1 \\
\hline $\begin{array}{l}\text { chds.cfluxMag } \\
\text { _Maq }\end{array}$ & $\begin{array}{l}\text { sqrt(chds.cfluxx_Maq^2+c } \\
\text { hds.cfluxy_Maq^2+chds.cf } \\
\text { luxz_Maq^2) }\end{array}$ & $\mathrm{mol} /\left(\mathrm{m}^{\wedge} 2 * \mathrm{~s}\right)$ & $\begin{array}{l}\text { Convective } \\
\text { flux magnitude }\end{array}$ & Domain 1 \\
\hline $\begin{array}{l}\text { chds.cfluxx_O } \\
\text { Haq }\end{array}$ & OHaq*model.input.u1 & $\mathrm{mol} /\left(\mathrm{m}^{\wedge} 2 * \mathrm{~s}\right)$ & $\begin{array}{l}\text { Convective } \\
\text { flux, } \quad \mathrm{x} \\
\text { component }\end{array}$ & Domain 1 \\
\hline $\begin{array}{l}\text { chds.cfluxy_O } \\
\text { Haq }\end{array}$ & OHaq*model.input.u2 & $\mathrm{mol} /\left(\mathrm{m}^{\wedge} 2 * \mathrm{~s}\right)$ & $\begin{array}{l}\text { Convective } \\
\text { flux, } \quad y \\
\text { component }\end{array}$ & Domain 1 \\
\hline $\begin{array}{l}\text { chds.cfluxz_O } \\
\text { Haq }\end{array}$ & OHaq*model.input.u3 & $\mathrm{mol} /\left(\mathrm{m}^{\wedge} 2 * \mathrm{~s}\right)$ & $\begin{array}{l}\text { Convective } \\
\text { flux, } \quad \mathrm{z} \\
\text { component }\end{array}$ & Domain 1 \\
\hline $\begin{array}{l}\text { chds.cfluxMag } \\
\text { _OHaq }\end{array}$ & $\begin{array}{l}\text { sqrt(chds.cfluxx_OHaq^2+ } \\
\text { chds.cfluxy_OHaq^2+chds. } \\
\text { cfluxz_OHaq^2) }\end{array}$ & $\mathrm{mol} /\left(\mathrm{m}^{\wedge} 2 * \mathrm{~s}\right)$ & $\begin{array}{l}\text { Convective } \\
\text { flux magnitude }\end{array}$ & Domain 1 \\
\hline $\begin{array}{l}\text { chds.cfluxx_Fc } \\
\text { plusaq }\end{array}$ & Fcplusaq*model.input.u1 & $\mathrm{mol} /\left(\mathrm{m}^{\wedge} 2 * \mathrm{~s}\right)$ & $\begin{array}{l}\text { Convective } \\
\text { flux, } \quad \mathrm{x} \\
\text { component }\end{array}$ & Domain 1 \\
\hline $\begin{array}{l}\text { chds.cfluxy_Fc } \\
\text { plusaq }\end{array}$ & Fcplusaq*model.input.u2 & $\mathrm{mol} /\left(\mathrm{m}^{\wedge} 2 * \mathrm{~s}\right)$ & $\begin{array}{ll}\text { Convective } & \\
\text { flux, } \quad y \\
\text { component }\end{array}$ & Domain 1 \\
\hline $\begin{array}{l}\text { chds.cfluxz_Fc } \\
\text { plusaq }\end{array}$ & Fcplusaq*model.input.u3 & $\mathrm{mol} /\left(\mathrm{m}^{\wedge} 2 * \mathrm{~s}\right)$ & $\begin{array}{l}\text { Convective } \\
\text { flux, } \quad \mathrm{z} \\
\text { component }\end{array}$ & Domain 1 \\
\hline $\begin{array}{l}\text { chds.cfluxMag } \\
\text { _Fcplusaq }\end{array}$ & $\begin{array}{l}\text { sqrt(chds.cfluxx_Fcplusaq^ } \\
\text { 2+chds.cfluxy_Fcplusaq^2 } \\
\text { +chds.cfluxz_Fcplusaq^2) }\end{array}$ & $\mathrm{mol} /\left(\mathrm{m}^{\wedge} 2 * \mathrm{~s}\right)$ & $\begin{array}{l}\text { Convective } \\
\text { flux magnitude }\end{array}$ & Domain 1 \\
\hline
\end{tabular}




\begin{tabular}{|c|c|c|c|c|}
\hline Name & Expression & Unit & Description & Selection \\
\hline $\begin{array}{l}\text { chds.cfluxx_M } \\
\mathrm{OH}\end{array}$ & MOH*model.input.u1 & $\mathrm{mol} /\left(\mathrm{m}^{\wedge} 2 * \mathrm{~s}\right)$ & $\begin{array}{l}\text { Convective } \\
\text { flux, } \quad \mathrm{x} \\
\text { component }\end{array}$ & Domain 1 \\
\hline $\begin{array}{l}\text { chds.cfluxy_M } \\
\mathrm{OH}\end{array}$ & MOH*model.input.u2 & $\mathrm{mol} /\left(\mathrm{m}^{\wedge} 2 * \mathrm{~s}\right)$ & $\begin{array}{l}\text { Convective } \\
\text { flux, } \quad \mathrm{y} \\
\text { component }\end{array}$ & Domain 1 \\
\hline $\begin{array}{l}\text { chds.cfluxz_M } \\
\mathrm{OH}\end{array}$ & MOH*model.input.u3 & $\mathrm{mol} /\left(\mathrm{m}^{\wedge} 2 * \mathrm{~s}\right)$ & $\begin{array}{l}\text { Convective } \\
\text { flux, } \quad \mathrm{z} \\
\text { component }\end{array}$ & Domain 1 \\
\hline $\begin{array}{l}\text { chds.cfluxMag } \\
\text { _MOH }\end{array}$ & $\begin{array}{l}\text { sqrt(chds.cfluxx_MOH^2+ } \\
\text { chds.cfluxy_MOH^2+chds. } \\
\text { cfluxz_MOH^2) }\end{array}$ & $\mathrm{mol} /\left(\mathrm{m}^{\wedge} 2 * \mathrm{~s}\right)$ & $\begin{array}{l}\text { Convective } \\
\text { flux magnitude }\end{array}$ & Domain 1 \\
\hline chds.helem & $\mathrm{h}$ & $\mathrm{m}$ & Element size & Domain 1 \\
\hline chds.Res_Maq & chds.u*Maqx-chds.R_Maq & $\mathrm{mol} /\left(\mathrm{m}^{\wedge} 3^{*} \mathrm{~s}\right)$ & $\begin{array}{l}\text { Equation } \\
\text { residual }\end{array}$ & Domain 1 \\
\hline $\begin{array}{l}\text { chds.Res_OHa } \\
\text { q }\end{array}$ & $\begin{array}{l}\text { chds.u*OHaqx- } \\
\text { chds.R_OHaq }\end{array}$ & $\mathrm{mol} /\left(\mathrm{m}^{\wedge} 3^{*} \mathrm{~s}\right)$ & $\begin{array}{l}\text { Equation } \\
\text { residual }\end{array}$ & Domain 1 \\
\hline $\begin{array}{l}\text { chds.Res_Fcpl } \\
\text { usaq }\end{array}$ & $\begin{array}{l}\text { chds.u*Fcplusaqx- } \\
\text { chds.R_Fcplusaq }\end{array}$ & $\mathrm{mol} /\left(\mathrm{m}^{\wedge} 3^{*} \mathrm{~s}\right)$ & $\begin{array}{l}\text { Equation } \\
\text { residual }\end{array}$ & Domain 1 \\
\hline $\begin{array}{l}\text { chds.Res_MO } \\
\mathrm{H}\end{array}$ & $\begin{array}{l}\text { chds.u*MOHx- } \\
\text { chds.R_MOH }\end{array}$ & $\mathrm{mol} /\left(\mathrm{m}^{\wedge} 3^{*} \mathrm{~s}\right)$ & $\begin{array}{l}\text { Equation } \\
\text { residual }\end{array}$ & Domain 1 \\
\hline
\end{tabular}

\section{Shape functions}

\begin{tabular}{|l|l|l|l|l|l|}
\hline Name & Shape function & Unit & Description & Shape frame & Selection \\
\hline Maq & Lagrange (Linear) & $\mathrm{mol} / \mathrm{m}^{\wedge} 3$ & Concentration & Material & Domain 1 \\
\hline OHaq & Lagrange (Linear) & $\mathrm{mol} / \mathrm{m}^{\wedge} 3$ & Concentration & Material & Domain 1 \\
\hline Fcplusaq & Lagrange (Linear) & $\mathrm{mol} / \mathrm{m}^{\wedge} 3$ & Concentration & Material & Domain 1 \\
\hline MOH & Lagrange (Linear) & $\mathrm{mol} / \mathrm{m}^{\wedge} 3$ & Concentration & Material & Domain 1 \\
\hline
\end{tabular}




\begin{tabular}{|l|l|l|}
\hline Weak expression & Integration frame & Selection \\
\hline $\begin{array}{l}\text {-d(Maq,t)*test(Maq)- } \\
\text { chds.Dxx_Maq*Maqx*test(Maqx) }\end{array}$ & Material & Domain 1 \\
\hline $\begin{array}{l}\text {-d(OHaq,t)*test(OHaq)- } \\
\text { chds.Dxx_OHaq*OHaqx*test(OHaqx) }\end{array}$ & Material & Domain 1 \\
\hline $\begin{array}{l}\text {-d(Fcplusaq,t)*test(Fcplusaq)- } \\
\text { chds.Dxx_Fcplusaq*Fcplusaqx*test(Fcplusaqx) }\end{array}$ & Material & Domain 1 \\
\hline $\begin{array}{l}\text {-d(MOH,t)*test(MOH)- } \\
\text { chds.Dxx_MOH*MOHx*test(MOHx) }\end{array}$ & Material & Domain 1 \\
\hline -chds.u*Maqx*test(Maq) & Material & Domain 1 \\
\hline chds.cbf_Maq*test(Maq) & Material & Boundaries 1-2 \\
\hline -chds.u*OHaqx*test(OHaq) & Material & Domain 1 \\
\hline chds.cbf_OHaq*test(OHaq) & Material & Boundaries 1-2 \\
\hline -chds.u*Fcplusaqx*test(Fcplusaq) & Material & Domain 1 \\
\hline chds.cbf_Fcplusaq*test(Fcplusaq) & Material & Boundaries 1-2 \\
\hline -chds.u*MOHx*test(MOH) & Material & Domain 1 \\
\hline chds.cbf_MOH*test(MOH) & Material & Boundaries 1-2 \\
\hline chds.streamline & Material & Domain 1 \\
\hline chds.crosswind & Material & Domain 1 \\
\hline
\end{tabular}


$\longrightarrow$

\begin{tabular}{llllll}
\hline-0.01 & -0.005 & 0 & 0.005 & 0.01
\end{tabular}

No Flux 1

Selection

\begin{tabular}{|l|l|}
\hline Geometric entity level & Boundary \\
\hline Selection & No boundaries \\
\hline
\end{tabular}

Equations

$-\mathbf{n} \cdot \mathbf{N}_{i}=0$

Settings

Settings

\begin{tabular}{|l|l|}
\hline Description & Value \\
\hline Apply for all species & Apply for all species \\
\hline
\end{tabular}

Used products

COMSOL Multiphysics 


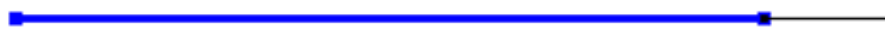

\begin{tabular}{lllllll}
\hline-0.01 & -0.008 & -0.006 & -0.004 & -0.002 & 0
\end{tabular}

Initial Values 1

Selection

\begin{tabular}{|l|l|}
\hline Geometric entity level & Domain \\
\hline Selection & Domain 1 \\
\hline
\end{tabular}

Settings

Settings

\begin{tabular}{|l|l|}
\hline Description & Value \\
\hline Concentration & cMaqi \\
\hline Concentration & cOHaqi \\
\hline Concentration & cDMFcplusaqi \\
\hline Concentration & cMOHi \\
\hline
\end{tabular}

Used products

COMSOL Multiphysics 


\begin{tabular}{llllll}
\hline-1 & -0.5 & 0 & 0.5 & 1
\end{tabular}

Flux 1

Selection

\begin{tabular}{|l|l|}
\hline Geometric entity level & Boundary \\
\hline Selection & Boundary 2 \\
\hline
\end{tabular}

Equations

$-\mathbf{n} \cdot \mathbf{N}_{i}=N_{0 j}$

\section{Settings}

Settings

\begin{tabular}{|l|l|}
\hline Description & Value \\
\hline Species Maq & On \\
\hline Species OHaq & On \\
\hline Species Fcplusaq & On \\
\hline Species MOH & On \\
\hline Inward flux & $\begin{array}{l}\left\{-\mathrm{kb}{ }^{*} \text { Maq }+\mathrm{kf} \text { Mplus, -kb3*OHaq }+\mathrm{kf3} \text { OH, kf2*Fcplus - }\right. \\
\mathrm{kb2} \text { Fcplusaq, kmohf*MOHorg - kmohb*MOH }\}\end{array}$ \\
\hline Flux type & General inward flux \\
\hline
\end{tabular}

Used products

COMSOL Multiphysics 
Variables

\begin{tabular}{|c|c|c|c|c|}
\hline Name & Expression & Unit & Description & \begin{tabular}{|l|} 
Selection \\
\end{tabular} \\
\hline $\begin{array}{l}\text { chds.cbf_- } \\
\text { Maq }\end{array}$ & $\begin{array}{l}\text { Maq*(chds.u*chds.nxmesh+chds. } \\
\text { v*chds.nymesh+chds.w*chds.nz } \\
\text { mesh) }\end{array}$ & $\mathrm{mol} /\left(\mathrm{m}^{\wedge} 2 * \mathrm{~s}\right)$ & $\begin{array}{l}\text { Convective } \\
\text { boundary } \\
\text { flux }\end{array}$ & Boundary 2 \\
\hline $\begin{array}{l}\text { chds.cbf_ } \\
\text { OHaq }\end{array}$ & $\begin{array}{l}\text { OHaq*(chds.u*chds.nxmesh+chd } \\
\text { s.v*chds.nymesh+chds.w*chds.nz } \\
\text { mesh) }\end{array}$ & $\mathrm{mol} /\left(\mathrm{m}^{\wedge} 2^{*} \mathrm{~s}\right)$ & $\begin{array}{l}\text { Convective } \\
\text { boundary } \\
\text { flux }\end{array}$ & Boundary 2 \\
\hline $\begin{array}{l}\text { chds.cbf_ } \\
\text { Fcplusaq }\end{array}$ & $\begin{array}{l}\text { Fcplusaq*(chds.u*chds.nxmesh+c } \\
\text { hds.v*chds.nymesh+chds.w*chds. } \\
\text { nzmesh) }\end{array}$ & $\mathrm{mol} /\left(\mathrm{m}^{\wedge}{ }^{*} * \mathrm{~s}\right)$ & $\begin{array}{l}\text { Convective } \\
\text { boundary } \\
\text { flux }\end{array}$ & Boundary 2 \\
\hline $\begin{array}{l}\text { chds.cbf_ } \\
\mathrm{MOH}\end{array}$ & $\begin{array}{l}\mathrm{MOH}^{*} \text { (chds.u*chds.nxmesh+chds } \\
. \mathrm{v}^{*} \text { chds.nymesh+chds.w*chds.nz } \\
\text { mesh) }\end{array}$ & $\mathrm{mol} /\left(\mathrm{m}^{\wedge} 2^{*} \mathrm{~s}\right)$ & $\begin{array}{l}\text { Convective } \\
\text { boundary } \\
\text { flux }\end{array}$ & Boundary 2 \\
\hline
\end{tabular}

\section{Weak expressions}

\begin{tabular}{|l|l|l|}
\hline Weak expression & Integration frame & Selection \\
\hline$(-\mathrm{kb} *$ Maq+kf*Mplus)*test(Maq) & Material & Boundary 2 \\
\hline (-kb3*OHaq+kf3*OH)*test(OHaq) & Material & Boundary 2 \\
\hline (kf2*Fcplus-kb2*Fcplusaq)*test(Fcplusaq) & Material & Boundary 2 \\
\hline (kmohf*MOHorg-kmohb*MOH)*test(MOH) & Material & Boundary 2 \\
\hline
\end{tabular}




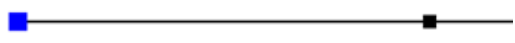

\begin{tabular}{lllll}
\hline-0.02 & -0.015 & -0.01 & -0.005 & 0
\end{tabular}

Concentration 1

Selection

\begin{tabular}{|l|l|}
\hline Geometric entity level & Boundary \\
\hline Selection & Boundary 1 \\
\hline
\end{tabular}

Equations

$c_{i}=c_{0 i}$

Settings

Settings

\begin{tabular}{|l|l|}
\hline Description & Value \\
\hline Concentration & \{Maqi, cOHaqi, cDMFcplusaqi, cMOHi $\}$ \\
\hline Species Maq & On \\
\hline Species OHaq & On \\
\hline Species Fcplusaq & On \\
\hline Species MOH & On \\
\hline Apply reaction terms on & All physics (symmetric) \\
\hline Use weak constraints & Off \\
\hline
\end{tabular}

Used products

COMSOL Multiphysics 
Variables

\begin{tabular}{|l|l|l|l|l|}
\hline Name & Expression & Unit & Description & Selection \\
\hline chds.c0_Maq & cMaqi & $\mathrm{mol} / \mathrm{m}^{\wedge} 3$ & Concentration & Boundary 1 \\
\hline chds.c0_OHaq & cOHaqi & $\mathrm{mol} / \mathrm{m}^{\wedge} 3$ & Concentration & Boundary 1 \\
\hline chds.c0_Fcplusaq & cDMFcplusaqi & $\mathrm{mol} / \mathrm{m}^{\wedge}$ 3 & Concentration & Boundary 1 \\
\hline chds.c0_MOH & cMOHi & $\mathrm{mol} / \mathrm{m}^{\wedge}{ }^{\wedge}$ & Concentration & Boundary 1 \\
\hline
\end{tabular}

\section{Shape functions}

\begin{tabular}{|c|c|c|c|}
\hline Constraint & Constraint force & Shape function & Selection \\
\hline -Maq+chds.c0_Maq & test(-Maq+chds.c0_Maq) & Lagrange (Linear) & Boundary 1 \\
\hline -OHaq+chds.c0_OHaq & $\begin{array}{l}\text { test(- } \\
\text { OHaq+chds.c0_OHaq) }\end{array}$ & Lagrange (Linear) & Boundary 1 \\
\hline $\begin{array}{l}\text { - } \\
\text { Fcplusaq+chds.c0_Fcplus } \\
\text { aq }\end{array}$ & $\begin{array}{l}\text { test(- } \\
\text { Fcplusaq+chds.c0_Fcplus } \\
\text { aq) }\end{array}$ & Lagrange (Linear) & Boundary 1 \\
\hline -MOH+chds.c0_MOH & $\begin{array}{l}\text { test(- } \\
\text { MOH+chds.c0_MOH) }\end{array}$ & Lagrange (Linear) & Boundary 1 \\
\hline
\end{tabular}

\subsubsection{Reactions 1}

$\begin{array}{llllll}-0.01 & -0.008 & -0.006 & -0.004 & -0.002 & 0\end{array}$

Reactions 1

Selection 


\begin{tabular}{|l|l|}
\hline Geometric entity level & Domain \\
\hline Selection & Domain 1 \\
\hline
\end{tabular}

Equations

$$
\frac{\partial c_{i}}{\partial t}+\nabla \cdot\left(-D_{i} \nabla c_{i}\right)+\mathbf{u} \cdot \nabla c_{i}=R_{i}
$$

\section{Settings}

Settings

\begin{tabular}{|l|l|}
\hline Description & Value \\
\hline Total rate expression & $\begin{array}{l}\left\{\mathrm{kmf}^{*} \mathrm{MOH}-\mathrm{kmb}^{*} \mathrm{Maq} * \mathrm{OHaq}, \mathrm{kmf}^{*} \mathrm{MOH}-\mathrm{kmb}^{*} \mathrm{Maq} * \mathrm{OHaq}, 0,\right. \\
\left.-\mathrm{kmf} * \mathrm{MOH}+\mathrm{kmb}^{*} \mathrm{Maq}{ }^{*} \mathrm{OHaq}\right\}\end{array}$ \\
\hline
\end{tabular}

\section{Used products}

COMSOL Multiphysics

\section{Variables}

\begin{tabular}{|c|c|c|c|c|}
\hline Name & Expression & Unit & Description & Selection \\
\hline chds.R_Maq & $\begin{array}{l}\text { kmf*MOH- } \\
\text { kmb*Maq*OHaq }\end{array}$ & $\mathrm{mol} /\left(\mathrm{m}^{\wedge} 3^{*} \mathrm{~s}\right)$ & Total rate expression & Domain 1 \\
\hline chds.R_OHaq & $\begin{array}{l}\text { kmf*MOH- } \\
\text { kmb*Maq*OHaq }\end{array}$ & $\mathrm{mol} /\left(\mathrm{m}^{\wedge} 3^{*} \mathrm{~s}\right)$ & Total rate expression & Domain 1 \\
\hline chds.R_Fcplusaq & 0 & $\mathrm{~mol} /\left(\mathrm{m}^{\wedge} 3^{*} \mathrm{~s}\right)$ & Total rate expression & Domain 1 \\
\hline chds.R_MOH & $\begin{array}{l}- \\
\text { kmf*MOH+kmb*Ma } \\
\text { q*OHaq }\end{array}$ & $\mathrm{mol} /\left(\mathrm{m}^{\wedge} 3^{*} \mathrm{~s}\right)$ & Total rate expression & Domain 1 \\
\hline
\end{tabular}

Weak expressions

\begin{tabular}{|c|c|c|}
\hline Weak expression & Integration frame & Selection \\
\hline (kmf*MOH-kmb*Maq*OHaq)*test(Maq) & Material & Domain 1 \\
\hline (kmf*MOH-kmb*Maq*OHaq)*test(OHaq) & Material & Domain 1 \\
\hline$(-\mathrm{kmf} * \mathrm{MOH}+\mathrm{kmb} * \mathrm{Maq} * \mathrm{OHaq}) * \operatorname{test}(\mathrm{MOH})$ & Material & Domain 1 \\
\hline
\end{tabular}




\subsection{Transport of Diluted Species 2 (chds2)}

\begin{tabular}{llllll}
\hline 0 & 0.002 & 0.004 & 0.006 & 0.008 & 0.01
\end{tabular}

Transport of Diluted Species 2

Selection

\begin{tabular}{|l|l|}
\hline Geometric entity level & Domain \\
\hline Selection & Domain 2 \\
\hline
\end{tabular}

Equations

$$
\begin{aligned}
& \frac{\partial c_{i}}{\partial t}+\nabla \cdot\left(-D_{i} \nabla c_{i}\right)+\mathbf{u} \cdot \nabla c_{i}=R_{i} \\
& \mathbf{N}_{i}=-D_{i} \nabla c_{i}+\mathbf{u} c_{i}
\end{aligned}
$$

Settings

\begin{tabular}{|l|l|}
\hline Description & Value \\
\hline Concentration & Linear \\
\hline Compute boundary fluxes & On \\
\hline Apply smoothing to boundary fluxes & On \\
\hline Value type when using splitting of complex variables & Real \\
\hline Migration in electric field & 0 \\
\hline Convection & 1 \\
\hline Convective term & Non - conservative form \\
\hline Equation residual & Approximate residual \\
\hline Enable space-dependent physics interfaces & 0 \\
\hline
\end{tabular}




\begin{tabular}{|l|l|}
\hline Description & Value \\
\hline Synchronize with COMSOL Multiphysics & \\
\hline
\end{tabular}

\section{Used products}

\begin{tabular}{|l|}
\hline COMSOL Multiphysics \\
\hline Chemical Reaction Engineering Module \\
\hline
\end{tabular}

\begin{tabular}{|c|c|c|c|c|}
\hline Name & Expression & Unit & Description & Selection \\
\hline chds2.nx & unx & & $\begin{array}{l}\text { Normal } \\
\text { vector, } \mathrm{x} \\
\text { component }\end{array}$ & Boundary 2 \\
\hline chds2.ny & 0 & & $\begin{array}{l}\text { Normal } \\
\text { vector, y } \\
\text { component }\end{array}$ & Boundary 2 \\
\hline chds2.nz & 0 & & $\begin{array}{l}\text { Normal } \\
\text { vector, } \quad \mathrm{z} \\
\text { component }\end{array}$ & Boundary 2 \\
\hline chds2.nx & $\operatorname{dn} x$ & & $\begin{array}{l}\text { Normal } \\
\text { vector, } \quad \mathrm{x} \\
\text { component }\end{array}$ & Boundary 3 \\
\hline chds2.ny & 0 & & $\begin{array}{l}\text { Normal } \\
\text { vector, } \quad \mathrm{y} \\
\text { component }\end{array}$ & Boundary 3 \\
\hline chds2.nz & 0 & & $\begin{array}{l}\text { Normal } \\
\text { vector, } \quad \mathrm{z} \\
\text { component }\end{array}$ & Boundary 3 \\
\hline chds2.nxmesh & root.unxmesh & & $\begin{array}{l}\text { Normal } \\
\text { vector } \\
\text { (mesh), x } \\
\text { component }\end{array}$ & Boundary 2 \\
\hline
\end{tabular}




\begin{tabular}{|c|c|c|c|c|}
\hline Name & Expression & Unit & Description & Selection \\
\hline chds2.nymesh & 0 & & $\begin{array}{l}\text { Normal } \\
\text { vector } \\
\text { (mesh), y } \\
\text { component }\end{array}$ & Boundary 2 \\
\hline chds2.nzmesh & 0 & & $\begin{array}{l}\text { Normal } \\
\text { vector } \\
\text { (mesh), z } \\
\text { component }\end{array}$ & Boundary 2 \\
\hline chds2.nxmesh & \begin{tabular}{|l} 
root.dnxmesh \\
\end{tabular} & & $\begin{array}{l}\text { Normal } \\
\text { vector } \\
\text { (mesh), x } \\
\text { component }\end{array}$ & Boundary 3 \\
\hline chds2.nymesh & 0 & & $\begin{array}{l}\text { Normal } \\
\text { vector } \\
\text { (mesh), y } \\
\text { component }\end{array}$ & Boundary 3 \\
\hline chds2.nzmesh & 0 & & $\begin{array}{l}\text { Normal } \\
\text { vector } \\
\text { (mesh), z } \\
\text { component }\end{array}$ & Boundary 3 \\
\hline chds2.R_Mplus & 0 & $\mathrm{~mol} /\left(\mathrm{m}^{\wedge} 3^{*} \mathrm{~s}\right)$ & $\begin{array}{l}\text { Total rate } \\
\text { expression }\end{array}$ & Domain 2 \\
\hline chds2.R_OH & 0 & $\mathrm{~mol} /\left(\mathrm{m}^{\wedge} 3^{*} \mathrm{~s}\right)$ & $\begin{array}{l}\text { Total rate } \\
\text { expression }\end{array}$ & Domain 2 \\
\hline chds2.R_Fc & 0 & $\mathrm{~mol} /\left(\mathrm{m}^{\wedge} 3^{*} \mathrm{~s}\right)$ & $\begin{array}{l}\text { Total rate } \\
\text { expression }\end{array}$ & Domain 2 \\
\hline chds2.R_FcH & 0 & $\mathrm{~mol} /\left(\mathrm{m}^{\wedge} 3^{*} \mathrm{~s}\right)$ & $\begin{array}{l}\text { Total rate } \\
\text { expression }\end{array}$ & Domain 2 \\
\hline chds2.R_Fcplus & 0 & $\mathrm{~mol} /\left(\mathrm{m}^{\wedge} 3^{*} \mathrm{~s}\right)$ & $\begin{array}{l}\text { Total rate } \\
\text { expression }\end{array}$ & Domain 2 \\
\hline
\end{tabular}




\begin{tabular}{|c|c|c|c|c|}
\hline Name & Expression & Unit & Description & Selection \\
\hline chds2.R_MOHorg & 0 & $\mathrm{~mol} /\left(\mathrm{m}^{\wedge} 3^{*} \mathrm{~s}\right)$ & $\begin{array}{l}\text { Total rate } \\
\text { expression }\end{array}$ & Domain 2 \\
\hline chds2.R_O2 & 0 & $\mathrm{~mol} /\left(\mathrm{m}^{\wedge} 3^{*} \mathrm{~s}\right)$ & $\begin{array}{l}\text { Total rate } \\
\text { expression }\end{array}$ & Domain 2 \\
\hline chds2.R_HO2 & 0 & $\mathrm{~mol} /\left(\mathrm{m}^{\wedge} 3^{*} \mathrm{~s}\right)$ & $\begin{array}{l}\text { Total rate } \\
\text { expression }\end{array}$ & Domain 2 \\
\hline chds2.R_HO2minus & 0 & $\mathrm{~mol} /\left(\mathrm{m}^{\wedge} 3^{*} \mathrm{~s}\right)$ & $\begin{array}{l}\text { Total rate } \\
\text { expression }\end{array}$ & Domain 2 \\
\hline chds2.R_H2O2 & 0 & $\mathrm{~mol} /\left(\mathrm{m}^{\wedge} 3^{*} \mathrm{~s}\right)$ & $\begin{array}{l}\text { Total rate } \\
\text { expression }\end{array}$ & Domain 2 \\
\hline domflux.Mplusx & chds2.dfluxx_Mplus & $\mathrm{mol} /\left(\mathrm{m}^{\wedge} 2^{*} \mathrm{~s}\right)$ & Domain flux & Domain 2 \\
\hline domflux.OHx & chds2.dfluxx_OH & $\mathrm{mol} /\left(\mathrm{m}^{\wedge} 2^{*} \mathrm{~s}\right)$ & Domain flux & Domain 2 \\
\hline domflux.Fcx & chds2.dfluxx_Fc & $\mathrm{mol} /\left(\mathrm{m}^{\wedge} 2^{*} \mathrm{~s}\right)$ & Domain flux & Domain 2 \\
\hline domflux.FcHx & chds2.dfluxx_FcH & $\mathrm{mol} /\left(\mathrm{m}^{\wedge} 2^{*} \mathrm{~s}\right)$ & Domain flux & Domain 2 \\
\hline domflux.Fcplusx & chds2.dfluxx_Fcplus & $\mathrm{mol} /\left(\mathrm{m}^{\wedge} 2 * \mathrm{~s}\right)$ & Domain flux & Domain 2 \\
\hline domflux.MOHorgx & $\begin{array}{l}\text { chds2.dfluxx_MOHor } \\
\text { g }\end{array}$ & $\mathrm{mol} /\left(\mathrm{m}^{\wedge} 2^{*} \mathrm{~s}\right)$ & Domain flux & Domain 2 \\
\hline domflux.O2x & chds2.dfluxx_O2 & $\mathrm{mol} /\left(\mathrm{m}^{\wedge} 2 * \mathrm{~s}\right)$ & Domain flux & Domain 2 \\
\hline domflux.HO2x & chds2.dfluxx_HO2 & $\mathrm{mol} /\left(\mathrm{m}^{\wedge} 2 * \mathrm{~s}\right)$ & Domain flux & Domain 2 \\
\hline domflux.HO2minusx & $\begin{array}{l}\text { chds2.dfluxx_HO2mi } \\
\text { nus }\end{array}$ & $\mathrm{mol} /\left(\mathrm{m}^{\wedge} 2 * \mathrm{~s}\right)$ & Domain flux & Domain 2 \\
\hline domflux.H2O2x & chds2.dfluxx_H2O2 & $\mathrm{mol} /\left(\mathrm{m}^{\wedge} 2^{*} \mathrm{~s}\right)$ & Domain flux & Domain 2 \\
\hline chds2.bndFlux_Mplus & -uflux_spatial(Mplus) & $\mathrm{mol} /\left(\mathrm{m}^{\wedge} 2^{*} \mathrm{~s}\right)$ & $\begin{array}{l}\text { Boundary } \\
\text { flux }\end{array}$ & Boundary 2 \\
\hline chds2.bndFlux_Mplus & -dflux_spatial(Mplus) & $\mathrm{mol} /\left(\mathrm{m}^{\wedge} 2 * \mathrm{~s}\right)$ & $\begin{array}{l}\text { Boundary } \\
\text { flux }\end{array}$ & Boundary 3 \\
\hline chds2.ntflux_Mplus & $\begin{array}{l}\text { chds2.bndFlux_Mplus } \\
\text { +chds2.cfluxx_Mplus } \\
\text { *chds2.nx+chds2.cflu }\end{array}$ & $\mathrm{mol} /\left(\mathrm{m}^{\wedge} 2 * \mathrm{~s}\right)$ & $\begin{array}{l}\text { Normal total } \\
\text { flux }\end{array}$ & $\begin{array}{l}\text { Boundaries } \\
2-3\end{array}$ \\
\hline
\end{tabular}




\begin{tabular}{|c|c|c|c|c|}
\hline Name & Expression & Unit & Description & Selection \\
\hline & $\begin{array}{l}\text { xy_Mplus*chds2.ny+c } \\
\text { hds2.cfluxz_Mplus*ch } \\
\text { ds2.nz }\end{array}$ & & & \\
\hline chds2.ndflux_Mplus & chds2.bndFlux_Mplus & $\mathrm{mol} /\left(\mathrm{m}^{\wedge} 2 * \mathrm{~s}\right)$ & $\begin{array}{l}\text { Normal } \\
\text { diffusive flux }\end{array}$ & $\begin{array}{l}\text { Boundaries } \\
2-3\end{array}$ \\
\hline chds2.ncflux_Mplus & $\begin{array}{l}\text { chds2.cfluxx_Mplus*c } \\
\text { hds2.nx+chds2.cfluxy } \\
\text { _Mplus*chds2.ny+chd } \\
\text { s2.cfluxz_Mplus*chds } \\
\text { 2.nz }\end{array}$ & $\mathrm{mol} /\left(\mathrm{m}^{\wedge} 2 * \mathrm{~s}\right)$ & $\begin{array}{l}\text { Normal } \\
\text { convective } \\
\text { flux }\end{array}$ & $\begin{array}{l}\text { Boundaries } \\
2-3\end{array}$ \\
\hline chds2.bndFlux_OH & -uflux_spatial(OH) & $\mathrm{mol} /\left(\mathrm{m}^{\wedge} 2 * \mathrm{~s}\right)$ & $\begin{array}{l}\text { Boundary } \\
\text { flux }\end{array}$ & Boundary 2 \\
\hline chds2.bndFlux_OH & -dflux_spatial(OH) & $\mathrm{mol} /\left(\mathrm{m}^{\wedge} 2 * \mathrm{~s}\right)$ & $\begin{array}{l}\text { Boundary } \\
\text { flux }\end{array}$ & Boundary 3 \\
\hline chds2.ntflux_OH & $\begin{array}{l}\text { chds2.bndFlux_OH+c } \\
\text { hds2.cfluxx_OH*chds } \\
\text { 2.nx+chds2.cfluxy_O } \\
\mathrm{H}^{*} \text { chds2.ny+chds2.cfl } \\
\text { uxz_OH*chds2.nz }\end{array}$ & $\mathrm{mol} /\left(\mathrm{m}^{\wedge} 2^{*} \mathrm{~s}\right)$ & $\begin{array}{l}\text { Normal total } \\
\text { flux }\end{array}$ & $\begin{array}{l}\text { Boundaries } \\
2-3\end{array}$ \\
\hline chds2.ndflux_OH & chds2.bndFlux_OH & $\mathrm{mol} /\left(\mathrm{m}^{\wedge} 2^{*} \mathrm{~s}\right)$ & $\begin{array}{l}\text { Normal } \\
\text { diffusive flux }\end{array}$ & $\begin{array}{l}\text { Boundaries } \\
2-3\end{array}$ \\
\hline chds2.ncflux_OH & $\begin{array}{l}\text { chds2.cfluxx_OH*chd } \\
\text { s2.nx+chds2.cfluxy_O } \\
\mathrm{H}^{*} \text { chds2.ny+chds2.cfl } \\
\text { uxz_OH*chds2.nz }\end{array}$ & $\mathrm{mol} /\left(\mathrm{m}^{\wedge} 2 * \mathrm{~s}\right)$ & $\begin{array}{l}\text { Normal } \\
\text { convective } \\
\text { flux }\end{array}$ & $\begin{array}{l}\text { Boundaries } \\
2-3\end{array}$ \\
\hline chds2.bndFlux_Fc & -uflux_spatial(Fc) & $\mathrm{mol} /\left(\mathrm{m}^{\wedge} 2 * \mathrm{~s}\right)$ & $\begin{array}{l}\text { Boundary } \\
\text { flux }\end{array}$ & Boundary 2 \\
\hline chds2.bndFlux_Fc & -dflux_spatial(Fc) & $\mathrm{mol} /\left(\mathrm{m}^{\wedge} 2 * \mathrm{~s}\right)$ & $\begin{array}{l}\text { Boundary } \\
\text { flux }\end{array}$ & Boundary 3 \\
\hline
\end{tabular}




\begin{tabular}{|c|c|c|c|c|}
\hline Name & Expression & Unit & Description & Selection \\
\hline chds2.ntflux_Fc & $\begin{array}{l}\text { chds2.bndFlux_Fc+ch } \\
\text { ds2.cfluxx_Fc*chds2. } \\
\text { nx+chds2.cfluxy_Fc*c } \\
\text { hds2.ny+chds2.cfluxz } \\
\text { _Fc*chds2.nz }\end{array}$ & $\mathrm{mol} /\left(\mathrm{m}^{\wedge} 2 * \mathrm{~s}\right)$ & $\begin{array}{l}\text { Normal total } \\
\text { flux }\end{array}$ & $\begin{array}{l}\text { Boundaries } \\
2-3\end{array}$ \\
\hline chds2.ndflux_Fc & chds2.bndFlux_Fc & $\mathrm{mol} /\left(\mathrm{m}^{\wedge} 2 * \mathrm{~s}\right)$ & $\begin{array}{l}\text { Normal } \\
\text { diffusive flux }\end{array}$ & $\begin{array}{l}\text { Boundaries } \\
2-3\end{array}$ \\
\hline chds2.ncflux_Fc & $\begin{array}{l}\text { chds2.cfluxx_Fc*chds } \\
\text { 2.nx+chds2.cfluxy_Fc } \\
\text { *chds2.ny+chds2.cflu } \\
\text { xz_Fc*chds2.nz }\end{array}$ & $\mathrm{mol} /\left(\mathrm{m}^{\wedge} 2 * \mathrm{~s}\right)$ & $\begin{array}{l}\text { Normal } \\
\text { convective } \\
\text { flux }\end{array}$ & $\begin{array}{l}\text { Boundaries } \\
2-3\end{array}$ \\
\hline chds2.bndFlux_FcH & -uflux_spatial(FcH) & $\mathrm{mol} /\left(\mathrm{m}^{\wedge} 2^{*} \mathrm{~s}\right)$ & $\begin{array}{l}\text { Boundary } \\
\text { flux }\end{array}$ & Boundary 2 \\
\hline chds2.bndFlux_FcH & -dflux_spatial(FcH) & $\mathrm{mol} /\left(\mathrm{m}^{\wedge} 2 * \mathrm{~s}\right)$ & $\begin{array}{l}\text { Boundary } \\
\text { flux }\end{array}$ & Boundary 3 \\
\hline chds2.ntflux_FcH & $\begin{array}{l}\text { chds2.bndFlux_FcH+c } \\
\text { hds2.cfluxx_FcH*chd } \\
\text { s2.nx+chds2.cfluxy_F } \\
\text { cH*chds2.ny+chds2.c } \\
\text { fluxz_FcH*chds2.nz }\end{array}$ & $\mathrm{mol} /\left(\mathrm{m}^{\wedge} 2 * \mathrm{~s}\right)$ & $\begin{array}{l}\text { Normal total } \\
\text { flux }\end{array}$ & $\begin{array}{l}\text { Boundaries } \\
2-3\end{array}$ \\
\hline chds2.ndflux_FcH & chds2.bndFlux_FcH & $\mathrm{mol} /\left(\mathrm{m}^{\wedge} 2^{*} \mathrm{~s}\right)$ & $\begin{array}{l}\text { Normal } \\
\text { diffusive flux }\end{array}$ & $\begin{array}{l}\text { Boundaries } \\
2-3\end{array}$ \\
\hline chds2.ncflux_FcH & $\begin{array}{l}\text { chds2.cfluxx_FcH*ch } \\
\text { ds2.nx+chds2.cfluxy_ } \\
\text { FcH*chds2.ny+chds2. } \\
\text { cfluxz_FcH*chds2.nz }\end{array}$ & $\mathrm{mol} /\left(\mathrm{m}^{\wedge} 2 * \mathrm{~s}\right)$ & $\begin{array}{l}\text { Normal } \\
\text { convective } \\
\text { flux }\end{array}$ & $\begin{array}{l}\text { Boundaries } \\
2-3\end{array}$ \\
\hline chds2.bndFlux_Fcplus & -uflux_spatial(Fcplus) & $\mathrm{mol} /\left(\mathrm{m}^{\wedge} 2 * \mathrm{~s}\right)$ & $\begin{array}{l}\text { Boundary } \\
\text { flux }\end{array}$ & Boundary 2 \\
\hline chds2.bndFlux_Fcplus & -dflux_spatial(Fcplus) & $\mathrm{mol} /\left(\mathrm{m}^{\wedge} 2 * \mathrm{~s}\right)$ & $\begin{array}{l}\text { Boundary } \\
\text { flux }\end{array}$ & Boundary 3 \\
\hline
\end{tabular}




\begin{tabular}{|c|c|c|c|c|}
\hline Name & Expression & Unit & Description & Selection \\
\hline chds2.ntflux_Fcplus & $\begin{array}{l}\text { chds2.bndFlux_Fcplus } \\
\text { +chds2.cfluxx_Fcplus } \\
\text { *chds2.nx+chds2.cflu } \\
\text { xy_Fcplus*chds2.ny+ } \\
\text { chds2.cfluxz_Fcplus* } \\
\text { chds2.nz }\end{array}$ & $\mathrm{mol} /\left(\mathrm{m}^{\wedge} 2 * \mathrm{~s}\right)$ & $\begin{array}{l}\text { Normal total } \\
\text { flux }\end{array}$ & $\begin{array}{l}\text { Boundaries } \\
2-3\end{array}$ \\
\hline chds2.ndflux_Fcplus & chds2.bndFlux_Fcplus & $\mathrm{mol} /\left(\mathrm{m}^{\wedge} 2 * \mathrm{~s}\right)$ & $\begin{array}{l}\text { Normal } \\
\text { diffusive flux }\end{array}$ & $\begin{array}{l}\text { Boundaries } \\
2-3\end{array}$ \\
\hline chds2.ncflux_Fcplus & $\begin{array}{l}\text { chds2.cfluxx_Fcplus* } \\
\text { chds2.nx+chds2.cflux } \\
\text { y_Fcplus*chds2.ny+c } \\
\text { hds2.cfluxz_Fcplus*c } \\
\text { hds2.nz }\end{array}$ & $\mathrm{mol} /\left(\mathrm{m}^{\wedge} 2 * \mathrm{~s}\right)$ & $\begin{array}{l}\text { Normal } \\
\text { convective } \\
\text { flux }\end{array}$ & $\begin{array}{l}\text { Boundaries } \\
2-3\end{array}$ \\
\hline $\begin{array}{l}\text { chds2.bndFlux_MOH } \\
\text { org }\end{array}$ & $\begin{array}{l}\text { - } \\
\text { uflux_spatial(MOHor } \\
\text { g) }\end{array}$ & $\mathrm{mol} /\left(\mathrm{m}^{\wedge} 2 * \mathrm{~s}\right)$ & $\begin{array}{l}\text { Boundary } \\
\text { flux }\end{array}$ & Boundary 2 \\
\hline $\begin{array}{l}\text { chds2.bndFlux_MOH } \\
\text { org }\end{array}$ & $\begin{array}{l}\text { - } \\
\text { dflux_spatial(MOHor } \\
\text { g) }\end{array}$ & $\mathrm{mol} /\left(\mathrm{m}^{\wedge} 2 * \mathrm{~s}\right)$ & $\begin{array}{l}\text { Boundary } \\
\text { flux }\end{array}$ & Boundary 3 \\
\hline chds2.ntflux_MOHorg & $\begin{array}{l}\text { chds2.bndFlux_MOH } \\
\text { org+chds2.cfluxx_MO } \\
\text { Horg*chds2.nx+chds2 } \\
\text {.cfluxy_MOHorg*chd } \\
\text { s2.ny+chds2.cfluxz_ } \\
\text { MOHorg*chds2.nz }\end{array}$ & $\mathrm{mol} /\left(\mathrm{m}^{\wedge} 2 * \mathrm{~s}\right)$ & $\begin{array}{l}\text { Normal total } \\
\text { flux }\end{array}$ & $\begin{array}{l}\text { Boundaries } \\
2-3\end{array}$ \\
\hline $\begin{array}{l}\text { chds2.ndflux_MOHor } \\
\text { g }\end{array}$ & $\begin{array}{l}\text { chds2.bndFlux_MOH } \\
\text { org }\end{array}$ & $\mathrm{mol} /\left(\mathrm{m}^{\wedge} 2 * \mathrm{~s}\right)$ & $\begin{array}{l}\text { Normal } \\
\text { diffusive flux }\end{array}$ & $\begin{array}{l}\text { Boundaries } \\
2-3\end{array}$ \\
\hline $\begin{array}{l}\text { chds2.ncflux_MOHor } \\
\text { g }\end{array}$ & $\begin{array}{l}\text { chds2.cfluxx_MOHor } \\
\text { g*chds2.nx+chds2.cfl } \\
\text { uxy_MOHorg*chds2. }\end{array}$ & $\mathrm{mol} /\left(\mathrm{m}^{\wedge} 2 * \mathrm{~s}\right)$ & $\begin{array}{l}\text { Normal } \\
\text { convective } \\
\text { flux }\end{array}$ & $\begin{array}{l}\text { Boundaries } \\
2-3\end{array}$ \\
\hline
\end{tabular}




\begin{tabular}{|c|c|c|c|c|}
\hline Name & Expression & Unit & Description & Selection \\
\hline & $\begin{array}{l}\text { ny+chds2.cfluxz_MO } \\
\text { Horg*chds2.nz }\end{array}$ & & & \\
\hline chds2.bndFlux_O2 & -uflux_spatial(O2) & $\mathrm{mol} /\left(\mathrm{m}^{\wedge} 2^{*} \mathrm{~s}\right)$ & $\begin{array}{l}\text { Boundary } \\
\text { flux }\end{array}$ & Boundary 2 \\
\hline chds2.bndFlux_O2 & -dflux_spatial(O2) & $\mathrm{mol} /\left(\mathrm{m}^{\wedge} 2 * \mathrm{~s}\right)$ & $\begin{array}{l}\text { Boundary } \\
\text { flux }\end{array}$ & Boundary 3 \\
\hline chds2.ntflux_O2 & $\begin{array}{l}\text { chds2.bndFlux_O2+ch } \\
\text { ds2.cfluxx_O2*chds2. } \\
\text { nx+chds2.cfluxy_O2* } \\
\text { chds2.ny+chds2.cflux } \\
\text { z_O2*chds2.nz }\end{array}$ & $\mathrm{mol} /\left(\mathrm{m}^{\wedge} 2 * \mathrm{~s}\right)$ & $\begin{array}{l}\text { Normal total } \\
\text { flux }\end{array}$ & $\begin{array}{l}\text { Boundaries } \\
2-3\end{array}$ \\
\hline chds2.ndflux_O2 & chds2.bndFlux_O2 & $\mathrm{mol} /\left(\mathrm{m}^{\wedge} 2 * \mathrm{~s}\right)$ & $\begin{array}{l}\text { Normal } \\
\text { diffusive flux }\end{array}$ & $\begin{array}{l}\text { Boundaries } \\
2-3\end{array}$ \\
\hline chds2.ncflux_O2 & $\begin{array}{l}\text { chds2.cfluxx_O2*chd } \\
\text { s2.nx+chds2.cfluxy_O } \\
2 * \text { chds2.ny+chds2.cfl } \\
\text { uxz_O2*chds2.nz }\end{array}$ & $\mathrm{mol} /\left(\mathrm{m}^{\wedge} 2 * \mathrm{~s}\right)$ & $\begin{array}{l}\text { Normal } \\
\text { convective } \\
\text { flux }\end{array}$ & $\begin{array}{l}\text { Boundaries } \\
2-3\end{array}$ \\
\hline chds2.bndFlux_HO2 & -uflux_spatial(HO2) & $\mathrm{mol} /\left(\mathrm{m}^{\wedge} 2^{*} \mathrm{~s}\right)$ & $\begin{array}{l}\text { Boundary } \\
\text { flux }\end{array}$ & Boundary 2 \\
\hline chds2.bndFlux_HO2 & -dflux_spatial(HO2) & $\mathrm{mol} /\left(\mathrm{m}^{\wedge} 2 * \mathrm{~s}\right)$ & $\begin{array}{l}\text { Boundary } \\
\text { flux }\end{array}$ & Boundary 3 \\
\hline chds2.ntflux_HO2 & $\begin{array}{l}\text { chds2.bndFlux_HO2+ } \\
\text { chds2.cfluxx_HO2*ch } \\
\text { ds2.nx+chds2.cfluxy__ } \\
\text { HO2*chds2.ny+chds2 } \\
\text {.cfluxz_HO2*chds2.n } \\
\text { z }\end{array}$ & $\mathrm{mol} /\left(\mathrm{m}^{\wedge} 2 * \mathrm{~s}\right)$ & $\begin{array}{l}\text { Normal total } \\
\text { flux }\end{array}$ & $\begin{array}{l}\text { Boundaries } \\
2-3\end{array}$ \\
\hline chds2.ndflux_HO2 & chds2.bndFlux_HO2 & $\mathrm{mol} /\left(\mathrm{m}^{\wedge} 2 * \mathrm{~s}\right)$ & $\begin{array}{l}\text { Normal } \\
\text { diffusive flux }\end{array}$ & $\begin{array}{l}\text { Boundaries } \\
2-3\end{array}$ \\
\hline
\end{tabular}




\begin{tabular}{|c|c|c|c|c|}
\hline Name & Expression & Unit & Description & Selection \\
\hline chds2.ncflux_HO2 & $\begin{array}{l}\text { chds2.cfluxx_HO2*ch } \\
\text { ds2.nx+chds2.cfluxy_ } \\
\text { HO2*chds2.ny+chds2 } \\
\text {.cfluxz_HO2*chds2.n } \\
\text { z }\end{array}$ & $\mathrm{mol} /\left(\mathrm{m}^{\wedge} 2^{*} \mathrm{~s}\right)$ & $\begin{array}{l}\text { Normal } \\
\text { convective } \\
\text { flux }\end{array}$ & $\begin{array}{l}\text { Boundaries } \\
2-3\end{array}$ \\
\hline $\begin{array}{l}\text { chds2.bndFlux_HO2m } \\
\text { inus }\end{array}$ & $\begin{array}{l}\text { - } \\
\text { uflux_spatial(HO2min } \\
\text { us) }\end{array}$ & $\mathrm{mol} /\left(\mathrm{m}^{\wedge} 2 * \mathrm{~s}\right)$ & $\begin{array}{l}\text { Boundary } \\
\text { flux }\end{array}$ & Boundary 2 \\
\hline $\begin{array}{l}\text { chds2.bndFlux_HO2m } \\
\text { inus }\end{array}$ & $\begin{array}{l}- \\
\text { dflux_spatial(HO2min } \\
\text { us) }\end{array}$ & $\mathrm{mol} /\left(\mathrm{m}^{\wedge} 2^{*} \mathrm{~s}\right)$ & $\begin{array}{l}\text { Boundary } \\
\text { flux }\end{array}$ & Boundary 3 \\
\hline $\begin{array}{l}\text { chds2.ntflux_HO2min } \\
\text { us }\end{array}$ & $\begin{array}{l}\text { chds2.bndFlux_HO2m } \\
\text { inus+chds2.cfluxx_H } \\
\text { O2minus*chds2.nx+c } \\
\text { hds2.cfluxy_HO2min } \\
\text { us*chds2.ny+chds2.cf } \\
\text { luxz_HO2minus*chds } \\
\text { 2.nz }\end{array}$ & $\mathrm{mol} /\left(\mathrm{m}^{\wedge} 2^{*} \mathrm{~s}\right)$ & $\begin{array}{l}\text { Normal total } \\
\text { flux }\end{array}$ & $\begin{array}{l}\text { Boundaries } \\
2-3\end{array}$ \\
\hline $\begin{array}{l}\text { chds2.ndflux_HO2mi } \\
\text { nus }\end{array}$ & $\begin{array}{l}\text { chds2.bndFlux_HO2m } \\
\text { inus }\end{array}$ & $\mathrm{mol} /\left(\mathrm{m}^{\wedge} 2^{*} \mathrm{~s}\right)$ & $\begin{array}{l}\text { Normal } \\
\text { diffusive flux }\end{array}$ & $\begin{array}{l}\text { Boundaries } \\
2-3\end{array}$ \\
\hline $\begin{array}{l}\text { chds2.ncflux_HO2min } \\
\text { us }\end{array}$ & $\begin{array}{l}\text { chds2.cfluxx_HO2min } \\
\text { us*chds2.nx+chds2.cf } \\
\text { luxy_HO2minus*chds } \\
\text { 2.ny+chds2.cfluxz_H } \\
\text { O2minus*chds2.nz }\end{array}$ & $\mathrm{mol} /\left(\mathrm{m}^{\wedge} 2^{*} \mathrm{~s}\right)$ & $\begin{array}{l}\text { Normal } \\
\text { convective } \\
\text { flux }\end{array}$ & $\begin{array}{l}\text { Boundaries } \\
2-3\end{array}$ \\
\hline chds2.bndFlux_H2O2 & -uflux_spatial(H2O2) & $\mathrm{mol} /\left(\mathrm{m}^{\wedge} 2^{*} \mathrm{~s}\right)$ & $\begin{array}{l}\text { Boundary } \\
\text { flux }\end{array}$ & Boundary 2 \\
\hline chds2.bndFlux_H2O2 & -dflux_spatial(H2O2) & $\mathrm{mol} /\left(\mathrm{m}^{\wedge} 2^{*} \mathrm{~s}\right)$ & $\begin{array}{l}\text { Boundary } \\
\text { flux }\end{array}$ & Boundary 3 \\
\hline
\end{tabular}




\begin{tabular}{|c|c|c|c|c|}
\hline Name & Expression & Unit & Description & Selection \\
\hline chds2.ntflux_H2O2 & $\begin{array}{l}\text { chds2.bndFlux_H2O2 } \\
\text { +chds2.cfluxx_H2O2* } \\
\text { chds2.nx+chds2.cflux } \\
\text { y_H2O2*chds2.ny+ch } \\
\text { ds2.cfluxz_H2O2*chd } \\
\text { s2.nz }\end{array}$ & $\mathrm{mol} /\left(\mathrm{m}^{\wedge} 2^{*} \mathrm{~s}\right)$ & $\begin{array}{l}\text { Normal total } \\
\text { flux }\end{array}$ & $\begin{array}{l}\text { Boundaries } \\
2-3\end{array}$ \\
\hline chds2.ndflux_H2O2 & chds2.bndFlux_H2O2 & $\mathrm{mol} /\left(\mathrm{m}^{\wedge} 2^{*} \mathrm{~s}\right)$ & $\begin{array}{l}\text { Normal } \\
\text { diffusive flux }\end{array}$ & $\begin{array}{l}\text { Boundaries } \\
2-3\end{array}$ \\
\hline chds2.ncflux_H2O2 & $\begin{array}{l}\text { chds2.cfluxx_H2O2*c } \\
\text { hds2.nx+chds2.cfluxy } \\
\text { _H2O2*chds2.ny+chd } \\
\text { s2.cfluxz_H2O2*chds } \\
\text { 2.nz }\end{array}$ & $\mathrm{mol} /\left(\mathrm{m}^{\wedge} 2^{*} \mathrm{~s}\right)$ & $\begin{array}{l}\text { Normal } \\
\text { convective } \\
\text { flux }\end{array}$ & $\begin{array}{l}\text { Boundaries } \\
2-3\end{array}$ \\
\hline
\end{tabular}

\subsubsection{Convection and Diffusion 1}

0

0.002

0.004

0.006

0.008

0.01

Convection and Diffusion 1

Selection

\begin{tabular}{|l|l|}
\hline Geometric entity level & Domain \\
\hline Selection & Domain 2 \\
\hline
\end{tabular}




$$
\begin{aligned}
& \text { Equations } \\
& \qquad \begin{array}{l}
\frac{\partial c_{i}}{\partial t}+\nabla \cdot\left(-D_{i} \nabla c_{i}\right)+\mathbf{u} \cdot \nabla c_{i}=R_{i} \\
\mathbf{N}_{i}=-D_{i} \nabla c_{i}+\mathbf{u} c_{i}
\end{array}
\end{aligned}
$$

\begin{tabular}{|c|c|}
\hline Description & Value \\
\hline Velocity field & User defined \\
\hline Velocity field & $\{0,0,0\}$ \\
\hline Electric potential & User defined \\
\hline Electric potential & 0 \\
\hline Diffusion coefficient & User defined \\
\hline Diffusion coefficient & $\left\{\left\{\mathrm{D} \_\mathrm{M}, 0,0\right\},\left\{0, \mathrm{D} \_\mathrm{M}, 0\right\},\left\{0,0, \mathrm{D} \_\mathrm{M}\right\}\right\}$ \\
\hline Diffusion coefficient & User defined \\
\hline Diffusion coefficient & $\left\{\left\{\mathrm{D} \_\mathrm{Cl}, 0,0\right\},\left\{0, \mathrm{D} \_\mathrm{Cl}, 0\right\},\left\{0,0, \mathrm{D} \_\mathrm{Cl}\right\}\right\}$ \\
\hline Diffusion coefficient & User defined \\
\hline Diffusion coefficient & $\left\{\left\{\mathrm{D} \_\mathrm{DMFc}, 0,0\right\},\left\{0, \mathrm{D} \_\mathrm{DMFc}, 0\right\},\left\{0,0, \mathrm{D} \_\mathrm{DMFc}\right\}\right\}$ \\
\hline Diffusion coefficient & User defined \\
\hline Diffusion coefficient & $\{\{$ D_DMFcplus, 0,0$\},\{0$, D_DMFcplus, 0$\},\{0,0$, D_DMFcplus $\}\}$ \\
\hline Diffusion coefficient & User defined \\
\hline Diffusion coefficient & $\{\{$ D_DMFcplus, 0,0$\},\{0$, D_DMFcplus, 0$\},\{0,0$, D_DMFcplus $\}\}$ \\
\hline Diffusion coefficient & User defined \\
\hline Diffusion coefficient & $\left\{\left\{\mathrm{D} \_\mathrm{M}, 0,0\right\},\left\{0, \mathrm{D} \_\mathrm{M}, 0\right\},\left\{0,0, \mathrm{D} \_\mathrm{M}\right\}\right\}$ \\
\hline Diffusion coefficient & User defined \\
\hline Diffusion coefficient & $\left\{\left\{\mathrm{D} \_\mathrm{O} 2,0,0\right\},\left\{0, \mathrm{D} \_\mathrm{O} 2,0\right\},\left\{0,0, \mathrm{D} \_\mathrm{O} 2\right\}\right\}$ \\
\hline Diffusion coefficient & User defined \\
\hline Diffusion coefficient & $\left\{\left\{\mathrm{D} \_\mathrm{OH}, 0,0\right\},\left\{0, \mathrm{D} \_\mathrm{OH}, 0\right\},\left\{0,0, \mathrm{D} \_\mathrm{OH}\right\}\right\}$ \\
\hline Bulk material & None \\
\hline Diffusion coefficient & User defined \\
\hline Diffusion coefficient & $\left\{\left\{\mathrm{D} \_\mathrm{OH}, 0,0\right\},\left\{0, \mathrm{D} \_\mathrm{OH}, 0\right\},\left\{0,0, \mathrm{D} \_\mathrm{OH}\right\}\right\}$ \\
\hline Diffusion coefficient & User defined \\
\hline
\end{tabular}

\section{Settings}

Settings 


\begin{tabular}{|l|l|}
\hline Description & Value \\
\hline Diffusion coefficient & $\left\{\left\{1 \mathrm{e}-9\left[\mathrm{~m}^{\wedge} \mathrm{2} / \mathrm{s}\right], 0,0\right\},\left\{0,1 \mathrm{e}-9\left[\mathrm{~m}^{\wedge} \mathrm{2} / \mathrm{s}\right], 0\right\},\left\{0,0,1 \mathrm{e}-9\left[\mathrm{~m}^{\wedge} \mathrm{2} / \mathrm{s}\right]\right\}\right\}$ \\
\hline
\end{tabular}

\section{Used products}

\section{COMSOL Multiphysics}

\section{Variables}

\begin{tabular}{|c|c|c|c|c|}
\hline Name & Expression & Unit & Description & Selection \\
\hline $\begin{array}{l}\text { chds2.cbf_Mplu } \\
\text { s }\end{array}$ & 0 & $\mathrm{~mol} /\left(\mathrm{m}^{\wedge} 2 * \mathrm{~s}\right)$ & $\begin{array}{l}\text { Convective } \\
\text { boundary flux }\end{array}$ & $\begin{array}{l}\text { Boundaries } \\
2-3\end{array}$ \\
\hline chds2.cbf_OH & 0 & $\mathrm{~mol} /\left(\mathrm{m}^{\wedge} 2 * \mathrm{~s}\right)$ & $\begin{array}{l}\text { Convective } \\
\text { boundary flux }\end{array}$ & $\begin{array}{l}\text { Boundaries } \\
2-3\end{array}$ \\
\hline chds2.cbf_Fc & 0 & $\mathrm{~mol} /\left(\mathrm{m}^{\wedge} 2^{*} \mathrm{~s}\right)$ & $\begin{array}{l}\text { Convective } \\
\text { boundary flux }\end{array}$ & $\begin{array}{l}\text { Boundaries } \\
2-3\end{array}$ \\
\hline chds2.cbf_FcH & 0 & $\mathrm{~mol} /\left(\mathrm{m}^{\wedge} 2 * \mathrm{~s}\right)$ & $\begin{array}{l}\text { Convective } \\
\text { boundary flux }\end{array}$ & $\begin{array}{l}\text { Boundaries } \\
2-3\end{array}$ \\
\hline $\begin{array}{l}\text { chds2.cbf_Fcplu } \\
\text { s }\end{array}$ & 0 & $\mathrm{~mol} /\left(\mathrm{m}^{\wedge} 2 * \mathrm{~s}\right)$ & $\begin{array}{l}\text { Convective } \\
\text { boundary flux }\end{array}$ & $\begin{array}{l}\text { Boundaries } \\
2-3\end{array}$ \\
\hline $\begin{array}{l}\text { chds2.cbf_MOH } \\
\text { org }\end{array}$ & 0 & $\mathrm{~mol} /\left(\mathrm{m}^{\wedge} 2 * \mathrm{~s}\right)$ & $\begin{array}{l}\text { Convective } \\
\text { boundary flux }\end{array}$ & $\begin{array}{l}\text { Boundaries } \\
2-3\end{array}$ \\
\hline chds2.cbf_O2 & 0 & $\mathrm{~mol} /\left(\mathrm{m}^{\wedge} 2 * \mathrm{~s}\right)$ & $\begin{array}{l}\text { Convective } \\
\text { boundary flux }\end{array}$ & $\begin{array}{l}\text { Boundaries } \\
2-3\end{array}$ \\
\hline chds2.cbf_HO2 & 0 & $\mathrm{~mol} /\left(\mathrm{m}^{\wedge} 2^{*} \mathrm{~s}\right)$ & $\begin{array}{l}\text { Convective } \\
\text { boundary flux }\end{array}$ & $\begin{array}{l}\text { Boundaries } \\
2-3\end{array}$ \\
\hline $\begin{array}{l}\text { chds2.cbf_HO2 } \\
\text { minus }\end{array}$ & 0 & $\mathrm{~mol} /\left(\mathrm{m}^{\wedge} 2^{*} \mathrm{~s}\right)$ & $\begin{array}{l}\text { Convective } \\
\text { boundary flux }\end{array}$ & $\begin{array}{l}\text { Boundaries } \\
2-3\end{array}$ \\
\hline $\begin{array}{l}\text { chds2.cbf_H2O } \\
2\end{array}$ & 0 & $\mathrm{~mol} /\left(\mathrm{m}^{\wedge} 2 * \mathrm{~s}\right)$ & $\begin{array}{l}\text { Convective } \\
\text { boundary flux }\end{array}$ & $\begin{array}{l}\text { Boundaries } \\
2-3\end{array}$ \\
\hline $\begin{array}{l}\text { chds2.Dxx_Mpl } \\
\text { us }\end{array}$ & D_M & $\mathrm{m}^{\wedge} 2 / \mathrm{s}$ & $\begin{array}{l}\text { Diffusion } \\
\text { coefficient, } \mathrm{xx} \\
\text { component }\end{array}$ & Domain 2 \\
\hline
\end{tabular}




\begin{tabular}{|c|c|c|c|c|}
\hline Name & Expression & Unit & Description & Selection \\
\hline $\begin{array}{l}\text { chds2.Dyx_Mpl } \\
\text { us }\end{array}$ & 0 & $\mathrm{~m}^{\wedge} 2 / \mathrm{s}$ & $\begin{array}{l}\text { Diffusion } \\
\text { coefficient, yx } \\
\text { component }\end{array}$ & Domain 2 \\
\hline $\begin{array}{l}\text { chds2.Dzx_Mpl } \\
\text { us }\end{array}$ & 0 & $\mathrm{~m}^{\wedge} 2 / \mathrm{s}$ & $\begin{array}{l}\text { Diffusion } \\
\text { coefficient, zx } \\
\text { component }\end{array}$ & Domain 2 \\
\hline $\begin{array}{l}\text { chds2.Dxy_Mpl } \\
\text { us }\end{array}$ & 0 & $\mathrm{~m}^{\wedge} 2 / \mathrm{s}$ & $\begin{array}{l}\text { Diffusion } \\
\text { coefficient, } \quad x y \\
\text { component }\end{array}$ & Domain 2 \\
\hline $\begin{array}{l}\text { chds2.Dyy_Mpl } \\
\text { us }\end{array}$ & D_M & $\mathrm{m}^{\wedge} 2 / \mathrm{s}$ & $\begin{array}{l}\text { Diffusion } \\
\text { coefficient, yy } \\
\text { component }\end{array}$ & Domain 2 \\
\hline $\begin{array}{l}\text { chds2.Dzy_Mpl } \\
\text { us }\end{array}$ & 0 & $\mathrm{~m}^{\wedge} 2 / \mathrm{s}$ & $\begin{array}{l}\text { Diffusion } \\
\text { coefficient, zy } \\
\text { component }\end{array}$ & Domain 2 \\
\hline $\begin{array}{l}\text { chds2.Dxz_Mpl } \\
\text { us }\end{array}$ & 0 & $\mathrm{~m}^{\wedge} 2 / \mathrm{s}$ & $\begin{array}{l}\text { Diffusion } \\
\text { coefficient, } \quad \mathrm{xz} \\
\text { component }\end{array}$ & Domain 2 \\
\hline $\begin{array}{l}\text { chds2.Dyz_Mpl } \\
\text { us }\end{array}$ & 0 & $\mathrm{~m}^{\wedge} 2 / \mathrm{s}$ & $\begin{array}{l}\text { Diffusion } \\
\text { coefficient, yz } \\
\text { component }\end{array}$ & Domain 2 \\
\hline $\begin{array}{l}\text { chds2.Dzz_Mpl } \\
\text { us }\end{array}$ & D_M & $\mathrm{m}^{\wedge} 2 / \mathrm{s}$ & $\begin{array}{l}\text { Diffusion } \\
\text { coefficient, zz } \\
\text { component }\end{array}$ & Domain 2 \\
\hline $\begin{array}{l}\text { chds2.Dav_Mpl } \\
\text { us }\end{array}$ & chds2.Dxx_Mplus & $\mathrm{m}^{\wedge} 2 / \mathrm{s}$ & $\begin{array}{l}\text { Average } \\
\text { diffusion } \\
\text { coefficient }\end{array}$ & Domain 2 \\
\hline $\begin{array}{l}\text { chds2.tfluxx_M } \\
\text { plus }\end{array}$ & $\begin{array}{l}\text { - } \\
\text { chds2.Dxx_Mplus*Mplus } \\
\text { x+chds2.cfluxx_Mplus }\end{array}$ & $\mathrm{mol} /\left(\mathrm{m}^{\wedge} 2 * \mathrm{~s}\right)$ & $\begin{array}{l}\text { Total flux, } \mathrm{x} \\
\text { component }\end{array}$ & Domain 2 \\
\hline
\end{tabular}




\begin{tabular}{|c|c|c|c|c|}
\hline Name & Expression & Unit & Description & Selection \\
\hline $\begin{array}{l}\text { chds2.tfluxy_M } \\
\text { plus }\end{array}$ & $\begin{array}{l}\text { - } \\
\text { chds2.Dyx_Mplus*Mplus } \\
\text { x+chds2.cfluxy_Mplus }\end{array}$ & $\mathrm{mol} /\left(\mathrm{m}^{\wedge} 2 * \mathrm{~s}\right)$ & $\begin{array}{l}\text { Total flux, y } \\
\text { component }\end{array}$ & Domain 2 \\
\hline $\begin{array}{l}\text { chds2.tfluxz_M } \\
\text { plus }\end{array}$ & $\begin{array}{l}\text { - } \\
\text { chds2.Dzx_Mplus*Mplus } \\
\text { x+chds2.cfluxz_Mplus }\end{array}$ & $\mathrm{mol} /\left(\mathrm{m}^{\wedge} 2 * \mathrm{~s}\right)$ & $\begin{array}{l}\text { Total flux, } \mathrm{z} \\
\text { component }\end{array}$ & Domain 2 \\
\hline $\begin{array}{l}\text { chds2.dfluxx_M } \\
\text { plus }\end{array}$ & $\begin{array}{l}\text { - } \\
\text { chds2.Dxx_Mplus*Mplus } \\
\text { x }\end{array}$ & $\mathrm{mol} /\left(\mathrm{m}^{\wedge} 2^{*} \mathrm{~s}\right)$ & $\begin{array}{l}\text { Diffusive flux, } \\
\text { x component }\end{array}$ & Domain 2 \\
\hline $\begin{array}{l}\text { chds2.dfluxy_M } \\
\text { plus }\end{array}$ & $\begin{array}{l}\text { - } \\
\text { chds2.Dyx_Mplus*Mplus } \\
\mathrm{x}\end{array}$ & $\mathrm{mol} /\left(\mathrm{m}^{\wedge} 2^{*} \mathrm{~s}\right)$ & $\begin{array}{l}\text { Diffusive flux, } \\
\text { y component }\end{array}$ & Domain 2 \\
\hline $\begin{array}{l}\text { chds2.dfluxz_M } \\
\text { plus }\end{array}$ & $\begin{array}{l}\text { - } \\
\text { chds2.Dzx_Mplus*Mplus } \\
\text { x }\end{array}$ & $\mathrm{mol} /\left(\mathrm{m}^{\wedge} 2 * \mathrm{~s}\right)$ & $\begin{array}{l}\text { Diffusive flux, } \\
\text { z component }\end{array}$ & Domain 2 \\
\hline $\begin{array}{l}\text { chds2.gradx_Mp } \\
\text { lus }\end{array}$ & Mplusx & $\mathrm{mol} / \mathrm{m}^{\wedge} 4$ & $\begin{array}{l}\text { Concentration } \\
\text { gradient, } \quad \mathrm{x} \\
\text { component }\end{array}$ & Domain 2 \\
\hline $\begin{array}{l}\text { chds2.grady_Mp } \\
\text { lus }\end{array}$ & 0 & $\mathrm{~mol} / \mathrm{m}^{\wedge} 4$ & $\begin{array}{l}\text { Concentration } \\
\text { gradient, y } \\
\text { component }\end{array}$ & Domain 2 \\
\hline $\begin{array}{l}\text { chds2.gradz_Mp } \\
\text { lus }\end{array}$ & 0 & $\mathrm{~mol} / \mathrm{m}^{\wedge} 4$ & $\begin{array}{l}\text { Concentration } \\
\text { gradient, } \quad \mathrm{Z} \\
\text { component }\end{array}$ & Domain 2 \\
\hline $\begin{array}{l}\text { chds2.dfluxMag } \\
\text { _Mplus }\end{array}$ & $\begin{array}{l}\text { sqrt(chds2.dfluxx_Mplus^ } \\
\text { 2+chds2.dfluxy_Mplus^2 } \\
\text { +chds2.dfluxz_Mplus^2) }\end{array}$ & $\mathrm{mol} /\left(\mathrm{m}^{\wedge} 2 * \mathrm{~s}\right)$ & $\begin{array}{l}\text { Diffusive flux } \\
\text { magnitude }\end{array}$ & Domain 2 \\
\hline $\begin{array}{l}\text { chds2.tfluxMag } \\
\text { _Mplus }\end{array}$ & $\begin{array}{l}\text { sqrt(chds2.tfluxx_Mplus^ } \\
\text { 2+chds2.tfluxy_Mplus`2+ } \\
\text { chds2.tfluxz_Mplus^2) }\end{array}$ & $\mathrm{mol} /\left(\mathrm{m}^{\wedge} 2 * \mathrm{~s}\right)$ & $\begin{array}{ll}\text { Total flux } \\
\text { magnitude }\end{array}$ & Domain 2 \\
\hline
\end{tabular}




\begin{tabular}{|c|c|c|c|c|}
\hline Name & Expression & Unit & Description & Selection \\
\hline chds2.Dxx_OH & D_Cl & $\mathrm{m}^{\wedge} 2 / \mathrm{s}$ & $\begin{array}{l}\text { Diffusion } \\
\text { coefficient, } \mathrm{xx} \\
\text { component }\end{array}$ & Domain 2 \\
\hline chds2.Dyx_OH & 0 & $\mathrm{~m}^{\wedge} 2 / \mathrm{s}$ & $\begin{array}{l}\text { Diffusion } \\
\text { coefficient, yx } \\
\text { component }\end{array}$ & Domain 2 \\
\hline chds2.Dzx_OH & 0 & $\mathrm{~m}^{\wedge} 2 / \mathrm{s}$ & $\begin{array}{l}\text { Diffusion } \\
\text { coefficient, zx } \\
\text { component }\end{array}$ & Domain 2 \\
\hline chds2.Dxy_OH & 0 & $\mathrm{~m}^{\wedge} 2 / \mathrm{s}$ & $\begin{array}{l}\text { Diffusion } \\
\text { coefficient, xy } \\
\text { component }\end{array}$ & Domain 2 \\
\hline chds2.Dyy_OH & D_Cl & $\mathrm{m}^{\wedge} 2 / \mathrm{s}$ & $\begin{array}{l}\text { Diffusion } \\
\text { coefficient, yy } \\
\text { component }\end{array}$ & Domain 2 \\
\hline chds2.Dzy_OH & 0 & $\mathrm{~m}^{\wedge} 2 / \mathrm{s}$ & $\begin{array}{l}\text { Diffusion } \\
\text { coefficient, zy } \\
\text { component }\end{array}$ & Domain 2 \\
\hline chds2.Dxz_OH & 0 & $\mathrm{~m}^{\wedge} 2 / \mathrm{s}$ & $\begin{array}{l}\text { Diffusion } \\
\text { coefficient, } \quad x z \\
\text { component }\end{array}$ & Domain 2 \\
\hline chds2.Dyz_OH & 0 & $\mathrm{~m}^{\wedge} 2 / \mathrm{s}$ & $\begin{array}{l}\text { Diffusion } \\
\text { coefficient, yz } \\
\text { component }\end{array}$ & Domain 2 \\
\hline chds2.Dzz_OH & D_Cl & $\mathrm{m}^{\wedge} 2 / \mathrm{s}$ & $\begin{array}{l}\text { Diffusion } \\
\text { coefficient, zz } \\
\text { component }\end{array}$ & Domain 2 \\
\hline chds2.Dav_OH & chds2.Dxx_OH & $\mathrm{m}^{\wedge} 2 / \mathrm{s}$ & $\begin{array}{l}\text { Average } \\
\text { diffusion } \\
\text { coefficient }\end{array}$ & Domain 2 \\
\hline
\end{tabular}




\begin{tabular}{|c|c|c|c|c|}
\hline Name & Expression & Unit & Description & Selection \\
\hline $\begin{array}{l}\text { chds2.tfluxx_O } \\
\text { H }\end{array}$ & $\begin{array}{l}\text { - } \\
\text { chds2.Dxx_OH*OHx+chd } \\
\text { s2.cfluxx_OH }\end{array}$ & $\mathrm{mol} /\left(\mathrm{m}^{\wedge} 2^{*} \mathrm{~s}\right)$ & $\begin{array}{l}\text { Total flux, } \mathrm{x} \\
\text { component }\end{array}$ & Domain 2 \\
\hline $\begin{array}{l}\text { chds2.tfluxy_O } \\
\text { H }\end{array}$ & $\begin{array}{l}\text { - } \\
\text { chds2.Dyx_OH*OHx+chd } \\
\text { s2.cfluxy_OH }\end{array}$ & $\mathrm{mol} /\left(\mathrm{m}^{\wedge} 2^{*} \mathrm{~s}\right)$ & $\begin{array}{l}\text { Total flux, y } \\
\text { component }\end{array}$ & Domain 2 \\
\hline $\begin{array}{l}\text { chds2.tfluxz_O } \\
\text { H }\end{array}$ & $\begin{array}{l}\text { - } \\
\text { chds2.Dzx_OH*OHx+chd } \\
\text { s2.cfluxz_OH }\end{array}$ & $\mathrm{mol} /\left(\mathrm{m}^{\wedge} 2^{*} \mathrm{~s}\right)$ & $\begin{array}{l}\text { Total flux, } \mathrm{z} \\
\text { component }\end{array}$ & Domain 2 \\
\hline $\begin{array}{l}\text { chds2.dfluxx_O } \\
\text { H }\end{array}$ & -chds2.Dxx_OH*OHx & $\mathrm{mol} /\left(\mathrm{m}^{\wedge} 2^{*} \mathrm{~s}\right)$ & $\begin{array}{l}\text { Diffusive flux, } \\
\text { x component }\end{array}$ & Domain 2 \\
\hline $\begin{array}{l}\text { chds2.dfluxy_O } \\
\text { H }\end{array}$ & -chds2.Dyx_OH*OHx & $\mathrm{mol} /\left(\mathrm{m}^{\wedge} 2^{*} \mathrm{~s}\right)$ & $\begin{array}{l}\text { Diffusive flux, } \\
\text { y component }\end{array}$ & Domain 2 \\
\hline $\begin{array}{l}\text { chds2.dfluxz_O } \\
\text { H }\end{array}$ & -chds2.Dzx_OH*OHx & $\mathrm{mol} /\left(\mathrm{m}^{\wedge} 2 * \mathrm{~s}\right)$ & $\begin{array}{l}\text { Diffusive flux, } \\
\text { z component }\end{array}$ & Domain 2 \\
\hline $\begin{array}{l}\text { chds2.gradx_O } \\
\text { H }\end{array}$ & $\mathrm{OHx}$ & $\mathrm{mol} / \mathrm{m}^{\wedge} 4$ & $\begin{array}{l}\text { Concentration } \\
\text { gradient, } \quad \mathrm{X} \\
\text { component }\end{array}$ & Domain 2 \\
\hline $\begin{array}{l}\text { chds2.grady_O } \\
\text { H }\end{array}$ & 0 & $\mathrm{~mol} / \mathrm{m}^{\wedge} 4$ & $\begin{array}{l}\text { Concentration } \\
\text { gradient, } \quad \mathrm{y} \\
\text { component }\end{array}$ & Domain 2 \\
\hline chds2.gradz_OH & 0 & $\mathrm{~mol} / \mathrm{m}^{\wedge} 4$ & $\begin{array}{l}\text { Concentration } \\
\text { gradient, } \quad \mathrm{z} \\
\text { component }\end{array}$ & Domain 2 \\
\hline $\begin{array}{l}\text { chds2.dfluxMag } \\
\text { _OH }\end{array}$ & $\begin{array}{l}\text { sqrt(chds2.dfluxx_OH^2+ } \\
\text { chds2.dfluxy_OH^2+chds } \\
\text { 2.dfluxz_OH^2) }\end{array}$ & $\mathrm{mol} /\left(\mathrm{m}^{\wedge} 2^{*} \mathrm{~s}\right)$ & $\begin{array}{l}\text { Diffusive flux } \\
\text { magnitude }\end{array}$ & Domain 2 \\
\hline
\end{tabular}




\begin{tabular}{|c|c|c|c|c|}
\hline Name & Expression & Unit & Description & Selection \\
\hline $\begin{array}{l}\text { chds2.tfluxMag } \\
\text { _OH }\end{array}$ & $\begin{array}{l}\text { sqrt(chds2.tfluxx_OH^2+ } \\
\text { chds2.tfluxy_OH^2+chds } \\
\text { 2.tfluxz_OH^2) }\end{array}$ & $\mathrm{mol} /\left(\mathrm{m}^{\wedge} 2 * \mathrm{~s}\right)$ & $\begin{array}{ll}\text { Total flux } \\
\text { magnitude }\end{array}$ & Domain 2 \\
\hline chds2.Dxx_Fc & D_DMFc & $\mathrm{m}^{\wedge} 2 / \mathrm{s}$ & $\begin{array}{l}\text { Diffusion } \\
\text { coefficient, } \mathrm{xx} \\
\text { component }\end{array}$ & Domain 2 \\
\hline chds2.Dyx_Fc & 0 & $\mathrm{~m}^{\wedge} 2 / \mathrm{s}$ & $\begin{array}{l}\text { Diffusion } \\
\text { coefficient, yx } \\
\text { component }\end{array}$ & Domain 2 \\
\hline chds2.Dzx_Fc & 0 & $\mathrm{~m}^{\wedge} 2 / \mathrm{s}$ & $\begin{array}{l}\text { Diffusion } \\
\text { coefficient, } \mathrm{zx} \\
\text { component }\end{array}$ & Domain 2 \\
\hline chds2.Dxy_Fc & 0 & $\mathrm{~m}^{\wedge} 2 / \mathrm{s}$ & $\begin{array}{l}\text { Diffusion } \\
\text { coefficient, } x y \\
\text { component }\end{array}$ & Domain 2 \\
\hline chds2.Dyy_Fc & D_DMFc & $\mathrm{m}^{\wedge} 2 / \mathrm{s}$ & $\begin{array}{l}\text { Diffusion } \\
\text { coefficient, yy } \\
\text { component }\end{array}$ & Domain 2 \\
\hline chds2.Dzy_Fc & 0 & $\mathrm{~m}^{\wedge} 2 / \mathrm{s}$ & $\begin{array}{l}\text { Diffusion } \\
\text { coefficient, zy } \\
\text { component }\end{array}$ & Domain 2 \\
\hline chds2.Dxz_Fc & 0 & $\mathrm{~m}^{\wedge} 2 / \mathrm{s}$ & $\begin{array}{l}\text { Diffusion } \\
\text { coefficient, } \quad \mathrm{xz} \\
\text { component }\end{array}$ & Domain 2 \\
\hline chds2.Dyz_Fc & 0 & $\mathrm{~m}^{\wedge} 2 / \mathrm{s}$ & $\begin{array}{l}\text { Diffusion } \\
\text { coefficient, yz } \\
\text { component }\end{array}$ & Domain 2 \\
\hline chds2.Dzz_Fc & D_DMFc & $\mathrm{m}^{\wedge} 2 / \mathrm{s}$ & $\begin{array}{l}\text { Diffusion } \\
\text { coefficient, } \quad \mathrm{zz} \\
\text { component }\end{array}$ & Domain 2 \\
\hline
\end{tabular}




\begin{tabular}{|c|c|c|c|c|}
\hline Name & Expression & Unit & Description & Selection \\
\hline chds2.Dav_Fc & chds2.Dxx_Fc & $\mathrm{m}^{\wedge} 2 / \mathrm{s}$ & $\begin{array}{l}\text { Average } \\
\text { diffusion } \\
\text { coefficient }\end{array}$ & Domain 2 \\
\hline chds2.tfluxx_Fc & $\begin{array}{l}- \\
\text { chds2.Dxx_Fc*Fcx+chds2 } \\
\text {.cfluxx_Fc }\end{array}$ & $\mathrm{mol} /\left(\mathrm{m}^{\wedge} 2^{*} \mathrm{~s}\right)$ & $\begin{array}{l}\text { Total flux, } \mathrm{x} \\
\text { component }\end{array}$ & Domain 2 \\
\hline chds2.tfluxy_Fc & $\begin{array}{l}- \\
\text { chds2.Dyx_Fc*Fcx+chds2 } \\
\text {.cfluxy_Fc }\end{array}$ & $\mathrm{mol} /\left(\mathrm{m}^{\wedge} 2 * \mathrm{~s}\right)$ & $\begin{array}{l}\text { Total flux, y } \\
\text { component }\end{array}$ & Domain 2 \\
\hline chds2.tfluxz_Fc & $\begin{array}{l}- \\
\text { chds2.Dzx_Fc*Fcx+chds2 } \\
\text {.cfluxz_Fc }\end{array}$ & $\mathrm{mol} /\left(\mathrm{m}^{\wedge} 2 * \mathrm{~s}\right)$ & $\begin{array}{l}\text { Total flux, } \mathrm{z} \\
\text { component }\end{array}$ & Domain 2 \\
\hline chds2.dfluxx_Fc & -chds2.Dxx_Fc*Fcx & $\mathrm{mol} /\left(\mathrm{m}^{\wedge} 2 * \mathrm{~s}\right)$ & $\begin{array}{l}\text { Diffusive flux, } \\
\text { x component }\end{array}$ & Domain 2 \\
\hline chds2.dfluxy_Fc & -chds2.Dyx_Fc*Fcx & $\mathrm{mol} /\left(\mathrm{m}^{\wedge} 2 * \mathrm{~s}\right)$ & $\begin{array}{l}\text { Diffusive flux, } \\
\text { y component }\end{array}$ & Domain 2 \\
\hline chds2.dfluxz_Fc & -chds2.Dzx_Fc*Fcx & $\mathrm{mol} /\left(\mathrm{m}^{\wedge} 2 * \mathrm{~s}\right)$ & $\begin{array}{l}\text { Diffusive flux, } \\
\text { z component }\end{array}$ & Domain 2 \\
\hline chds2.gradx_Fc & Fcx & $\mathrm{mol} / \mathrm{m}^{\wedge} 4$ & $\begin{array}{l}\text { Concentration } \\
\text { gradient, } \quad \mathrm{X} \\
\text { component }\end{array}$ & Domain 2 \\
\hline chds2.grady_Fc & 0 & $\mathrm{~mol} / \mathrm{m}^{\wedge} 4$ & $\begin{array}{l}\text { Concentration } \\
\text { gradient, y } \\
\text { component }\end{array}$ & Domain 2 \\
\hline chds2.gradz_Fc & 0 & $\mathrm{~mol} / \mathrm{m}^{\wedge} 4$ & $\begin{array}{l}\text { Concentration } \\
\text { gradient, } \quad \mathrm{z} \\
\text { component }\end{array}$ & Domain 2 \\
\hline
\end{tabular}




\begin{tabular}{|c|c|c|c|c|}
\hline Name & Expression & Unit & Description & Selection \\
\hline $\begin{array}{l}\text { chds2.dfluxMag } \\
\text { _Fc }\end{array}$ & $\begin{array}{l}\text { sqrt(chds2.dfluxx_Fc^2+c } \\
\text { hds2.dfluxy_Fc^2+chds2. } \\
\text { dfluxz_Fc^2) }\end{array}$ & $\mathrm{mol} /\left(\mathrm{m}^{\wedge} 2 * \mathrm{~s}\right)$ & $\begin{array}{l}\text { Diffusive flux } \\
\text { magnitude }\end{array}$ & Domain 2 \\
\hline $\begin{array}{l}\text { chds2.tfluxMag } \\
\text { _Fc }\end{array}$ & $\begin{array}{l}\text { sqrt(chds2.tfluxx_Fc }{ }^{\wedge} 2+\mathrm{C} \\
\text { hds2.tfluxy_Fc^2+chds2.t } \\
\text { fluxz_Fc^2) }\end{array}$ & $\mathrm{mol} /\left(\mathrm{m}^{\wedge} 2 * \mathrm{~s}\right)$ & $\begin{array}{l}\text { Total flux } \\
\text { magnitude }\end{array}$ & Domain 2 \\
\hline chds2.Dxx_FcH & D_DMFcplus & $\mathrm{m}^{\wedge} 2 / \mathrm{s}$ & $\begin{array}{l}\text { Diffusion } \\
\text { coefficient, } \mathrm{xx} \\
\text { component }\end{array}$ & Domain 2 \\
\hline chds2.Dyx_FcH & 0 & $\mathrm{~m}^{\wedge} 2 / \mathrm{s}$ & $\begin{array}{l}\text { Diffusion } \\
\text { coefficient, } \mathrm{yx} \\
\text { component }\end{array}$ & Domain 2 \\
\hline chds2.Dzx_FcH & 0 & $\mathrm{~m}^{\wedge} 2 / \mathrm{s}$ & $\begin{array}{l}\text { Diffusion } \\
\text { coefficient, } \mathrm{zx} \\
\text { component }\end{array}$ & Domain 2 \\
\hline chds2.Dxy_FcH & 0 & $\mathrm{~m}^{\wedge} 2 / \mathrm{s}$ & $\begin{array}{l}\text { Diffusion } \\
\text { coefficient, xy } \\
\text { component }\end{array}$ & Domain 2 \\
\hline chds2.Dyy_FcH & D_DMFcplus & $\mathrm{m}^{\wedge} 2 / \mathrm{s}$ & $\begin{array}{l}\text { Diffusion } \\
\text { coefficient, yy } \\
\text { component }\end{array}$ & Domain 2 \\
\hline chds2.Dzy_FcH & 0 & $\mathrm{~m}^{\wedge} 2 / \mathrm{s}$ & $\begin{array}{l}\text { Diffusion } \\
\text { coefficient, zy } \\
\text { component }\end{array}$ & Domain 2 \\
\hline chds2.Dxz_FcH & 0 & $\mathrm{~m}^{\wedge} 2 / \mathrm{s}$ & $\begin{array}{l}\text { Diffusion } \\
\text { coefficient, } \mathrm{xz} \\
\text { component }\end{array}$ & Domain 2 \\
\hline chds2.Dyz_FcH & 0 & $\mathrm{~m}^{\wedge} 2 / \mathrm{s}$ & $\begin{array}{l}\text { Diffusion } \\
\text { coefficient, yz } \\
\text { component }\end{array}$ & Domain 2 \\
\hline
\end{tabular}




\begin{tabular}{|c|c|c|c|c|}
\hline Name & Expression & Unit & Description & Selection \\
\hline chds2.Dzz_FcH & D_DMFcplus & $\mathrm{m}^{\wedge} 2 / \mathrm{s}$ & $\begin{array}{l}\text { Diffusion } \\
\text { coefficient, zz } \\
\text { component }\end{array}$ & Domain 2 \\
\hline chds2.Dav_FcH & chds2.Dxx_FcH & $\mathrm{m}^{\wedge} 2 / \mathrm{s}$ & $\begin{array}{l}\text { Average } \\
\text { diffusion } \\
\text { coefficient }\end{array}$ & Domain 2 \\
\hline $\begin{array}{l}\text { chds2.tfluxx_Fc } \\
\text { H }\end{array}$ & $\begin{array}{l}\text { - } \\
\text { chds2.Dxx_FcH*FcHx+c } \\
\text { hds2.cfluxx_FcH }\end{array}$ & $\mathrm{mol} /\left(\mathrm{m}^{\wedge} 2^{*} \mathrm{~s}\right)$ & $\begin{array}{l}\text { Total flux, } \mathrm{x} \\
\text { component }\end{array}$ & Domain 2 \\
\hline $\begin{array}{l}\text { chds2.tfluxy_Fc } \\
\text { H }\end{array}$ & $\begin{array}{l}\text { - } \\
\text { chds2.Dyx_FcH*FcHx+c } \\
\text { hds2.cfluxy_FcH }\end{array}$ & $\mathrm{mol} /\left(\mathrm{m}^{\wedge} 2 * \mathrm{~s}\right)$ & $\begin{array}{l}\text { Total flux, y } \\
\text { component }\end{array}$ & Domain 2 \\
\hline $\begin{array}{l}\text { chds2.tfluxz_Fc } \\
\text { H }\end{array}$ & $\begin{array}{l}\text { - } \\
\text { chds2.Dzx_FcH*FcHx+ch } \\
\text { ds2.cfluxz_FcH }\end{array}$ & $\mathrm{mol} /\left(\mathrm{m}^{\wedge} 2 * \mathrm{~s}\right)$ & $\begin{array}{l}\text { Total flux, } \mathrm{z} \\
\text { component }\end{array}$ & Domain 2 \\
\hline $\begin{array}{l}\text { chds2.dfluxx_Fc } \\
\text { H }\end{array}$ & -chds2.Dxx_FcH*FcHx & $\mathrm{mol} /\left(\mathrm{m}^{\wedge} 2 * \mathrm{~s}\right)$ & $\begin{array}{l}\text { Diffusive flux, } \\
\text { x component }\end{array}$ & Domain 2 \\
\hline $\begin{array}{l}\text { chds2.dfluxy_Fc } \\
\text { H }\end{array}$ & -chds2.Dyx_FcH*FcHx & $\mathrm{mol} /\left(\mathrm{m}^{\wedge} 2^{*} \mathrm{~s}\right)$ & $\begin{array}{l}\text { Diffusive flux, } \\
\text { y component }\end{array}$ & Domain 2 \\
\hline $\begin{array}{l}\text { chds2.dfluxz_Fc } \\
\mathrm{H}\end{array}$ & -chds2.Dzx_FcH*FcHx & $\mathrm{mol} /\left(\mathrm{m}^{\wedge} 2^{*} \mathrm{~s}\right)$ & $\begin{array}{l}\text { Diffusive flux, } \\
\text { z component }\end{array}$ & Domain 2 \\
\hline $\begin{array}{l}\text { chds2.gradx_Fc } \\
\mathrm{H}\end{array}$ & FcHx & $\mathrm{mol} / \mathrm{m}^{\wedge} 4$ & $\begin{array}{l}\text { Concentration } \\
\text { gradient, } \quad \mathrm{X} \\
\text { component }\end{array}$ & Domain 2 \\
\hline $\begin{array}{l}\text { chds2.grady_Fc } \\
\text { H }\end{array}$ & 0 & $\mathrm{~mol} / \mathrm{m}^{\wedge} 4$ & $\begin{array}{l}\text { Concentration } \\
\text { gradient, y } \\
\text { component }\end{array}$ & Domain 2 \\
\hline
\end{tabular}




\begin{tabular}{|c|c|c|c|c|}
\hline Name & Expression & Unit & Description & Selection \\
\hline $\begin{array}{l}\text { chds2.gradz_Fc } \\
\mathrm{H}\end{array}$ & 0 & $\mathrm{~mol} / \mathrm{m}^{\wedge} 4$ & $\begin{array}{l}\text { Concentration } \\
\text { gradient, } \quad \mathrm{Z} \\
\text { component }\end{array}$ & Domain 2 \\
\hline $\begin{array}{l}\text { chds2.dfluxMag } \\
\text { _FcH }\end{array}$ & $\begin{array}{l}\text { sqrt(chds2.dfluxx_FcH^2 } \\
\text { +chds2.dfluxy_FcH^2+ch } \\
\text { ds2.dfluxz_FcH^2) }\end{array}$ & $\mathrm{mol} /\left(\mathrm{m}^{\wedge} 2 * \mathrm{~s}\right)$ & $\begin{array}{ll}\text { Diffusive flux } \\
\text { magnitude }\end{array}$ & Domain 2 \\
\hline $\begin{array}{l}\text { chds2.tfluxMag } \\
\text { _FcH }\end{array}$ & $\begin{array}{l}\text { sqrt(chds2.tfluxx_FcH }{ }^{\wedge} 2+ \\
\text { chds2.tfluxy_FcH^2+chds } \\
\text { 2.tfluxz_FcH^2) }\end{array}$ & $\mathrm{mol} /\left(\mathrm{m}^{\wedge} 2^{*} \mathrm{~s}\right)$ & $\begin{array}{ll}\text { Total flux } \\
\text { magnitude }\end{array}$ & Domain 2 \\
\hline $\begin{array}{l}\text { chds2.Dxx_Fcpl } \\
\text { us }\end{array}$ & D_DMFcplus & $\mathrm{m}^{\wedge} 2 / \mathrm{s}$ & $\begin{array}{l}\text { Diffusion } \\
\text { coefficient, } \mathrm{xx} \\
\text { component }\end{array}$ & Domain 2 \\
\hline $\begin{array}{l}\text { chds2.Dyx_Fcpl } \\
\text { us }\end{array}$ & 0 & $\mathrm{~m}^{\wedge} 2 / \mathrm{s}$ & $\begin{array}{l}\text { Diffusion } \\
\text { coefficient, yx } \\
\text { component }\end{array}$ & Domain 2 \\
\hline $\begin{array}{l}\text { chds2.Dzx_Fcpl } \\
\text { us }\end{array}$ & 0 & $\mathrm{~m}^{\wedge} 2 / \mathrm{s}$ & $\begin{array}{l}\text { Diffusion } \\
\text { coefficient, zx } \\
\text { component }\end{array}$ & Domain 2 \\
\hline $\begin{array}{l}\text { chds2.Dxy_Fcpl } \\
\text { us }\end{array}$ & 0 & $\mathrm{~m}^{\wedge} 2 / \mathrm{s}$ & $\begin{array}{l}\text { Diffusion } \\
\text { coefficient, } \quad x y \\
\text { component }\end{array}$ & Domain 2 \\
\hline $\begin{array}{l}\text { chds2.Dyy_Fcpl } \\
\text { us }\end{array}$ & D_DMFcplus & $\mathrm{m}^{\wedge} 2 / \mathrm{s}$ & $\begin{array}{l}\text { Diffusion } \\
\text { coefficient, yy } \\
\text { component }\end{array}$ & Domain 2 \\
\hline $\begin{array}{l}\text { chds2.Dzy_Fcpl } \\
\text { us }\end{array}$ & 0 & $m^{\wedge} 2 / s$ & $\begin{array}{l}\text { Diffusion } \\
\text { coefficient, zy } \\
\text { component }\end{array}$ & Domain 2 \\
\hline $\begin{array}{l}\text { chds2.Dxz_Fcpl } \\
\text { us }\end{array}$ & 0 & $\mathrm{~m}^{\wedge} 2 / \mathrm{s}$ & $\begin{array}{l}\text { Diffusion } \\
\text { coefficient, } \quad \mathrm{xz} \\
\text { component }\end{array}$ & Domain 2 \\
\hline
\end{tabular}




\begin{tabular}{|c|c|c|c|c|}
\hline Name & Expression & Unit & Description & Selection \\
\hline $\begin{array}{l}\text { chds2.Dyz_Fcpl } \\
\text { us }\end{array}$ & 0 & $\mathrm{~m}^{\wedge} 2 / \mathrm{s}$ & $\begin{array}{l}\text { Diffusion } \\
\text { coefficient, yz } \\
\text { component }\end{array}$ & Domain 2 \\
\hline $\begin{array}{l}\text { chds2.Dzz_Fcpl } \\
\text { us }\end{array}$ & D_DMFcplus & $\mathrm{m}^{\wedge} 2 / \mathrm{s}$ & $\begin{array}{l}\text { Diffusion } \\
\text { coefficient, } \quad \mathrm{zz} \\
\text { component }\end{array}$ & Domain 2 \\
\hline $\begin{array}{l}\text { chds2.Dav_Fcpl } \\
\text { us }\end{array}$ & chds2.Dxx_Fcplus & $\mathrm{m}^{\wedge} 2 / \mathrm{s}$ & $\begin{array}{l}\text { Average } \\
\text { diffusion } \\
\text { coefficient }\end{array}$ & Domain 2 \\
\hline $\begin{array}{l}\text { chds2.tfluxx_Fc } \\
\text { plus }\end{array}$ & $\begin{array}{l}\text { - } \\
\text { chds2.Dxx_Fcplus*Fcplus } \\
\text { x+chds2.cfluxx_Fcplus }\end{array}$ & $\mathrm{mol} /\left(\mathrm{m}^{\wedge} 2 * \mathrm{~s}\right)$ & $\begin{array}{l}\text { Total flux, } \mathrm{x} \\
\text { component }\end{array}$ & Domain 2 \\
\hline $\begin{array}{l}\text { chds2.tfluxy_Fc } \\
\text { plus }\end{array}$ & $\begin{array}{l}\text { - } \\
\text { chds2.Dyx_Fcplus*Fcplus } \\
\text { x+chds2.cfluxy_Fcplus }\end{array}$ & $\mathrm{mol} /\left(\mathrm{m}^{\wedge} 2 * \mathrm{~s}\right)$ & $\begin{array}{l}\text { Total flux, y } \\
\text { component }\end{array}$ & Domain 2 \\
\hline $\begin{array}{l}\text { chds2.tfluxz_Fc } \\
\text { plus }\end{array}$ & $\begin{array}{l}\text { - } \\
\text { chds2.Dzx_Fcplus*Fcplus } \\
\text { x+chds2.cfluxz_Fcplus }\end{array}$ & $\mathrm{mol} /\left(\mathrm{m}^{\wedge} 2 * \mathrm{~s}\right)$ & $\begin{array}{l}\text { Total flux, } \mathrm{z} \\
\text { component }\end{array}$ & Domain 2 \\
\hline $\begin{array}{l}\text { chds2.dfluxx_Fc } \\
\text { plus }\end{array}$ & $\begin{array}{l}\text { - } \\
\text { chds2.Dxx_Fcplus*Fcplus } \\
\text { x }\end{array}$ & $\mathrm{mol} /\left(\mathrm{m}^{\wedge} 2 * \mathrm{~s}\right)$ & $\begin{array}{l}\text { Diffusive flux, } \\
\text { x component }\end{array}$ & Domain 2 \\
\hline $\begin{array}{l}\text { chds2.dfluxy_Fc } \\
\text { plus }\end{array}$ & $\begin{array}{l}\text { - } \\
\text { chds2.Dyx_Fcplus*Fcplus } \\
\mathrm{x}\end{array}$ & $\mathrm{mol} /\left(\mathrm{m}^{\wedge} 2 * \mathrm{~s}\right)$ & $\begin{array}{l}\text { Diffusive flux, } \\
\text { y component }\end{array}$ & Domain 2 \\
\hline $\begin{array}{l}\text { chds2.dfluxz_Fc } \\
\text { plus }\end{array}$ & $\begin{array}{l}\text { - } \\
\text { chds2.Dzx_Fcplus*Fcplus } \\
\text { x }\end{array}$ & $\mathrm{mol} /\left(\mathrm{m}^{\wedge} 2 * \mathrm{~s}\right)$ & $\begin{array}{l}\text { Diffusive flux, } \\
\text { z component }\end{array}$ & Domain 2 \\
\hline $\begin{array}{l}\text { chds2.gradx_Fc } \\
\text { plus }\end{array}$ & Fcplusx & $\mathrm{mol} / \mathrm{m}^{\wedge} 4$ & $\begin{array}{l}\text { Concentration } \\
\text { gradient, } \quad \mathrm{X} \\
\text { component }\end{array}$ & Domain 2 \\
\hline
\end{tabular}




\begin{tabular}{|c|c|c|c|c|}
\hline Name & Expression & Unit & Description & Selection \\
\hline $\begin{array}{l}\text { chds2.grady_Fc } \\
\text { plus }\end{array}$ & 0 & $\mathrm{~mol} / \mathrm{m}^{\wedge} 4$ & $\begin{array}{l}\text { Concentration } \\
\text { gradient, } \quad \mathrm{y} \\
\text { component }\end{array}$ & Domain 2 \\
\hline $\begin{array}{l}\text { chds2.gradz_Fc } \\
\text { plus }\end{array}$ & 0 & $\mathrm{~mol} / \mathrm{m}^{\wedge} 4$ & $\begin{array}{l}\text { Concentration } \\
\text { gradient, } \quad \mathrm{Z} \\
\text { component }\end{array}$ & Domain 2 \\
\hline $\begin{array}{l}\text { chds2.dfluxMag } \\
\text { _Fcplus }\end{array}$ & $\begin{array}{l}\text { sqrt(chds2.dfluxx_Fcplus^ } \\
\text { 2+chds2.dfluxy_Fcplus`2 } \\
\text { +chds2.dfluxz_Fcplus^2) }\end{array}$ & $\mathrm{mol} /\left(\mathrm{m}^{\wedge} 2 * \mathrm{~s}\right)$ & $\begin{array}{l}\text { Diffusive flux } \\
\text { magnitude }\end{array}$ & Domain 2 \\
\hline $\begin{array}{l}\text { chds2.tfluxMag } \\
\text { _Fcplus }\end{array}$ & $\begin{array}{l}\text { sqrt(chds2.tfluxx_Fcplus` } \\
\text { 2+chds2.tfluxy_Fcplus`2 } \\
\text { +chds2.tfluxz_Fcplus`2) }\end{array}$ & $\mathrm{mol} /\left(\mathrm{m}^{\wedge} 2 * \mathrm{~s}\right)$ & $\begin{array}{ll}\text { Total flux } \\
\text { magnitude }\end{array}$ & Domain 2 \\
\hline $\begin{array}{l}\text { chds2.Dxx_MO } \\
\text { Horg }\end{array}$ & D_M & $\mathrm{m}^{\wedge} 2 / \mathrm{s}$ & $\begin{array}{l}\text { Diffusion } \\
\text { coefficient, } \mathrm{xx} \\
\text { component }\end{array}$ & Domain 2 \\
\hline $\begin{array}{l}\text { chds2.Dyx_MO } \\
\text { Horg }\end{array}$ & 0 & $\mathrm{~m}^{\wedge} 2 / \mathrm{s}$ & $\begin{array}{l}\text { Diffusion } \\
\text { coefficient, yx } \\
\text { component }\end{array}$ & Domain 2 \\
\hline $\begin{array}{l}\text { chds2.Dzx_MO } \\
\text { Horg }\end{array}$ & 0 & $\mathrm{~m}^{\wedge} 2 / \mathrm{s}$ & $\begin{array}{l}\text { Diffusion } \\
\text { coefficient, zx } \\
\text { component }\end{array}$ & Domain 2 \\
\hline $\begin{array}{l}\text { chds2.Dxy_MO } \\
\text { Horg }\end{array}$ & 0 & $\mathrm{~m}^{\wedge} 2 / \mathrm{s}$ & $\begin{array}{l}\text { Diffusion } \\
\text { coefficient, } x y \\
\text { component }\end{array}$ & Domain 2 \\
\hline $\begin{array}{l}\text { chds2.Dyy_MO } \\
\text { Horg }\end{array}$ & D_M & $\mathrm{m}^{\wedge} 2 / \mathrm{s}$ & $\begin{array}{l}\text { Diffusion } \\
\text { coefficient, yy } \\
\text { component }\end{array}$ & Domain 2 \\
\hline $\begin{array}{l}\text { chds2.Dzy_MO } \\
\text { Horg }\end{array}$ & 0 & $\mathrm{~m}^{\wedge} 2 / \mathrm{s}$ & $\begin{array}{l}\text { Diffusion } \\
\text { coefficient, zy } \\
\text { component }\end{array}$ & Domain 2 \\
\hline
\end{tabular}




\begin{tabular}{|c|c|c|c|c|}
\hline Name & Expression & Unit & Description & Selection \\
\hline $\begin{array}{l}\text { chds2.Dxz_MO } \\
\text { Horg }\end{array}$ & 0 & $\mathrm{~m}^{\wedge} 2 / \mathrm{s}$ & $\begin{array}{l}\text { Diffusion } \\
\text { coefficient, } \quad \mathrm{xz} \\
\text { component }\end{array}$ & Domain 2 \\
\hline $\begin{array}{l}\text { chds2.Dyz_MO } \\
\text { Horg }\end{array}$ & 0 & $\mathrm{~m}^{\wedge} 2 / \mathrm{s}$ & $\begin{array}{l}\text { Diffusion } \\
\text { coefficient, yz } \\
\text { component }\end{array}$ & Domain 2 \\
\hline $\begin{array}{l}\text { chds2.Dzz_MO } \\
\text { Horg }\end{array}$ & D_M & $\mathrm{m}^{\wedge} 2 / \mathrm{s}$ & $\begin{array}{l}\text { Diffusion } \\
\text { coefficient, } \quad \mathrm{zz} \\
\text { component }\end{array}$ & Domain 2 \\
\hline $\begin{array}{l}\text { chds2.Dav_MO } \\
\text { Horg }\end{array}$ & chds2.Dxx_MOHorg & $\mathrm{m}^{\wedge} 2 / \mathrm{s}$ & $\begin{array}{l}\text { Average } \\
\text { diffusion } \\
\text { coefficient }\end{array}$ & Domain 2 \\
\hline $\begin{array}{l}\text { chds2.tfluxx_M } \\
\text { OHorg }\end{array}$ & $\begin{array}{l}\text { - } \\
\text { chds2.Dxx_MOHorg*MO } \\
\text { Horgx+chds2.cfluxx_MO } \\
\text { Horg }\end{array}$ & $\mathrm{mol} /\left(\mathrm{m}^{\wedge} 2 * \mathrm{~s}\right)$ & $\begin{array}{l}\text { Total flux, } \mathrm{x} \\
\text { component }\end{array}$ & Domain 2 \\
\hline $\begin{array}{l}\text { chds2.tfluxy_M } \\
\text { OHorg }\end{array}$ & $\begin{array}{l}\text { - } \\
\text { chds2.Dyx_MOHorg*MO } \\
\text { Horgx+chds2.cfluxy_MO } \\
\text { Horg }\end{array}$ & $\mathrm{mol} /\left(\mathrm{m}^{\wedge} 2 * \mathrm{~s}\right)$ & $\begin{array}{l}\text { Total flux, y } \\
\text { component }\end{array}$ & Domain 2 \\
\hline $\begin{array}{l}\text { chds2.tfluxz_M } \\
\text { OHorg }\end{array}$ & $\begin{array}{l}\text { - } \\
\text { chds2.Dzx_MOHorg*MO } \\
\text { Horgx+chds2.cfluxz_MO } \\
\text { Horg }\end{array}$ & $\mathrm{mol} /\left(\mathrm{m}^{\wedge} 2^{*} \mathrm{~s}\right)$ & $\begin{array}{l}\text { Total flux, } \mathrm{z} \\
\text { component }\end{array}$ & Domain 2 \\
\hline $\begin{array}{l}\text { chds2.dfluxx_M } \\
\text { OHorg }\end{array}$ & $\begin{array}{l}\text { - } \\
\text { chds2.Dxx_MOHorg*MO } \\
\text { Horgx }\end{array}$ & $\mathrm{mol} /\left(\mathrm{m}^{\wedge} 2 * \mathrm{~s}\right)$ & $\begin{array}{l}\text { Diffusive flux, } \\
\text { x component }\end{array}$ & Domain 2 \\
\hline $\begin{array}{l}\text { chds2.dfluxy_M } \\
\text { OHorg }\end{array}$ & $\begin{array}{l}\text { - } \\
\text { chds2.Dyx_MOHorg*MO } \\
\text { Horgx }\end{array}$ & $\mathrm{mol} /\left(\mathrm{m}^{\wedge} 2 * \mathrm{~s}\right)$ & $\begin{array}{l}\text { Diffusive flux, } \\
\text { y component }\end{array}$ & Domain 2 \\
\hline
\end{tabular}




\begin{tabular}{|c|c|c|c|c|}
\hline Name & Expression & Unit & Description & Selection \\
\hline $\begin{array}{l}\text { chds2.dfluxz_M } \\
\text { OHorg }\end{array}$ & $\begin{array}{l}\text { - } \\
\text { chds2.Dzx_MOHorg*MO } \\
\text { Horgx }\end{array}$ & $\mathrm{mol} /\left(\mathrm{m}^{\wedge} 2 * \mathrm{~s}\right)$ & $\begin{array}{l}\text { Diffusive flux, } \\
\text { z component }\end{array}$ & Domain 2 \\
\hline $\begin{array}{l}\text { chds2.gradx_M } \\
\text { OHorg }\end{array}$ & MOHorgx & $\mathrm{mol} / \mathrm{m}^{\wedge} 4$ & $\begin{array}{l}\text { Concentration } \\
\text { gradient, } \quad \mathrm{X} \\
\text { component }\end{array}$ & Domain 2 \\
\hline $\begin{array}{l}\text { chds2.grady_M } \\
\text { OHorg }\end{array}$ & 0 & $\mathrm{~mol} / \mathrm{m}^{\wedge} 4$ & $\begin{array}{l}\text { Concentration } \\
\text { gradient, } \quad \mathrm{y} \\
\text { component }\end{array}$ & Domain 2 \\
\hline $\begin{array}{l}\text { chds2.gradz_M } \\
\text { OHorg }\end{array}$ & 0 & $\mathrm{~mol} / \mathrm{m}^{\wedge} 4$ & $\begin{array}{l}\text { Concentration } \\
\text { gradient, } \quad \mathrm{Z} \\
\text { component }\end{array}$ & Domain 2 \\
\hline $\begin{array}{l}\text { chds2.dfluxMag } \\
\text { _MOHorg }\end{array}$ & $\begin{array}{l}\text { sqrt(chds2.dfluxx_MOHor } \\
\mathrm{g}^{\wedge} 2+\text { chds2.dfluxy_MOHo } \\
\text { rg^2+chds2.dfluxz_MOH } \\
\text { org^2) }\end{array}$ & $\mathrm{mol} /\left(\mathrm{m}^{\wedge} 2 * \mathrm{~s}\right)$ & $\begin{array}{ll}\text { Diffusive flux } \\
\text { magnitude }\end{array}$ & Domain 2 \\
\hline $\begin{array}{l}\text { chds2.tfluxMag } \\
\text { _MOHorg }\end{array}$ & $\begin{array}{l}\text { sqrt(chds2.tfluxx_MOHor } \\
\mathrm{g}^{\wedge} 2+\text { chds2.tfluxy_MOHor } \\
\mathrm{g}^{\wedge} 2+\text { chds2.tfluxz_MOHor } \\
\left.\mathrm{g}^{\wedge} 2\right)\end{array}$ & $\mathrm{mol} /\left(\mathrm{m}^{\wedge} 2 * \mathrm{~s}\right)$ & $\begin{array}{ll}\text { Total flux } \\
\text { magnitude }\end{array}$ & Domain 2 \\
\hline chds2.Dxx_O2 & D_O2 & $\mathrm{m}^{\wedge} 2 / \mathrm{s}$ & $\begin{array}{l}\text { Diffusion } \\
\text { coefficient, } \mathrm{xx} \\
\text { component }\end{array}$ & Domain 2 \\
\hline chds2.Dyx_O2 & 0 & $\mathrm{~m}^{\wedge} 2 / \mathrm{s}$ & $\begin{array}{l}\text { Diffusion } \\
\text { coefficient, yx } \\
\text { component }\end{array}$ & Domain 2 \\
\hline chds2.Dzx_O2 & 0 & $\mathrm{~m}^{\wedge} 2 / \mathrm{s}$ & $\begin{array}{l}\text { Diffusion } \\
\text { coefficient, zx } \\
\text { component }\end{array}$ & Domain 2 \\
\hline
\end{tabular}




\begin{tabular}{|c|c|c|c|c|}
\hline Name & Expression & Unit & Description & Selection \\
\hline chds2.Dxy_O2 & 0 & $\mathrm{~m}^{\wedge} 2 / \mathrm{s}$ & $\begin{array}{l}\text { Diffusion } \\
\text { coefficient, } x y \\
\text { component }\end{array}$ & Domain 2 \\
\hline chds2.Dyy_O2 & D_O2 & $\mathrm{m}^{\wedge} 2 / \mathrm{s}$ & $\begin{array}{l}\text { Diffusion } \\
\text { coefficient, yy } \\
\text { component }\end{array}$ & Domain 2 \\
\hline chds2.Dzy_O2 & 0 & $\mathrm{~m}^{\wedge} 2 / \mathrm{s}$ & $\begin{array}{l}\text { Diffusion } \\
\text { coefficient, zy } \\
\text { component }\end{array}$ & Domain 2 \\
\hline chds2.Dxz_O2 & 0 & $\mathrm{~m}^{\wedge} 2 / \mathrm{s}$ & $\begin{array}{l}\text { Diffusion } \\
\text { coefficient, } \mathrm{xz} \\
\text { component }\end{array}$ & Domain 2 \\
\hline chds2.Dyz_O2 & 0 & $\mathrm{~m}^{\wedge} 2 / \mathrm{s}$ & $\begin{array}{l}\text { Diffusion } \\
\text { coefficient, yz } \\
\text { component }\end{array}$ & Domain 2 \\
\hline chds2.Dzz_O2 & D_O2 & $\mathrm{m}^{\wedge} 2 / \mathrm{s}$ & $\begin{array}{l}\text { Diffusion } \\
\text { coefficient, } \quad \mathrm{zz} \\
\text { component }\end{array}$ & Domain 2 \\
\hline chds2.Dav_O2 & chds2.Dxx_O2 & $\mathrm{m}^{\wedge} 2 / \mathrm{s}$ & $\begin{array}{l}\text { Average } \\
\text { diffusion } \\
\text { coefficient }\end{array}$ & Domain 2 \\
\hline chds2.tfluxx_O2 & $\begin{array}{l}\text { - } \\
\text { chds2.Dxx_O2*O2x+chds } \\
\text { 2.cfluxx_O2 }\end{array}$ & $\mathrm{mol} /\left(\mathrm{m}^{\wedge} 2^{*} \mathrm{~s}\right)$ & $\begin{array}{l}\text { Total flux, } \mathrm{x} \\
\text { component }\end{array}$ & Domain 2 \\
\hline chds2.tfluxy_O2 & $\begin{array}{l}\text { - } \\
\text { chds2.Dyx_O2*O2x+chds } \\
\text { 2.cfluxy_O2 }\end{array}$ & $\mathrm{mol} /\left(\mathrm{m}^{\wedge} 2^{*} \mathrm{~s}\right)$ & $\begin{array}{l}\text { Total flux, y } \\
\text { component }\end{array}$ & Domain 2 \\
\hline chds2.tfluxz_O2 & $\begin{array}{l}\text { - } \\
\text { chds2.Dzx_O2*O2x+chds } \\
\text { 2.cfluxz_O2 }\end{array}$ & $\mathrm{mol} /\left(\mathrm{m}^{\wedge} 2^{*} \mathrm{~s}\right)$ & $\begin{array}{l}\text { Total flux, } \mathrm{z} \\
\text { component }\end{array}$ & Domain 2 \\
\hline
\end{tabular}




\begin{tabular}{|c|c|c|c|c|}
\hline Name & Expression & Unit & Description & Selection \\
\hline $\begin{array}{l}\text { chds2.dfluxx_O } \\
2\end{array}$ & -chds2.Dxx_O2*O2x & $\mathrm{mol} /\left(\mathrm{m}^{\wedge} 2^{*} \mathrm{~s}\right)$ & $\begin{array}{l}\text { Diffusive flux, } \\
\text { x component }\end{array}$ & Domain 2 \\
\hline $\begin{array}{l}\text { chds2.dfluxy_O } \\
2\end{array}$ & -chds2.Dyx_O2*O2x & $\mathrm{mol} /\left(\mathrm{m}^{\wedge} \mathrm{2}^{*} \mathrm{~s}\right)$ & $\begin{array}{l}\text { Diffusive flux, } \\
\text { y component }\end{array}$ & Domain 2 \\
\hline $\begin{array}{l}\text { chds2.dfluxz_O } \\
2\end{array}$ & -chds2.Dzx_O2*O2x & $\mathrm{mol} /\left(\mathrm{m}^{\wedge} \mathrm{2}^{*} \mathrm{~s}\right)$ & $\begin{array}{l}\text { Diffusive flux, } \\
\text { z component }\end{array}$ & Domain 2 \\
\hline chds2.gradx_O2 & $\mathrm{O} 2 \mathrm{x}$ & $\mathrm{mol} / \mathrm{m}^{\wedge} 4$ & $\begin{array}{l}\text { Concentration } \\
\text { gradient, } \quad \mathrm{X} \\
\text { component }\end{array}$ & Domain 2 \\
\hline chds2.grady_O2 & 0 & $\mathrm{~mol} / \mathrm{m}^{\wedge} 4$ & $\begin{array}{l}\text { Concentration } \\
\text { gradient, } \quad \mathrm{y} \\
\text { component }\end{array}$ & Domain 2 \\
\hline chds2.gradz_O2 & 0 & $\mathrm{~mol} / \mathrm{m}^{\wedge} 4$ & $\begin{array}{l}\text { Concentration } \\
\text { gradient, } \quad \mathrm{Z} \\
\text { component }\end{array}$ & Domain 2 \\
\hline $\begin{array}{l}\text { chds2.dfluxMag } \\
\text { _O2 }\end{array}$ & $\begin{array}{l}\text { sqrt(chds2.dfluxx_O2^2+ } \\
\text { chds2.dfluxy_O2^2+chds } \\
\text { 2.dfluxz_O2^2) }\end{array}$ & $\mathrm{mol} /\left(\mathrm{m}^{\wedge} 2^{*} \mathrm{~s}\right)$ & $\begin{array}{l}\text { Diffusive flux } \\
\text { magnitude }\end{array}$ & Domain 2 \\
\hline $\begin{array}{l}\text { chds2.tfluxMag } \\
\text { _O2 }\end{array}$ & $\begin{array}{l}\text { sqrt(chds2.tfluxx_O2^2+c } \\
\text { hds2.tfluxy_O2^2+chds2.t } \\
\text { fluxz_O2^2) }\end{array}$ & $\mathrm{mol} /\left(\mathrm{m}^{\wedge} 2^{*} \mathrm{~s}\right)$ & $\begin{array}{l}\text { Total flux } \\
\text { magnitude }\end{array}$ & Domain 2 \\
\hline chds2.Dxx_HO2 & D_OH & $\mathrm{m}^{\wedge} 2 / \mathrm{s}$ & $\begin{array}{l}\text { Diffusion } \\
\text { coefficient, } \mathrm{xx} \\
\text { component }\end{array}$ & Domain 2 \\
\hline chds2.Dyx_HO2 & 0 & $\mathrm{~m}^{\wedge} 2 / \mathrm{s}$ & $\begin{array}{l}\text { Diffusion } \\
\text { coefficient, yx } \\
\text { component }\end{array}$ & Domain 2 \\
\hline
\end{tabular}




\begin{tabular}{|c|c|c|c|c|}
\hline Name & Expression & Unit & Description & Selection \\
\hline chds2.Dzx_HO2 & 0 & $\mathrm{~m}^{\wedge} 2 / \mathrm{s}$ & $\begin{array}{l}\text { Diffusion } \\
\text { coefficient, } \mathrm{zx} \\
\text { component }\end{array}$ & Domain 2 \\
\hline chds2.Dxy_HO2 & 0 & $\mathrm{~m}^{\wedge} 2 / \mathrm{s}$ & $\begin{array}{l}\text { Diffusion } \\
\text { coefficient, } x y \\
\text { component }\end{array}$ & Domain 2 \\
\hline chds2.Dyy_HO2 & D_OH & $\mathrm{m}^{\wedge} 2 / \mathrm{s}$ & $\begin{array}{l}\text { Diffusion } \\
\text { coefficient, yy } \\
\text { component }\end{array}$ & Domain 2 \\
\hline chds2.Dzy_HO2 & 0 & $\mathrm{~m}^{\wedge} 2 / \mathrm{s}$ & $\begin{array}{l}\text { Diffusion } \\
\text { coefficient, zy } \\
\text { component }\end{array}$ & Domain 2 \\
\hline chds2.Dxz_HO2 & 0 & $\mathrm{~m}^{\wedge} 2 / \mathrm{s}$ & $\begin{array}{l}\text { Diffusion } \\
\text { coefficient, } \quad \mathrm{xz} \\
\text { component }\end{array}$ & Domain 2 \\
\hline chds2.Dyz_HO2 & 0 & $\mathrm{~m}^{\wedge} 2 / \mathrm{s}$ & $\begin{array}{l}\text { Diffusion } \\
\text { coefficient, yz } \\
\text { component }\end{array}$ & Domain 2 \\
\hline chds2.Dzz_HO2 & D_OH & $\mathrm{m}^{\wedge} 2 / \mathrm{s}$ & $\begin{array}{l}\text { Diffusion } \\
\text { coefficient, } \quad \mathrm{zz} \\
\text { component }\end{array}$ & Domain 2 \\
\hline chds2.Dav_HO2 & chds2.Dxx_HO2 & $\mathrm{m}^{\wedge} 2 / \mathrm{s}$ & $\begin{array}{l}\text { Average } \\
\text { diffusion } \\
\text { coefficient }\end{array}$ & Domain 2 \\
\hline $\begin{array}{l}\text { chds2.tfluxx_H } \\
\text { O2 }\end{array}$ & $\begin{array}{l}\text { - } \\
\text { chds2.Dxx_HO2*HO2x+c } \\
\text { hds2.cfluxx_HO2 }\end{array}$ & $\mathrm{mol} /\left(\mathrm{m}^{\wedge} 2 * \mathrm{~s}\right)$ & $\begin{array}{l}\text { Total flux, } \mathrm{x} \\
\text { component }\end{array}$ & Domain 2 \\
\hline $\begin{array}{l}\text { chds2.tfluxy_H } \\
\text { O2 }\end{array}$ & $\begin{array}{l}\text { - } \\
\text { chds2.Dyx_HO2*HO2x+c } \\
\text { hds2.cfluxy_HO2 }\end{array}$ & $\mathrm{mol} /\left(\mathrm{m}^{\wedge} 2 * \mathrm{~s}\right)$ & $\begin{array}{l}\text { Total flux, y } \\
\text { component }\end{array}$ & Domain 2 \\
\hline
\end{tabular}




\begin{tabular}{|c|c|c|c|c|}
\hline Name & Expression & Unit & Description & Selection \\
\hline $\begin{array}{l}\text { chds2.tfluxz_H } \\
\text { O2 }\end{array}$ & $\begin{array}{l}\text { - } \\
\text { chds2.Dzx_HO2*HO2x+c } \\
\text { hds2.cfluxz_HO2 }\end{array}$ & $\mathrm{mol} /\left(\mathrm{m}^{\wedge} 2^{*} \mathrm{~s}\right)$ & $\begin{array}{l}\text { Total flux, } \mathrm{z} \\
\text { component }\end{array}$ & Domain 2 \\
\hline $\begin{array}{l}\text { chds2.dfluxx_H } \\
\text { O2 }\end{array}$ & -chds2.Dxx_HO2*HO2x & $\mathrm{mol} /\left(\mathrm{m}^{\wedge} 2^{*} \mathrm{~s}\right)$ & $\begin{array}{l}\text { Diffusive flux, } \\
\text { x component }\end{array}$ & Domain 2 \\
\hline $\begin{array}{l}\text { chds2.dfluxy_H } \\
\text { O2 }\end{array}$ & -chds2.Dyx_HO2*HO2x & $\mathrm{mol} /\left(\mathrm{m}^{\wedge} 2^{*} \mathrm{~s}\right)$ & $\begin{array}{l}\text { Diffusive flux, } \\
\text { y component }\end{array}$ & Domain 2 \\
\hline $\begin{array}{l}\text { chds2.dfluxz_H } \\
\text { O2 }\end{array}$ & -chds2.Dzx_HO2*HO2x & $\mathrm{mol} /\left(\mathrm{m}^{\wedge} 2^{*} \mathrm{~s}\right)$ & $\begin{array}{l}\text { Diffusive flux, } \\
\text { z component }\end{array}$ & Domain 2 \\
\hline $\begin{array}{l}\text { chds2.gradx_H } \\
\text { O2 }\end{array}$ & $\mathrm{HO} 2 \mathrm{x}$ & $\mathrm{mol} / \mathrm{m}^{\wedge} 4$ & $\begin{array}{l}\text { Concentration } \\
\text { gradient, } \quad \mathrm{X} \\
\text { component }\end{array}$ & Domain 2 \\
\hline $\begin{array}{l}\text { chds2.grady_H } \\
\text { O2 }\end{array}$ & 0 & $\mathrm{~mol} / \mathrm{m}^{\wedge} 4$ & $\begin{array}{l}\text { Concentration } \\
\text { gradient, y } \\
\text { component }\end{array}$ & Domain 2 \\
\hline $\begin{array}{l}\text { chds2.gradz_HO } \\
2\end{array}$ & 0 & $\mathrm{~mol} / \mathrm{m}^{\wedge} 4$ & $\begin{array}{l}\text { Concentration } \\
\text { gradient, } \quad \mathrm{Z} \\
\text { component }\end{array}$ & Domain 2 \\
\hline $\begin{array}{l}\text { chds2.dfluxMag } \\
\text { _HO2 }\end{array}$ & $\begin{array}{l}\text { sqrt(chds2.dfluxx_HO2^2 } \\
+ \text { +chds2.dfluxy_HO2^2+ch } \\
\text { ds2.dfluxz_HO2^2) }\end{array}$ & $\mathrm{mol} /\left(\mathrm{m}^{\wedge} 2^{*} \mathrm{~s}\right)$ & $\begin{array}{l}\text { Diffusive flux } \\
\text { magnitude }\end{array}$ & Domain 2 \\
\hline $\begin{array}{l}\text { chds2.tfluxMag } \\
\text { _HO2 }\end{array}$ & $\begin{array}{l}\text { sqrt(chds2.tfluxx_HO2^2 } \\
\text { +chds2.tfluxy_HO2^2+ch } \\
\text { ds2.tfluxz_HO2^2) }\end{array}$ & $\mathrm{mol} /\left(\mathrm{m}^{\wedge} 2^{*} \mathrm{~s}\right)$ & $\begin{array}{ll}\text { Total flux } \\
\text { magnitude }\end{array}$ & Domain 2 \\
\hline $\begin{array}{l}\text { chds2.Dxx_HO2 } \\
\text { minus }\end{array}$ & D_OH & $\mathrm{m}^{\wedge} 2 / \mathrm{s}$ & $\begin{array}{l}\text { Diffusion } \\
\text { coefficient, } \mathrm{xx} \\
\text { component }\end{array}$ & Domain 2 \\
\hline
\end{tabular}




\begin{tabular}{|c|c|c|c|c|}
\hline Name & Expression & Unit & Description & Selection \\
\hline $\begin{array}{l}\text { chds2.Dyx_HO2 } \\
\text { minus }\end{array}$ & 0 & $\mathrm{~m}^{\wedge} 2 / \mathrm{s}$ & $\begin{array}{l}\text { Diffusion } \\
\text { coefficient, yx } \\
\text { component }\end{array}$ & Domain 2 \\
\hline $\begin{array}{l}\text { chds2.Dzx_HO2 } \\
\text { minus }\end{array}$ & 0 & $\mathrm{~m}^{\wedge} 2 / \mathrm{s}$ & $\begin{array}{l}\text { Diffusion } \\
\text { coefficient, } \mathrm{zx} \\
\text { component }\end{array}$ & Domain 2 \\
\hline $\begin{array}{l}\text { chds2.Dxy_HO2 } \\
\text { minus }\end{array}$ & 0 & $\mathrm{~m}^{\wedge} 2 / \mathrm{s}$ & $\begin{array}{l}\text { Diffusion } \\
\text { coefficient, } x y \\
\text { component }\end{array}$ & Domain 2 \\
\hline $\begin{array}{l}\text { chds2.Dyy_HO2 } \\
\text { minus }\end{array}$ & D_OH & $\mathrm{m}^{\wedge} 2 / \mathrm{s}$ & $\begin{array}{l}\text { Diffusion } \\
\text { coefficient, yy } \\
\text { component }\end{array}$ & Domain 2 \\
\hline $\begin{array}{l}\text { chds2.Dzy_HO2 } \\
\text { minus }\end{array}$ & 0 & $\mathrm{~m}^{\wedge} 2 / \mathrm{s}$ & $\begin{array}{l}\text { Diffusion } \\
\text { coefficient, zy } \\
\text { component }\end{array}$ & Domain 2 \\
\hline $\begin{array}{l}\text { chds2.Dxz_HO2 } \\
\text { minus }\end{array}$ & 0 & $\mathrm{~m}^{\wedge} 2 / \mathrm{s}$ & $\begin{array}{l}\text { Diffusion } \\
\text { coefficient, } x z \\
\text { component }\end{array}$ & Domain 2 \\
\hline $\begin{array}{l}\text { chds2.Dyz_HO2 } \\
\text { minus }\end{array}$ & 0 & $\mathrm{~m}^{\wedge} 2 / \mathrm{s}$ & $\begin{array}{l}\text { Diffusion } \\
\text { coefficient, yz } \\
\text { component }\end{array}$ & Domain 2 \\
\hline $\begin{array}{l}\text { chds2.Dzz_HO2 } \\
\text { minus }\end{array}$ & D_OH & $\mathrm{m}^{\wedge} 2 / \mathrm{s}$ & $\begin{array}{l}\text { Diffusion } \\
\text { coefficient, } \quad \mathrm{zZ} \\
\text { component }\end{array}$ & Domain 2 \\
\hline $\begin{array}{l}\text { chds2.Dav_HO2 } \\
\text { minus }\end{array}$ & chds2.Dxx_HO2minus & $\mathrm{m}^{\wedge} 2 / \mathrm{s}$ & $\begin{array}{l}\text { Average } \\
\text { diffusion } \\
\text { coefficient }\end{array}$ & Domain 2 \\
\hline $\begin{array}{l}\text { chds2.tfluxx_H } \\
\text { O2minus }\end{array}$ & $\begin{array}{l}\text { - } \\
\text { chds2.Dxx_HO2minus*H }\end{array}$ & $\mathrm{mol} /\left(\mathrm{m}^{\wedge} 2 * \mathrm{~s}\right)$ & $\begin{array}{l}\text { Total flux, } \mathrm{x} \\
\text { component }\end{array}$ & Domain 2 \\
\hline
\end{tabular}




\begin{tabular}{|c|c|c|c|c|}
\hline Name & Expression & Unit & \begin{tabular}{|l|} 
Description \\
\end{tabular} & Selection \\
\hline & $\begin{array}{l}\text { O2minusx+chds2.cfluxx } \\
\text { HO2minus }\end{array}$ & & & \\
\hline $\begin{array}{l}\text { chds2.tfluxy_H } \\
\text { O2minus }\end{array}$ & $\begin{array}{l}\text { - } \\
\text { chds2.Dyx_HO2minus*H } \\
\text { O2minusx+chds2.cfluxy_ } \\
\text { HO2minus }\end{array}$ & $\mathrm{mol} /\left(\mathrm{m}^{\wedge} \mathrm{L}^{*} \mathrm{~s}\right)$ & $\begin{array}{l}\text { Total flux, y } \\
\text { component }\end{array}$ & Domain 2 \\
\hline $\begin{array}{l}\text { chds2.tfluxz_H } \\
\text { O2minus }\end{array}$ & $\begin{array}{l}\text { - } \\
\text { chds2.Dzx_HO2minus*H } \\
\text { O2minusx+chds2.cfluxz_- } \\
\text { HO2minus }\end{array}$ & $\mathrm{mol} /\left(\mathrm{m}^{\wedge} \mathrm{2}^{*} \mathrm{~s}\right)$ & $\begin{array}{l}\text { Total flux, } \mathrm{z} \\
\text { component }\end{array}$ & Domain 2 \\
\hline $\begin{array}{l}\text { chds2.dfluxx_H } \\
\text { O2minus }\end{array}$ & $\begin{array}{l}\text { - } \\
\text { chds2.Dxx_HO2minus*H } \\
\text { O2minusx }\end{array}$ & $\mathrm{mol} /\left(\mathrm{m}^{\wedge} 2 * \mathrm{~s}\right)$ & $\begin{array}{l}\text { Diffusive flux, } \\
\text { x component }\end{array}$ & Domain 2 \\
\hline $\begin{array}{l}\text { chds2.dfluxy_H } \\
\text { O2minus }\end{array}$ & $\begin{array}{l}\text { - } \\
\text { chds2.Dyx_HO2minus*H } \\
\text { O2minusx }\end{array}$ & $\mathrm{mol} /\left(\mathrm{m}^{\wedge} 2 * \mathrm{~s}\right)$ & $\begin{array}{l}\text { Diffusive flux, } \\
\text { y component }\end{array}$ & Domain 2 \\
\hline $\begin{array}{l}\text { chds2.dfluxz_H } \\
\text { O2minus }\end{array}$ & $\begin{array}{l}\text { - } \\
\text { chds2.Dzx_HO2minus*H } \\
\text { O2minusx }\end{array}$ & $\mathrm{mol} /\left(\mathrm{m}^{\wedge} 2^{*} \mathrm{~s}\right)$ & $\begin{array}{l}\text { Diffusive flux, } \\
\text { z component }\end{array}$ & Domain 2 \\
\hline $\begin{array}{l}\text { chds2.gradx_H } \\
\text { O2minus }\end{array}$ & HO2minusx & $\mathrm{mol} / \mathrm{m}^{\wedge} 4$ & $\begin{array}{l}\text { Concentration } \\
\text { gradient, } \quad \mathrm{x} \\
\text { component }\end{array}$ & Domain 2 \\
\hline $\begin{array}{l}\text { chds2.grady_H } \\
\text { O2minus }\end{array}$ & 0 & $\mathrm{~mol} / \mathrm{m}^{\wedge} \wedge$ & $\begin{array}{l}\text { Concentration } \\
\text { gradient, y } \\
\text { component }\end{array}$ & Domain 2 \\
\hline $\begin{array}{l}\text { chds2.gradz_HO } \\
\text { 2minus }\end{array}$ & 0 & $\mathrm{~mol} / \mathrm{m}^{\wedge} 4$ & $\begin{array}{l}\text { Concentration } \\
\text { gradient, } \quad \mathrm{z} \\
\text { component }\end{array}$ & Domain 2 \\
\hline $\begin{array}{l}\text { chds2.dfluxMag } \\
\text { _HO2minus }\end{array}$ & $\begin{array}{l}\text { sqrt(chds2.dfluxx_HO2mi } \\
\text { nus^2+chds2.dfluxy_HO2 }\end{array}$ & $\mathrm{mol} /\left(\mathrm{m}^{\wedge} 2 * \mathrm{~s}\right)$ & $\begin{array}{l}\text { Diffusive flux } \\
\text { magnitude }\end{array}$ & Domain 2 \\
\hline
\end{tabular}




\begin{tabular}{|c|c|c|c|c|}
\hline Name & Expression & Unit & Description & Selection \\
\hline & $\begin{array}{l}\text { minus } \wedge 2+\text { chds2.dfluxz_H } \\
\text { O2minus } \wedge 2 \text { ) }\end{array}$ & & & \\
\hline $\begin{array}{l}\text { chds2.tfluxMag } \\
\text { _HO2minus }\end{array}$ & $\begin{array}{l}\text { sqrt(chds2.tfluxx_HO2mi } \\
\text { nus^2+chds2.tfluxy_HO2 } \\
\text { minus^2+chds2.tfluxz_H } \\
\text { O2minus^2) }\end{array}$ & $\mathrm{mol} /\left(\mathrm{m}^{\wedge} 2^{*} \mathrm{~s}\right)$ & $\begin{array}{l}\text { Total flux } \\
\text { magnitude }\end{array}$ & Domain 2 \\
\hline $\begin{array}{l}\text { chds2.Dxx_H2O } \\
2\end{array}$ & $1.0 \mathrm{E}-9\left[\mathrm{~m}^{\wedge} \mathrm{2} / \mathrm{s}\right]$ & $\mathrm{m}^{\wedge} 2 / \mathrm{s}$ & $\begin{array}{l}\text { Diffusion } \\
\text { coefficient, } \mathrm{xx} \\
\text { component }\end{array}$ & Domain 2 \\
\hline $\begin{array}{l}\text { chds2.Dyx_H2O } \\
2\end{array}$ & 0 & $\mathrm{~m}^{\wedge} 2 / \mathrm{s}$ & $\begin{array}{l}\text { Diffusion } \\
\text { coefficient, yx } \\
\text { component }\end{array}$ & Domain 2 \\
\hline $\begin{array}{l}\text { chds2.Dzx_H2O } \\
2\end{array}$ & 0 & $\mathrm{~m}^{\wedge} 2 / \mathrm{s}$ & $\begin{array}{l}\text { Diffusion } \\
\text { coefficient, zx } \\
\text { component }\end{array}$ & Domain 2 \\
\hline $\begin{array}{l}\text { chds2.Dxy_H2O } \\
2\end{array}$ & 0 & $\mathrm{~m}^{\wedge} 2 / \mathrm{s}$ & $\begin{array}{l}\text { Diffusion } \\
\text { coefficient, } \quad x y \\
\text { component }\end{array}$ & Domain 2 \\
\hline $\begin{array}{l}\text { chds2.Dyy_H2O } \\
2\end{array}$ & $1.0 \mathrm{E}-9\left[\mathrm{~m}^{\wedge} \mathrm{2} / \mathrm{s}\right]$ & $\mathrm{m}^{\wedge} 2 / \mathrm{s}$ & $\begin{array}{l}\text { Diffusion } \\
\text { coefficient, yy } \\
\text { component }\end{array}$ & Domain 2 \\
\hline $\begin{array}{l}\text { chds2.Dzy_H2O } \\
2\end{array}$ & 0 & $\mathrm{~m}^{\wedge} 2 / \mathrm{s}$ & $\begin{array}{l}\text { Diffusion } \\
\text { coefficient, zy } \\
\text { component }\end{array}$ & Domain 2 \\
\hline $\begin{array}{l}\text { chds2.Dxz_H2O } \\
2\end{array}$ & 0 & $\mathrm{~m}^{\wedge} 2 / \mathrm{s}$ & $\begin{array}{l}\text { Diffusion } \\
\text { coefficient, } \quad \mathrm{xz} \\
\text { component }\end{array}$ & Domain 2 \\
\hline $\begin{array}{l}\text { chds2.Dyz_H2O } \\
2\end{array}$ & 0 & $\mathrm{~m}^{\wedge} 2 / \mathrm{s}$ & $\begin{array}{l}\text { Diffusion } \\
\text { coefficient, yz } \\
\text { component }\end{array}$ & Domain 2 \\
\hline
\end{tabular}




\begin{tabular}{|c|c|c|c|c|}
\hline Name & Expression & Unit & Description & Selection \\
\hline $\begin{array}{l}\text { chds2.Dzz_H2O } \\
2\end{array}$ & $1.0 \mathrm{E}-9\left[\mathrm{~m}^{\wedge} 2 / \mathrm{s}\right]$ & $\mathrm{m}^{\wedge} 2 / \mathrm{s}$ & $\begin{array}{l}\text { Diffusion } \\
\text { coefficient, } \quad \mathrm{zz} \\
\text { component }\end{array}$ & Domain 2 \\
\hline $\begin{array}{l}\text { chds2.Dav_H2O } \\
2\end{array}$ & chds2.Dxx_H2O2 & $\mathrm{m}^{\wedge} 2 / \mathrm{s}$ & $\begin{array}{l}\text { Average } \\
\text { diffusion } \\
\text { coefficient }\end{array}$ & Domain 2 \\
\hline $\begin{array}{l}\text { chds2.tfluxx_H2 } \\
\text { O2 }\end{array}$ & $\begin{array}{l}\text { - } \\
\text { chds2.Dxx_H2O2*H2O2x } \\
\text { +chds2.cfluxx_H2O2 }\end{array}$ & $\mathrm{mol} /\left(\mathrm{m}^{\wedge} 2^{*} \mathrm{~s}\right)$ & $\begin{array}{l}\text { Total flux, } \mathrm{x} \\
\text { component }\end{array}$ & Domain 2 \\
\hline $\begin{array}{l}\text { chds2.tfluxy_H2 } \\
\text { O2 }\end{array}$ & $\begin{array}{l}- \\
\text { chds2.Dyx_H2O2*H2O2x } \\
\text { +chds2.cfluxy_H2O2 }\end{array}$ & $\mathrm{mol} /\left(\mathrm{m}^{\wedge} 2^{*} \mathrm{~s}\right)$ & $\begin{array}{l}\text { Total flux, y } \\
\text { component }\end{array}$ & Domain 2 \\
\hline $\begin{array}{l}\text { chds2.tfluxz_H2 } \\
\text { O2 }\end{array}$ & $\begin{array}{l}- \\
\text { chds2.Dzx_H2O2*H2O2x } \\
\text { +chds2.cfluxz_H2O2 }\end{array}$ & $\mathrm{mol} /\left(\mathrm{m}^{\wedge} 2^{*} \mathrm{~s}\right)$ & $\begin{array}{l}\text { Total flux, } \mathrm{z} \\
\text { component }\end{array}$ & Domain 2 \\
\hline $\begin{array}{l}\text { chds2.dfluxx_H } \\
2 \mathrm{O} 2\end{array}$ & $\begin{array}{l}\text { - } \\
\text { chds2.Dxx_H2O2*H2O2x }\end{array}$ & $\mathrm{mol} /\left(\mathrm{m}^{\wedge} 2^{*} \mathrm{~s}\right)$ & $\begin{array}{l}\text { Diffusive flux, } \\
\text { x component }\end{array}$ & Domain 2 \\
\hline $\begin{array}{l}\text { chds2.dfluxy_H } \\
2 \mathrm{O} 2\end{array}$ & $\begin{array}{l}- \\
\text { chds2.Dyx_H2O2*H2O2x }\end{array}$ & $\mathrm{mol} /\left(\mathrm{m}^{\wedge} 2^{*} \mathrm{~s}\right)$ & $\begin{array}{l}\text { Diffusive flux, } \\
\text { y component }\end{array}$ & Domain 2 \\
\hline $\begin{array}{l}\text { chds2.dfluxz_H } \\
2 \mathrm{O} 2\end{array}$ & $\begin{array}{l}- \\
\text { chds2.Dzx_H2O2*H2O2x }\end{array}$ & $\mathrm{mol} /\left(\mathrm{m}^{\wedge} 2^{*} \mathrm{~s}\right)$ & $\begin{array}{l}\text { Diffusive flux, } \\
\text { z component }\end{array}$ & Domain 2 \\
\hline $\begin{array}{l}\text { chds2.gradx_H2 } \\
\text { O2 }\end{array}$ & $\mathrm{H} 2 \mathrm{O} 2 \mathrm{x}$ & $\mathrm{mol} / \mathrm{m}^{\wedge} 4$ & $\begin{array}{l}\text { Concentration } \\
\text { gradient, } \quad \mathrm{x} \\
\text { component }\end{array}$ & Domain 2 \\
\hline $\begin{array}{l}\text { chds2.grady_H2 } \\
\text { O2 }\end{array}$ & 0 & $\mathrm{~mol} / \mathrm{m}^{\wedge} 4$ & $\begin{array}{l}\text { Concentration } \\
\text { gradient, y } \\
\text { component }\end{array}$ & Domain 2 \\
\hline
\end{tabular}




\begin{tabular}{|c|c|c|c|c|}
\hline Name & Expression & Unit & Description & Selection \\
\hline $\begin{array}{l}\text { chds2.gradz_H2 } \\
\text { O2 }\end{array}$ & 0 & $\mathrm{~mol} / \mathrm{m}^{\wedge} 4$ & $\begin{array}{l}\text { Concentration } \\
\text { gradient, } \quad \mathrm{Z} \\
\text { component }\end{array}$ & Domain 2 \\
\hline $\begin{array}{l}\text { chds2.dfluxMag } \\
\text { _H2O2 }\end{array}$ & $\begin{array}{l}\text { sqrt(chds2.dfluxx_H2O2^ } \\
\text { 2+chds2.dfluxy_H2O2^2 } \\
\text { +chds2.dfluxz_H2O2^2) }\end{array}$ & $\mathrm{mol} /\left(\mathrm{m}^{\wedge} 2^{*} \mathrm{~s}\right)$ & $\begin{array}{l}\text { Diffusive flux } \\
\text { magnitude }\end{array}$ & Domain 2 \\
\hline $\begin{array}{l}\text { chds2.tfluxMag } \\
\text { _H2O2 }\end{array}$ & $\begin{array}{l}\text { sqrt(chds2.tfluxx_H2O2^2 } \\
\text { +chds2.tfluxy_H2O2^2+c } \\
\text { hds2.tfluxz_H2O2^2) }\end{array}$ & $\mathrm{mol} /\left(\mathrm{m}^{\wedge} 2^{*} \mathrm{~s}\right)$ & $\begin{array}{l}\text { Total flux } \\
\text { magnitude }\end{array}$ & Domain 2 \\
\hline chds2.u & model.input.u1 & $\mathrm{m} / \mathrm{s}$ & $\begin{array}{l}\text { Velocity field, } \\
\text { x component }\end{array}$ & Domain 2 \\
\hline chds2.v & model.input.u2 & $\mathrm{m} / \mathrm{s}$ & $\begin{array}{l}\text { Velocity field, } \\
\text { y component }\end{array}$ & Domain 2 \\
\hline chds2.w & model.input.u3 & $\mathrm{m} / \mathrm{s}$ & $\begin{array}{l}\text { Velocity field, } \\
\text { z component }\end{array}$ & Domain 2 \\
\hline $\begin{array}{l}\text { chds2.cfluxx_M } \\
\text { plus }\end{array}$ & Mplus*model.input.u1 & $\mathrm{mol} /\left(\mathrm{m}^{\wedge} 2^{*} \mathrm{~s}\right)$ & $\begin{array}{l}\text { Convective } \\
\text { flux, } \quad \mathrm{x} \\
\text { component }\end{array}$ & Domain 2 \\
\hline $\begin{array}{l}\text { chds2.cfluxy_M } \\
\text { plus }\end{array}$ & Mplus*model.input.u2 & $\mathrm{mol} /\left(\mathrm{m}^{\wedge} 2^{*} \mathrm{~s}\right)$ & $\begin{array}{l}\text { Convective } \\
\text { flux, } \quad \mathrm{y} \\
\text { component }\end{array}$ & Domain 2 \\
\hline $\begin{array}{l}\text { chds2.cfluxz_M } \\
\text { plus }\end{array}$ & Mplus*model.input.u3 & $\mathrm{mol} /\left(\mathrm{m}^{\wedge} 2 * \mathrm{~s}\right)$ & $\begin{array}{l}\text { Convective } \\
\text { flux, } \quad \mathrm{Z} \\
\text { component }\end{array}$ & Domain 2 \\
\hline $\begin{array}{l}\text { chds2.cfluxMag } \\
\text { _Mplus }\end{array}$ & $\begin{array}{l}\text { sqrt(chds2.cfluxx_Mplus^ } \\
\text { 2+chds2.cfluxy_Mplus^2 } \\
\text { +chds2.cfluxz_Mplus^2) }\end{array}$ & $\mathrm{mol} /\left(\mathrm{m}^{\wedge} 2^{*} \mathrm{~s}\right)$ & $\begin{array}{l}\text { Convective } \\
\text { flux magnitude }\end{array}$ & Domain 2 \\
\hline
\end{tabular}




\begin{tabular}{|c|c|c|c|c|}
\hline Name & Expression & Unit & Description & Selection \\
\hline $\begin{array}{l}\text { chds2.cfluxx_O } \\
\text { H }\end{array}$ & $\mathrm{OH}^{*}$ model.input.u1 & $\mathrm{mol} /\left(\mathrm{m}^{\wedge} 2 * \mathrm{~s}\right)$ & $\begin{array}{l}\text { Convective } \\
\text { flux, } \quad \mathrm{x} \\
\text { component }\end{array}$ & Domain 2 \\
\hline $\begin{array}{l}\text { chds2.cfluxy_O } \\
\text { H }\end{array}$ & OH*model.input.u2 & $\mathrm{mol} /\left(\mathrm{m}^{\wedge} 2 * \mathrm{~s}\right)$ & $\begin{array}{l}\text { Convective } \\
\text { flux, } \quad y \\
\text { component }\end{array}$ & Domain 2 \\
\hline $\begin{array}{l}\text { chds2.cfluxz_O } \\
\text { H }\end{array}$ & OH*model.input.u3 & $\mathrm{mol} /\left(\mathrm{m}^{\wedge} 2 * \mathrm{~s}\right)$ & $\begin{array}{l}\text { Convective } \\
\text { flux, } \quad \mathrm{z} \\
\text { component }\end{array}$ & Domain 2 \\
\hline $\begin{array}{l}\text { chds2.cfluxMag } \\
\text { _OH }\end{array}$ & $\begin{array}{l}\text { sqrt(chds2.cfluxx_OH^2+ } \\
\text { chds2.cfluxy_OH^2+chds } \\
\text { 2.cfluxz_OH^2) }\end{array}$ & $\mathrm{mol} /\left(\mathrm{m}^{\wedge} 2 * \mathrm{~s}\right)$ & $\begin{array}{l}\text { Convective } \\
\text { flux magnitude }\end{array}$ & Domain 2 \\
\hline chds2.cfluxx_Fc & Fc*model.input.u1 & $\mathrm{mol} /\left(\mathrm{m}^{\wedge} 2 * \mathrm{~s}\right)$ & $\begin{array}{l}\text { Convective } \\
\text { flux, } \quad \mathrm{x} \\
\text { component }\end{array}$ & Domain 2 \\
\hline chds2.cfluxy_Fc & Fc*model.input.u2 & $\mathrm{mol} /\left(\mathrm{m}^{\wedge} 2 * \mathrm{~s}\right)$ & $\begin{array}{l}\text { Convective } \\
\text { flux, } \quad y \\
\text { component }\end{array}$ & Domain 2 \\
\hline chds2.cfluxz_Fc & Fc*model.input.u3 & $\mathrm{mol} /\left(\mathrm{m}^{\wedge} 2 * \mathrm{~s}\right)$ & $\begin{array}{l}\text { Convective } \\
\text { flux, } \quad \mathrm{z} \\
\text { component }\end{array}$ & Domain 2 \\
\hline $\begin{array}{l}\text { chds2.cfluxMag } \\
\text { _Fc }\end{array}$ & $\begin{array}{l}\text { sqrt(chds2.cfluxx_Fc^2+c } \\
\text { hds2.cfluxy_Fc^2+chds2. } \\
\text { cfluxz_Fc^2) }\end{array}$ & $\mathrm{mol} /\left(\mathrm{m}^{\wedge} 2^{*} \mathrm{~s}\right)$ & $\begin{array}{l}\text { Convective } \\
\text { flux magnitude }\end{array}$ & Domain 2 \\
\hline $\begin{array}{l}\text { chds2.cfluxx_Fc } \\
\text { H }\end{array}$ & FcH*model.input.u1 & $\mathrm{mol} /\left(\mathrm{m}^{\wedge} 2 * \mathrm{~s}\right)$ & $\begin{array}{ll}\text { Convective } & \\
\text { flux, } & \mathrm{x} \\
\text { component } & \end{array}$ & Domain 2 \\
\hline $\begin{array}{l}\text { chds2.cfluxy_Fc } \\
\text { H }\end{array}$ & FcH*model.input.u2 & $\mathrm{mol} /\left(\mathrm{m}^{\wedge} 2 * \mathrm{~s}\right)$ & $\begin{array}{l}\text { Convective } \\
\text { flux, } \quad \mathrm{y} \\
\text { component }\end{array}$ & Domain 2 \\
\hline
\end{tabular}




\begin{tabular}{|c|c|c|c|c|}
\hline Name & Expression & Unit & Description & Selection \\
\hline $\begin{array}{l}\text { chds2.cfluxz_Fc } \\
\mathrm{H}\end{array}$ & FcH*model.input.u3 & $\mathrm{mol} /\left(\mathrm{m}^{\wedge} 2 * \mathrm{~s}\right)$ & $\begin{array}{l}\text { Convective } \\
\text { flux, } \quad \mathrm{z} \\
\text { component }\end{array}$ & Domain 2 \\
\hline $\begin{array}{l}\text { chds2.cfluxMag } \\
\text { _FcH }\end{array}$ & $\begin{array}{l}\text { sqrt(chds2.cfluxx_FcH } 2 \\
+ \text { chds2.cfluxy_FcH^2+ch } \\
\text { ds2.cfluxz_FcH^2) }\end{array}$ & $\mathrm{mol} /\left(\mathrm{m}^{\wedge} 2 * \mathrm{~s}\right)$ & $\begin{array}{l}\text { Convective } \\
\text { flux magnitude }\end{array}$ & Domain 2 \\
\hline $\begin{array}{l}\text { chds2.cfluxx_Fc } \\
\text { plus }\end{array}$ & Fcplus*model.input.u1 & $\mathrm{mol} /\left(\mathrm{m}^{\wedge} 2 * \mathrm{~s}\right)$ & $\begin{array}{ll}\text { Convective } & \\
\text { flux, } & \mathrm{x} \\
\text { component } & \end{array}$ & Domain 2 \\
\hline $\begin{array}{l}\text { chds2.cfluxy_Fc } \\
\text { plus }\end{array}$ & Fcplus*model.input.u2 & $\mathrm{mol} /\left(\mathrm{m}^{\wedge} 2 * \mathrm{~s}\right)$ & $\begin{array}{l}\text { Convective } \\
\text { flux, } \quad y \\
\text { component }\end{array}$ & Domain 2 \\
\hline $\begin{array}{l}\text { chds2.cfluxz_Fc } \\
\text { plus }\end{array}$ & Fcplus*model.input.u3 & $\mathrm{mol} /\left(\mathrm{m}^{\wedge} 2 * \mathrm{~s}\right)$ & $\begin{array}{l}\text { Convective } \\
\text { flux, } \quad \mathrm{z} \\
\text { component }\end{array}$ & Domain 2 \\
\hline $\begin{array}{l}\text { chds2.cfluxMag } \\
\text { _Fcplus }\end{array}$ & $\begin{array}{l}\text { sqrt(chds2.cfluxx_Fcplus }{ }^{\wedge} \\
\text { 2+chds2.cfluxy_Fcplus^2 } \\
\text { +chds2.cfluxz_Fcplus^2) }\end{array}$ & $\mathrm{mol} /\left(\mathrm{m}^{\wedge} 2 * \mathrm{~s}\right)$ & $\begin{array}{l}\text { Convective } \\
\text { flux magnitude }\end{array}$ & Domain 2 \\
\hline $\begin{array}{l}\text { chds2.cfluxx_M } \\
\text { OHorg }\end{array}$ & MOHorg*model.input.u1 & $\mathrm{mol} /\left(\mathrm{m}^{\wedge} 2 * \mathrm{~s}\right)$ & $\begin{array}{ll}\text { Convective } & \\
\text { flux, } & \mathrm{x} \\
\text { component } & \end{array}$ & Domain 2 \\
\hline $\begin{array}{l}\text { chds2.cfluxy_M } \\
\text { OHorg }\end{array}$ & MOHorg*model.input.u2 & $\mathrm{mol} /\left(\mathrm{m}^{\wedge} 2^{*} \mathrm{~s}\right)$ & $\begin{array}{ll}\text { Convective } & \\
\text { flux, } \quad y \\
\text { component }\end{array}$ & Domain 2 \\
\hline $\begin{array}{l}\text { chds2.cfluxz_M } \\
\text { OHorg }\end{array}$ & MOHorg*model.input.u3 & $\mathrm{mol} /\left(\mathrm{m}^{\wedge} 2 * \mathrm{~s}\right)$ & $\begin{array}{l}\text { Convective } \\
\text { flux, } \quad \mathrm{z} \\
\text { component }\end{array}$ & Domain 2 \\
\hline $\begin{array}{l}\text { chds2.cfluxMag } \\
\text { _MOHorg }\end{array}$ & $\begin{array}{l}\text { sqrt(chds2.cfluxx_MOHor } \\
\mathrm{g}^{\wedge} 2+\text { chds2.cfluxy_MOHo }\end{array}$ & $\mathrm{mol} /\left(\mathrm{m}^{\wedge} 2 * \mathrm{~s}\right)$ & $\begin{array}{l}\text { Convective } \\
\text { flux magnitude }\end{array}$ & Domain 2 \\
\hline
\end{tabular}




\begin{tabular}{|c|c|c|c|c|}
\hline Name & Expression & Unit & Description & Selection \\
\hline & $\begin{array}{l}\text { rg^2+chds2.cfluxz_MOH } \\
\operatorname{org} \wedge 2)\end{array}$ & & & \\
\hline $\begin{array}{l}\text { chds2.cfluxx_O } \\
2\end{array}$ & O2*model.input.u1 & $\mathrm{mol} /\left(\mathrm{m}^{\wedge} 2 * \mathrm{~s}\right)$ & $\begin{array}{l}\text { Convective } \\
\text { flux, } \quad \mathrm{x} \\
\text { component }\end{array}$ & Domain 2 \\
\hline $\begin{array}{l}\text { chds2.cfluxy_O } \\
2\end{array}$ & O2*model.input.u2 & $\mathrm{mol} /\left(\mathrm{m}^{\wedge} 2 * \mathrm{~s}\right)$ & $\begin{array}{ll}\text { Convective } & \\
\text { flux, } \quad y \\
\text { component }\end{array}$ & Domain 2 \\
\hline $\begin{array}{l}\text { chds2.cfluxz_O } \\
2\end{array}$ & O2*model.input.u3 & $\mathrm{mol} /\left(\mathrm{m}^{\wedge} 2 * \mathrm{~s}\right)$ & $\begin{array}{l}\text { Convective } \\
\text { flux, } \quad \mathrm{Z} \\
\text { component }\end{array}$ & Domain 2 \\
\hline $\begin{array}{l}\text { chds2.cfluxMag } \\
\text { _O2 }\end{array}$ & $\begin{array}{l}\text { sqrt(chds2.cfluxx_O2^2+c } \\
\text { hds2.cfluxy_O2^2+chds2. } \\
\text { cfluxz_O2^2) }\end{array}$ & $\mathrm{mol} /\left(\mathrm{m}^{\wedge} 2 * \mathrm{~s}\right)$ & $\begin{array}{l}\text { Convective } \\
\text { flux magnitude }\end{array}$ & Domain 2 \\
\hline $\begin{array}{l}\text { chds2.cfluxx_H } \\
\text { O2 }\end{array}$ & HO2*model.input.u1 & $\mathrm{mol} /\left(\mathrm{m}^{\wedge} 2^{*} \mathrm{~s}\right)$ & $\begin{array}{l}\text { Convective } \\
\text { flux, } \quad \mathrm{x} \\
\text { component }\end{array}$ & Domain 2 \\
\hline $\begin{array}{l}\text { chds2.cfluxy_H } \\
\text { O2 }\end{array}$ & HO2*model.input.u2 & $\mathrm{mol} /\left(\mathrm{m}^{\wedge} 2 * \mathrm{~s}\right)$ & $\begin{array}{l}\text { Convective } \\
\text { flux, } \quad y \\
\text { component }\end{array}$ & Domain 2 \\
\hline $\begin{array}{l}\text { chds2.cfluxz_H } \\
\text { O2 }\end{array}$ & HO2*model.input.u3 & $\mathrm{mol} /\left(\mathrm{m}^{\wedge} 2^{*} \mathrm{~s}\right)$ & $\begin{array}{l}\text { Convective } \\
\text { flux, } \quad \mathrm{z} \\
\text { component }\end{array}$ & Domain 2 \\
\hline $\begin{array}{l}\text { chds2.cfluxMag } \\
\text { _HO2 }\end{array}$ & $\begin{array}{l}\text { sqrt(chds2.cfluxx_HO2^2 } \\
\text { +chds2.cfluxy_HO2^2+ch } \\
\text { ds2.cfluxz_HO2^2) }\end{array}$ & $\mathrm{mol} /\left(\mathrm{m}^{\wedge} 2 * \mathrm{~s}\right)$ & $\begin{array}{l}\text { Convective } \\
\text { flux magnitude }\end{array}$ & Domain 2 \\
\hline $\begin{array}{l}\text { chds2.cfluxx_H } \\
\text { O2minus }\end{array}$ & $\begin{array}{l}\text { HO2minus*model.input.u } \\
1\end{array}$ & $\mathrm{~mol} /\left(\mathrm{m}^{\wedge} 2 * \mathrm{~s}\right)$ & $\begin{array}{ll}\text { Convective } & \\
\text { flux, } & \mathrm{x} \\
\text { component } & \end{array}$ & Domain 2 \\
\hline
\end{tabular}




\begin{tabular}{|c|c|c|c|c|}
\hline Name & Expression & Unit & Description & Selection \\
\hline $\begin{array}{l}\text { chds2.cfluxy_H } \\
\text { O2minus }\end{array}$ & $\begin{array}{l}\text { HO2minus*model.input.u } \\
2\end{array}$ & $\mathrm{~mol} /\left(\mathrm{m}^{\wedge} 2 * \mathrm{~s}\right)$ & $\begin{array}{l}\text { Convective } \\
\text { flux, } \quad \mathrm{y} \\
\text { component }\end{array}$ & Domain 2 \\
\hline $\begin{array}{l}\text { chds2.cfluxz_H } \\
\text { O2minus }\end{array}$ & $\begin{array}{l}\text { HO2minus*model.input.u } \\
3\end{array}$ & $\mathrm{~mol} /\left(\mathrm{m}^{\wedge} 2^{*} \mathrm{~s}\right)$ & $\begin{array}{l}\text { Convective } \\
\text { flux, } \quad \mathrm{Z} \\
\text { component }\end{array}$ & Domain 2 \\
\hline $\begin{array}{l}\text { chds2.cfluxMag } \\
\text { _HO2minus }\end{array}$ & $\begin{array}{l}\text { sqrt(chds2.cfluxx_HO2mi } \\
\text { nus^2+chds2.cfluxy_HO2 } \\
\text { minus^2+chds2.cfluxz_H } \\
\text { O2minus^2) }\end{array}$ & $\mathrm{mol} /\left(\mathrm{m}^{\wedge} 2 * \mathrm{~s}\right)$ & $\begin{array}{l}\text { Convective } \\
\text { flux magnitude }\end{array}$ & Domain 2 \\
\hline $\begin{array}{l}\text { chds2.cfluxx_H } \\
2 \mathrm{O} 2\end{array}$ & H2O2*model.input.u1 & $\mathrm{mol} /\left(\mathrm{m}^{\wedge} 2 * \mathrm{~s}\right)$ & $\begin{array}{l}\text { Convective } \\
\text { flux, } \quad \mathrm{x} \\
\text { component }\end{array}$ & Domain 2 \\
\hline $\begin{array}{l}\text { chds2.cfluxy_H } \\
2 \mathrm{O} 2\end{array}$ & H2O2*model.input.u2 & $\mathrm{mol} /\left(\mathrm{m}^{\wedge} 2 * \mathrm{~s}\right)$ & $\begin{array}{ll}\text { Convective } & \\
\text { flux, } \quad y \\
\text { component }\end{array}$ & Domain 2 \\
\hline $\begin{array}{l}\text { chds2.cfluxz_H } \\
2 \mathrm{O} 2\end{array}$ & H2O2*model.input.u3 & $\mathrm{mol} /\left(\mathrm{m}^{\wedge} 2^{*} \mathrm{~s}\right)$ & $\begin{array}{l}\text { Convective } \\
\text { flux, } \quad \mathrm{z} \\
\text { component }\end{array}$ & Domain 2 \\
\hline $\begin{array}{l}\text { chds2.cfluxMag } \\
\text { _H2O2 }\end{array}$ & $\begin{array}{l}\text { sqrt(chds2.cfluxx_H2O2^ } \\
\text { 2+chds2.cfluxy_H2O2^2+ } \\
\text { chds2.cfluxz_H2O2^2) }\end{array}$ & $\mathrm{mol} /\left(\mathrm{m}^{\wedge} 2 * \mathrm{~s}\right)$ & $\begin{array}{l}\text { Convective } \\
\text { flux magnitude }\end{array}$ & Domain 2 \\
\hline chds2.helem & $\mathrm{h}$ & $\mathrm{m}$ & Element size & Domain 2 \\
\hline $\begin{array}{l}\text { chds2.Res_Mplu } \\
\text { s }\end{array}$ & $\begin{array}{l}\text { chds2.u*Mplusx- } \\
\text { chds2.R_Mplus }\end{array}$ & $\mathrm{mol} /\left(\mathrm{m}^{\wedge} 3^{*} \mathrm{~s}\right)$ & $\begin{array}{l}\text { Equation } \\
\text { residual }\end{array}$ & Domain 2 \\
\hline chds2.Res_OH & $\begin{array}{l}\text { chds2.u*OHx- } \\
\text { chds2.R_OH }\end{array}$ & $\mathrm{mol} /\left(\mathrm{m}^{\wedge} 3^{*} \mathrm{~s}\right)$ & $\begin{array}{l}\text { Equation } \\
\text { residual }\end{array}$ & Domain 2 \\
\hline chds2.Res_Fc & chds2.u*Fcx-chds2.R_Fc & $\mathrm{mol} /\left(\mathrm{m}^{\wedge} 3^{*} \mathrm{~s}\right)$ & $\begin{array}{l}\text { Equation } \\
\text { residual }\end{array}$ & Domain 2 \\
\hline
\end{tabular}




\begin{tabular}{|l|l|l|l|l|}
\hline Name & Expression & Unit & Description & Selection \\
\hline chds2.Res_FcH & $\begin{array}{l}\text { chds2.u*FcHx- } \\
\text { chds2.R_FcH }\end{array}$ & $\mathrm{mol} /\left(\mathrm{m}^{\wedge} 3^{*} \mathrm{~s}\right)$ & $\begin{array}{l}\text { Equation } \\
\text { residual }\end{array}$ & Domain 2 \\
\hline $\begin{array}{l}\text { chds2.Res_Fcpl } \\
\text { us }\end{array}$ & $\begin{array}{l}\text { chds2.u*Fcplusx- } \\
\text { chds2.R_Fcplus }\end{array}$ & $\mathrm{mol} /\left(\mathrm{m}^{\wedge} 3^{*} \mathrm{~s}\right)$ & $\begin{array}{l}\text { Equation } \\
\text { residual }\end{array}$ & Domain 2 \\
\hline $\begin{array}{l}\text { chds2.Res_MO } \\
\text { Horg }\end{array}$ & $\begin{array}{l}\text { chds2.u*MOHorgx- } \\
\text { chds2.R_MOHorg }\end{array}$ & $\mathrm{mol} /\left(\mathrm{m}^{\wedge} 3^{*} \mathrm{~s}\right)$ & $\begin{array}{l}\text { Equation } \\
\text { residual }\end{array}$ & Domain 2 \\
\hline chds2.Res_O2 & $\begin{array}{l}\text { chds2.u*O2x-chds2.R_O2 } \\
\mathrm{mol} /\left(\mathrm{m}^{\wedge} 3^{*} \mathrm{~s}\right)\end{array}$ & $\begin{array}{l}\text { Equation } \\
\text { residual }\end{array}$ & Domain 2 \\
\hline chds2.Res_HO2 & $\begin{array}{l}\text { chds2.u*HO2x- } \\
\text { chds2.R_HO2 }\end{array}$ & $\mathrm{mol} /\left(\mathrm{m}^{\wedge} 3^{*} \mathrm{~s}\right)$ & $\begin{array}{l}\text { Equation } \\
\text { residual }\end{array}$ & Domain 2 \\
\hline chds2.Res_HO2 & $\begin{array}{l}\text { chds2.u*HO2minusx- } \\
\text { minus }\end{array}$ & $\begin{array}{l}\mathrm{mol} /\left(\mathrm{m}^{\wedge} 3^{*} \mathrm{~s}\right) \\
\text { chds2.R_HO2minus }\end{array}$ & $\begin{array}{l}\text { Equation } \\
\text { residual }\end{array}$ & Domain 2 \\
\hline chds2.Res_H2O & $\begin{array}{l}\text { chds2.u*H2O2x- } \\
\text { chds2.R_H2O2 }\end{array}$ & $\mathrm{mol} /\left(\mathrm{m}^{\wedge} 3^{*} \mathrm{~s}\right)$ & $\begin{array}{l}\text { Equation } \\
\text { residual }\end{array}$ & Domain 2 \\
\hline
\end{tabular}

Shape functions

\begin{tabular}{|l|l|l|l|l|l|}
\hline Name & Shape function & Unit & Description & Shape frame & Selection \\
\hline Mplus & Lagrange (Linear) & $\mathrm{mol} / \mathrm{m}^{\wedge} 3$ & Concentration & Material & Domain 2 \\
\hline OH & Lagrange (Linear) & $\mathrm{mol} / \mathrm{m}^{\wedge} 3$ & Concentration & Material & Domain 2 \\
\hline Fc & Lagrange (Linear) & $\mathrm{mol} / \mathrm{m}^{\wedge} 3$ & Concentration & Material & Domain 2 \\
\hline FcH & Lagrange (Linear) & $\mathrm{mol} / \mathrm{m}^{\wedge} 3$ & Concentration & Material & Domain 2 \\
\hline Fcplus & Lagrange (Linear) & $\mathrm{mol} / \mathrm{m}^{\wedge} 3$ & Concentration & Material & Domain 2 \\
\hline MOHorg & Lagrange (Linear) & $\mathrm{mol} / \mathrm{m}^{\wedge} 3$ & Concentration & Material & Domain 2 \\
\hline O2 & Lagrange (Linear) & $\mathrm{mol} / \mathrm{m}^{\wedge} 3$ & Concentration & Material & Domain 2 \\
\hline HO2 & Lagrange (Linear) & $\mathrm{mol} / \mathrm{m}^{\wedge} 3$ & Concentration & Material & Domain 2 \\
\hline HO2minus & Lagrange (Linear) & $\mathrm{mol} / \mathrm{m}^{\wedge} 3$ & Concentration & Material & Domain 2 \\
\hline H2O2 & Lagrange (Linear) & $\mathrm{mol} / \mathrm{m}^{\wedge} 3$ & Concentration & Material & Domain 2 \\
\hline
\end{tabular}




\begin{tabular}{|c|c|c|}
\hline Weak expression & Integration frame & Selection \\
\hline $\begin{array}{l}\text {-d(Mplus,t)*test(Mplus)- } \\
\text { chds2.Dxx_Mplus*Mplusx*test(Mplusx) }\end{array}$ & Material & Domain 2 \\
\hline $\begin{array}{l}\text {-d(OH,t)*test(OH)- } \\
\text { chds2.Dxx_OH*OHx*test(OHx) }\end{array}$ & Material & Domain 2 \\
\hline -d(Fc,t)*test(Fc)-chds2.Dxx_Fc*Fcx*test(Fcx) & Material & Domain 2 \\
\hline $\begin{array}{l}\text {-d(FcH,t)*test(FcH)- } \\
\text { chds2.Dxx_FcH*FcHx*test(FcHx) }\end{array}$ & Material & Domain 2 \\
\hline $\begin{array}{l}\text {-d(Fcplus,t)*test(Fcplus)- } \\
\text { chds2.Dxx_Fcplus*Fcplusx*test(Fcplusx) }\end{array}$ & Material & Domain 2 \\
\hline $\begin{array}{l}\text {-d(MOHorg,t)*test(MOHorg)- } \\
\text { chds2.Dxx_MOHorg*MOHorgx*test(MOHorgx) }\end{array}$ & Material & Domain 2 \\
\hline -d(O2,t)*test(O2)-chds2.Dxx_O2*O2x*test(O2x) & Material & Domain 2 \\
\hline $\begin{array}{l}\text {-d(HO2,t)*test(HO2)- } \\
\text { chds2.Dxx_HO2*HO2x*test(HO2x) }\end{array}$ & Material & Domain 2 \\
\hline $\begin{array}{l}\text {-d(HO2minus,t)*test(HO2minus)- } \\
\text { chds2.Dxx_HO2minus*HO2minusx*test(HO2minu } \\
\mathrm{sx})\end{array}$ & Material & Domain 2 \\
\hline $\begin{array}{l}\text {-d(H2O2,t)*test(H2O2)- } \\
\text { chds2.Dxx_H2O2*H2O2x*test(H2O2x) }\end{array}$ & Material & Domain 2 \\
\hline -chds2.u*Mplusx*test(Mplus) & Material & Domain 2 \\
\hline chds2.cbf_Mplus*test(Mplus) & Material & Boundaries 2-3 \\
\hline -chds2.u*OHx*test(OH) & Material & Domain 2 \\
\hline chds2.cbf_OH*test(OH) & Material & Boundaries 2-3 \\
\hline -chds2.u*Fcx*test(Fc) & Material & Domain 2 \\
\hline chds2.cbf_Fc*test(Fc) & Material & Boundaries 2-3 \\
\hline -chds2.u*FcHx*test(FcH) & Material & Domain 2 \\
\hline chds2.cbf_FcH*test(FcH) & Material & Boundaries 2-3 \\
\hline -chds2.u*Fcplusx*test(Fcplus) & Material & Domain 2 \\
\hline
\end{tabular}




\begin{tabular}{|l|l|l|}
\hline Weak expression & Integration frame & Selection \\
\hline chds2.cbf_Fcplus*test(Fcplus) & Material & Boundaries 2-3 \\
\hline -chds2.u*MOHorgx*test(MOHorg) & Material & Domain 2 \\
\hline chds2.cbf_MOHorg*test(MOHorg) & Material & Boundaries 2-3 \\
\hline -chds2.u*O2x*test(O2) & Material & Domain 2 \\
\hline chds2.cbf_O2*test(O2) & Material & Boundaries 2-3 \\
\hline -chds2.u*HO2x*test(HO2) & Material & Domain 2 \\
\hline chds2.cbf_HO2*test(HO2) & Material & Boundaries 2-3 \\
\hline -chds2.u*HO2minusx*test(HO2minus) & Material & Domain 2 \\
\hline chds2.cbf_HO2minus*test(HO2minus) & Material & Boundaries 2-3 \\
\hline -chds2.u*H2O2x*test(H2O2) & Material & Domain 2 \\
\hline chds2.cbf_H2O2*test(H2O2) & Material & Boundaries 2-3 \\
\hline chds2.streamline & Material & Domain 2 \\
\hline chds2.crosswind & Material & Domain 2 \\
\hline
\end{tabular}

\subsubsection{No Flux 1}

\begin{tabular}{|l|l|l|l|l|}
-0.01 & -0.005 & 0 & 0.005 & 0.01
\end{tabular}

No Flux 1

Selection

\begin{tabular}{|l|l|}
\hline Geometric entity level & Boundary \\
\hline Selection & No boundaries \\
\hline
\end{tabular}




Equations
\[ -\mathbf{n} \cdot \mathbf{N}_{i}=0 \]
Settings
Settings
\begin{tabular}{|l|l|}
\hline Description & Value \\
\hline Apply for all species & Apply for all species \\
\hline
\end{tabular}

Used products

COMSOL Multiphysics

2.4.3 Initial Values 1

\begin{tabular}{llllll}
\hline 0 & 0.002 & 0.004 & 0.006 & 0.008 & 0.01
\end{tabular}

Initial Values 1

Selection

\begin{tabular}{|l|l|}
\hline Geometric entity level & Domain \\
\hline Selection & Domain 2 \\
\hline
\end{tabular}

Settings

Settings

\begin{tabular}{|l|l|}
\hline Description & Value \\
\hline Concentration & cMorgi \\
\hline Concentration & cOHorgi \\
\hline
\end{tabular}




\begin{tabular}{|l|l|}
\hline Description & Value \\
\hline Concentration & cDMFci \\
\hline Concentration & cFcHi \\
\hline Concentration & cDMFcplusi \\
\hline Concentration & cMOHi \\
\hline Concentration & cO2i \\
\hline Concentration & cHO2i \\
\hline Concentration & 0 \\
\hline Concentration & 0 \\
\hline
\end{tabular}

Used products

COMSOL Multiphysics

\subsubsection{Flux 1}

Flux 1

Selection

\begin{tabular}{|l|l|}
\hline Geometric entity level & Boundary \\
\hline Selection & Boundary 2 \\
\hline
\end{tabular}

Equations

$-\mathbf{n} \cdot \mathbf{N}_{i}=N_{0 j}$ 


\section{Settings}

Settings

\begin{tabular}{|l|l|}
\hline Description & Value \\
\hline Species Mplus & On \\
\hline Species OH & On \\
\hline Species Fc & Off \\
\hline Species FcH & Off \\
\hline Species Fcplus & On \\
\hline Species MOHorg & On \\
\hline Species O2 & Off \\
\hline Species HO2 & Off \\
\hline Species HO2minus & Off \\
\hline Species H2O2 & Off \\
\hline Inward flux & $\begin{array}{l}\{\mathrm{kb} * \text { Maq - kf*Mplus, kb3*OHaq - kf3*OH, 0, 0, -kf2*Fcplus }+ \\
\text { kb2*Fcplusaq, -kmohf*MOHorg + kmohb*MOH, 0, 0, 0, 0 }\}\end{array}$ \\
\hline Flux type & General inward flux \\
\hline
\end{tabular}

Used products

COMSOL Multiphysics

Variables

\begin{tabular}{|c|c|c|c|c|}
\hline Name & Expression & Unit & Description & Selection \\
\hline $\begin{array}{l}\text { chds2.cbf_ } \\
\text { Mplus }\end{array}$ & $\begin{array}{l}\text { Mplus*(chds2.u*chds2.nxmesh+ } \\
\text { chds2.v*chds2.nymesh+chds2.w } \\
\text { *chds2.nzmesh) }\end{array}$ & $\mathrm{mol} /\left(\mathrm{m}^{\wedge}{ }^{2 *} \mathrm{~s}\right)$ & $\begin{array}{l}\text { Convective } \\
\text { boundary } \\
\text { flux }\end{array}$ & Boundary 2 \\
\hline $\begin{array}{l}\text { chds2.cbf_- } \\
\mathrm{OH}\end{array}$ & $\begin{array}{l}\text { OH*(chds2.u*chds2.nxmesh+ch } \\
\text { ds2.v*chds2.nymesh+chds2.w*c } \\
\text { hds2.nzmesh) }\end{array}$ & $\mathrm{mol} /\left(\mathrm{m}^{\wedge} 2^{*} \mathrm{~s}\right)$ & $\begin{array}{l}\text { Convective } \\
\text { boundary } \\
\text { flux }\end{array}$ & Boundary 2 \\
\hline $\begin{array}{l}\text { chds2.cbf_- } \\
\text { Fc }\end{array}$ & $\begin{array}{l}\text { Fc*(chds2.u*chds2.nxmesh+chd } \\
\text { s2.v*chds2.nymesh+chds2.w*ch } \\
\text { ds2.nzmesh) }\end{array}$ & $\mathrm{mol} /\left(\mathrm{m}^{\wedge} 2^{*} \mathrm{~s}\right)$ & $\begin{array}{l}\text { Convective } \\
\text { boundary } \\
\text { flux }\end{array}$ & Boundary 2 \\
\hline
\end{tabular}




\begin{tabular}{|c|c|c|c|c|}
\hline Name & Expression & Unit & Description & Selection \\
\hline $\begin{array}{l}\text { chds2.cbf_ } \\
\text { FcH }\end{array}$ & $\begin{array}{l}\text { FcH*(chds2.u*chds2.nxmesh+ch } \\
\text { ds2.v*chds2.nymesh+chds2.w*c } \\
\text { hds2.nzmesh) }\end{array}$ & $\mathrm{mol} /\left(\mathrm{m}^{\wedge} 2^{*} \mathrm{~s}\right)$ & $\begin{array}{l}\text { Convective } \\
\text { boundary } \\
\text { flux }\end{array}$ & Boundary 2 \\
\hline $\begin{array}{l}\text { chds2.cbf_ } \\
\text { Fcplus }\end{array}$ & $\begin{array}{l}\text { Fcplus*(chds2.u*chds2.nxmesh+ } \\
\text { chds2.v*chds2.nymesh+chds2.w } \\
\text { *chds2.nzmesh) }\end{array}$ & $\mathrm{mol} /\left(\mathrm{m}^{\wedge} 2^{*} \mathrm{~s}\right)$ & $\begin{array}{l}\text { Convective } \\
\text { boundary } \\
\text { flux }\end{array}$ & Boundary 2 \\
\hline $\begin{array}{l}\text { chds2.cbf_ } \\
\text { MOHorg }\end{array}$ & $\begin{array}{l}\text { MOHorg*(chds2.u*chds2.nxmes } \\
\text { h+chds2.v*chds2.nymesh+chds2. } \\
\text { w*chds2.nzmesh) }\end{array}$ & $\mathrm{mol} /\left(\mathrm{m}^{\wedge} 2^{*} \mathrm{~s}\right)$ & $\begin{array}{l}\text { Convective } \\
\text { boundary } \\
\text { flux }\end{array}$ & Boundary 2 \\
\hline $\begin{array}{l}\text { chds2.cbf_ } \\
\text { O2 }\end{array}$ & $\begin{array}{l}\text { O2*(chds2.u*chds2.nxmesh+chd } \\
\text { s2.v*chds2.nymesh+chds2.w*ch } \\
\text { ds2.nzmesh) }\end{array}$ & $\mathrm{mol} /\left(\mathrm{m}^{\wedge} 2^{*} \mathrm{~s}\right)$ & $\begin{array}{l}\text { Convective } \\
\text { boundary } \\
\text { flux }\end{array}$ & Boundary 2 \\
\hline $\begin{array}{l}\text { chds2.cbf_ } \\
\text { HO2 }\end{array}$ & $\begin{array}{l}\text { HO2*(chds2.u*chds2.nxmesh+c } \\
\text { hds2.v*chds2.nymesh+chds2.w* } \\
\text { chds2.nzmesh) }\end{array}$ & $\mathrm{mol} /\left(\mathrm{m}^{\wedge} 2^{*} \mathrm{~s}\right)$ & $\begin{array}{l}\text { Convective } \\
\text { boundary } \\
\text { flux }\end{array}$ & Boundary 2 \\
\hline $\begin{array}{l}\text { chds2.cbf_ } \\
\text { HO2minus }\end{array}$ & $\begin{array}{l}\text { HO2minus*(chds2.u*chds2.nxm } \\
\text { esh+chds2.v*chds2.nymesh+chd } \\
\text { s2.w*chds2.nzmesh) }\end{array}$ & $\mathrm{mol} /\left(\mathrm{m}^{\wedge} 2^{*} \mathrm{~s}\right)$ & $\begin{array}{l}\text { Convective } \\
\text { boundary } \\
\text { flux }\end{array}$ & Boundary 2 \\
\hline $\begin{array}{l}\text { chds2.cbf_ } \\
\text { H2O2 }\end{array}$ & $\begin{array}{l}\text { H2O2*(chds2.u*chds2.nxmesh+ } \\
\text { chds2.v*chds2.nymesh+chds2.w } \\
\text { *chds2.nzmesh) }\end{array}$ & $\mathrm{mol} /\left(\mathrm{m}^{\wedge} 2^{*} \mathrm{~s}\right)$ & $\begin{array}{l}\text { Convective } \\
\text { boundary } \\
\text { flux }\end{array}$ & Boundary 2 \\
\hline
\end{tabular}

\section{Weak expressions}

\begin{tabular}{|l|l|l|}
\hline Weak expression & Integration frame & Selection \\
\hline (kb*Maq-kf*Mplus)*test(Mplus) & Material & Boundary 2 \\
\hline (kb3*OHaq-kf3*OH)*test(OH) & Material & Boundary 2 \\
\hline (-kf2*Fcplus+kb2*Fcplusaq)*test(Fcplus) & Material & Boundary 2 \\
\hline (-kmohf*MOHorg+kmohb*MOH)*test(MOHorg) & Material & Boundary 2 \\
\hline
\end{tabular}




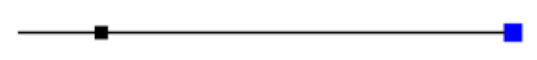

\begin{tabular}{l|lllll}
\hline 0 & 0.005 & 0.01 & 0.015 & 0.02 \\
\hline
\end{tabular}

Concentration 1

Selection

\begin{tabular}{|l|l|}
\hline Geometric entity level & Boundary \\
\hline Selection & Boundary 3 \\
\hline
\end{tabular}

Equations

$$
c_{i}=c_{0 j}
$$

Settings

Settings

\begin{tabular}{|l|l|}
\hline Description & Value \\
\hline Concentration & $\begin{array}{l}\{\text { cMorgi, cOHorgi, cDMFci, cFcHi, cDMFcplusi, cMOHi, cO2i, } \\
\text { cHO2i, 0, 0 }\}\end{array}$ \\
\hline Species Mplus & On \\
\hline Species OH & On \\
\hline Species Fc & On \\
\hline Species FcH & On \\
\hline Species Fcplus & On \\
\hline Species MOHorg & On \\
\hline Species O2 & On \\
\hline
\end{tabular}




\begin{tabular}{|l|l|}
\hline Description & Value \\
\hline Species HO2 & On \\
\hline Species HO2minus & On \\
\hline Species H2O2 & On \\
\hline Apply reaction terms on & All physics (symmetric) \\
\hline Use weak constraints & Off \\
\hline
\end{tabular}

Used products

COMSOL Multiphysics

Variables

\begin{tabular}{|l|l|l|l|l|}
\hline Name & Expression & Unit & Description & Selection \\
\hline chds2.c0_Mplus & cMorgi & $\mathrm{mol} / \mathrm{m}^{\wedge} 3$ & Concentration & Boundary 3 \\
\hline chds2.c0_OH & cOHorgi & $\mathrm{mol} / \mathrm{m}^{\wedge} 3$ & Concentration & Boundary 3 \\
\hline chds2.c0_Fc & cDMFci & $\mathrm{mol} / \mathrm{m}^{\wedge} 3$ & Concentration & Boundary 3 \\
\hline chds2.c0_FcH & cFcHi & $\mathrm{mol} / \mathrm{m}^{\wedge}$ 3 & Concentration & Boundary 3 \\
\hline chds2.c0_Fcplus & cDMFcplusi & $\mathrm{mol} / \mathrm{m}^{\wedge} 3$ & Concentration & Boundary 3 \\
\hline chds2.c0_MOHorg & cMOHi & $\mathrm{mol} / \mathrm{m}^{\wedge} 3$ & Concentration & Boundary 3 \\
\hline chds2.c0_O2 & cO2i & $\mathrm{mol} / \mathrm{m}^{\wedge} 3$ & Concentration & Boundary 3 \\
\hline chds2.c0_HO2 & cHO2i & $\mathrm{mol} / \mathrm{m}^{\wedge} 3$ & Concentration & Boundary 3 \\
\hline chds2.c0_HO2minus & 0 & $\mathrm{~mol} / \mathrm{m}^{\wedge} 3$ & Concentration & Boundary 3 \\
\hline chds2.c0_H2O2 & 0 & $\mathrm{~mol} / \mathrm{m}^{\wedge} 3$ & Concentration & Boundary 3 \\
\hline
\end{tabular}

\section{Shape functions}

\begin{tabular}{|l|l|l|l|}
\hline Constraint & Constraint force & Shape function & Selection \\
\hline -Mplus+chds2.c0_Mplus & $\begin{array}{l}\text { test(- } \\
\text { Mplus+chds2.c0_Mplus) }\end{array}$ & Lagrange (Linear) & Boundary 3 \\
\hline -OH+chds2.c0_OH & test(-OH+chds2.c0_OH) & Lagrange (Linear) & Boundary 3 \\
\hline -Fc+chds2.c0_Fc & test(-Fc+chds2.c0_Fc) & Lagrange (Linear) & Boundary 3 \\
\hline -FcH+chds2.c0_FcH & test(-FcH+chds2.c0_FcH) & Lagrange (Linear) & Boundary 3 \\
\hline
\end{tabular}




\begin{tabular}{|c|c|c|c|}
\hline Constraint & Constraint force & Shape function & Selection \\
\hline -Fcplus+chds2.c0_Fcplus & $\begin{array}{l}\text { test(- } \\
\text { Fcplus+chds2.c0_Fcplus) }\end{array}$ & Lagrange (Linear) & Boundary 3 \\
\hline $\begin{array}{l}- \\
\text { MOHorg+chds2.c0_MOH } \\
\text { org }\end{array}$ & $\begin{array}{l}\text { test(- } \\
\text { MOHorg+chds2.c0_MOH } \\
\text { org) }\end{array}$ & Lagrange (Linear) & Boundary 3 \\
\hline -O2+chds2.c0_O2 & test(-O2+chds2.c0_O2) & Lagrange (Linear) & Boundary 3 \\
\hline -HO2+chds2.c0_HO2 & $\begin{array}{l}\text { test(- } \\
\text { HO2+chds2.c0_HO2) }\end{array}$ & Lagrange (Linear) & Boundary 3 \\
\hline $\begin{array}{l}- \\
\text { HO2minus+chds2.c0_HO } \\
\text { 2minus }\end{array}$ & $\begin{array}{l}\text { test(- } \\
\text { HO2minus+chds2.c0_HO } \\
\text { 2minus) }\end{array}$ & Lagrange (Linear) & Boundary 3 \\
\hline -H2O2+chds2.c0_H2O2 & $\begin{array}{l}\text { test(- } \\
\text { H2O2+chds2.c0_H2O2) }\end{array}$ & Lagrange (Linear) & Boundary 3 \\
\hline
\end{tabular}

\subsubsection{Reactions 1}

\begin{tabular}{llllll}
\hline 0 & 0.002 & 0.004 & 0.006 & 0.008 & 0.01
\end{tabular}

Reactions 1

Selection

\begin{tabular}{|l|l|}
\hline Geometric entity level & Domain \\
\hline Selection & Domain 2 \\
\hline
\end{tabular}


Equations

$$
\frac{\partial c_{i}}{\partial t}+\nabla \cdot\left(-D_{i} \nabla c_{i}\right)+\mathbf{u} \cdot \nabla c_{i}=R_{i}
$$

\section{Settings}

Settings

\begin{tabular}{|c|c|}
\hline Description & Value \\
\hline ression & 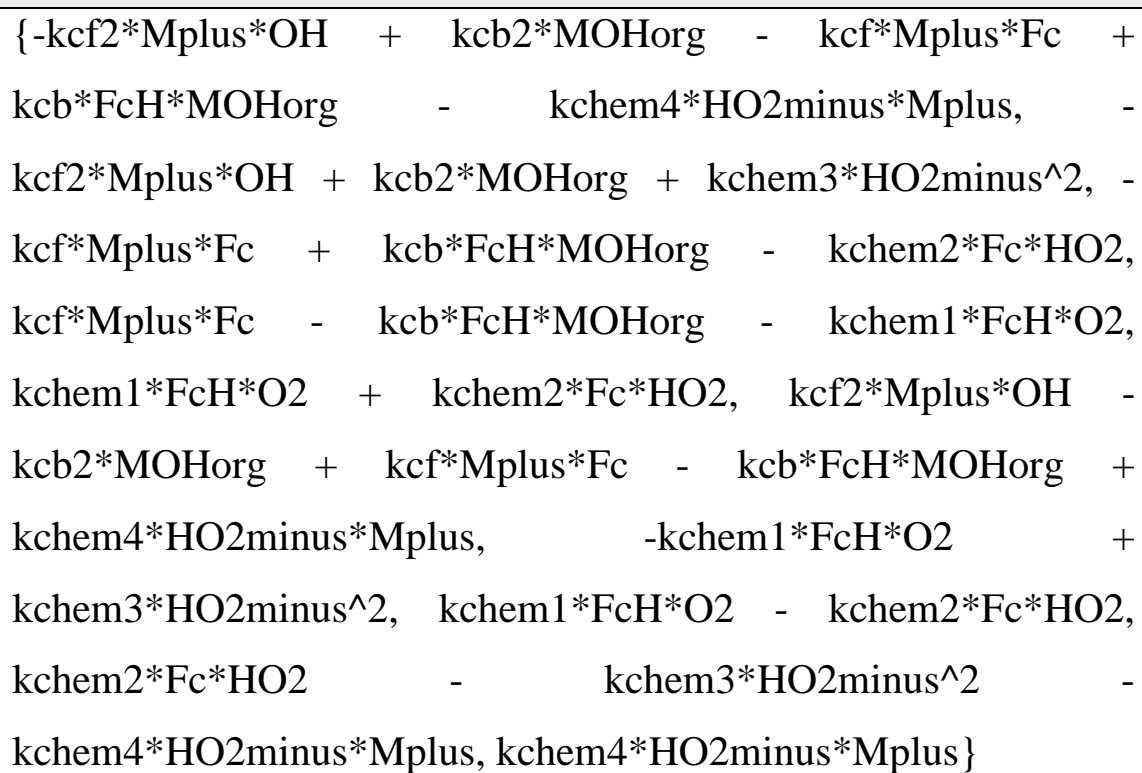 \\
\hline
\end{tabular}

\section{Used products}

\section{COMSOL Multiphysics}

\section{Variables}

\begin{tabular}{|c|c|c|c|c|}
\hline Name & Expression & Unit & Description & Selection \\
\hline $\begin{array}{l}\text { chds2.R_ } \\
\text { Mplus }\end{array}$ & $\begin{array}{l}\text {-kcf2*Mplus*OH+kcb2*MOHorg- } \\
\text { kcf*Mplus*Fc+kcb*FcH*MOHorg } \\
\text {-kchem4*HO2minus*Mplus }\end{array}$ & $\mathrm{mol} /\left(\mathrm{m}^{\wedge} 3^{*} \mathrm{~s}\right)$ & $\begin{array}{l}\text { Total rate } \\
\text { expression }\end{array}$ & Domain 2 \\
\hline $\begin{array}{l}\text { chds2.R_ } \\
\mathrm{OH}\end{array}$ & $\begin{array}{l}\text { - } \\
\text { kcf2*Mplus*OH+kcb2*MOHorg+ } \\
\text { kchem3*HO2minus^2 }\end{array}$ & $\mathrm{mol} /\left(\mathrm{m}^{\wedge} 3^{*} \mathrm{~s}\right)$ & $\begin{array}{l}\text { Total rate } \\
\text { expression }\end{array}$ & Domain 2 \\
\hline $\begin{array}{l}\text { chds2.R_F } \\
\text { c }\end{array}$ & $\begin{array}{l}\text { - } \\
\text { kcf*Mplus*Fc+kcb*FcH*MOHorg } \\
\text {-kchem2*Fc*HO2 }\end{array}$ & $\mathrm{mol} /\left(\mathrm{m}^{\wedge} 3^{*} \mathrm{~s}\right)$ & $\begin{array}{l}\text { Total rate } \\
\text { expression }\end{array}$ & Domain 2 \\
\hline
\end{tabular}




\begin{tabular}{|c|c|c|c|c|}
\hline Name & Expression & Unit & Description & Selection \\
\hline $\begin{array}{l}\text { chds2.R_F } \\
\text { cH }\end{array}$ & $\begin{array}{l}\text { kcf*Mplus*Fc- } \\
\text { kcb*FcH*MOHorg- } \\
\text { kchem } 1 * \mathrm{FcH}^{*} \mathrm{O} 2\end{array}$ & $\mathrm{~mol} /\left(\mathrm{m}^{\wedge} 3^{*} \mathrm{~s}\right)$ & $\begin{array}{l}\text { Total rate } \\
\text { expression }\end{array}$ & Domain 2 \\
\hline $\begin{array}{l}\text { chds2.R_F } \\
\text { cplus }\end{array}$ & $\begin{array}{l}\text { kchem } 1 * \mathrm{FcH}^{*} \mathrm{O} 2+\mathrm{kchem} 2 * \mathrm{Fc} * \mathrm{HO} \\
2\end{array}$ & $\mathrm{~mol} /\left(\mathrm{m}^{\wedge} 3^{*} \mathrm{~s}\right)$ & $\begin{array}{l}\text { Total rate } \\
\text { expression }\end{array}$ & Domain 2 \\
\hline $\begin{array}{l}\text { chds2.R_ } \\
\text { MOHorg }\end{array}$ & $\begin{array}{l}\text { kcf2*Mplus*OH- } \\
\text { kcb2*MOHorg+kcf*Mplus*Fc- } \\
\text { kcb*FcH*MOHorg+kchem4*HO2 } \\
\text { minus*Mplus }\end{array}$ & $\mathrm{mol} /\left(\mathrm{m}^{\wedge} 3^{*} \mathrm{~s}\right)$ & $\begin{array}{l}\text { Total rate } \\
\text { expression }\end{array}$ & Domain 2 \\
\hline $\begin{array}{l}\text { chds2.R_ } \\
\text { O2 }\end{array}$ & $\begin{array}{l}\text { - } \\
\text { kchem } 1 * \mathrm{FcH}^{*} \mathrm{O} 2+\mathrm{kchem} 3 * \mathrm{HO} 2 \mathrm{~m} \\
\text { inus }^{\wedge} 2\end{array}$ & $\mathrm{~mol} /\left(\mathrm{m}^{\wedge} 3^{*} \mathrm{~s}\right)$ & $\begin{array}{l}\text { Total rate } \\
\text { expression }\end{array}$ & Domain 2 \\
\hline $\begin{array}{l}\text { chds2.R_ } \\
\text { HO2 }\end{array}$ & $\begin{array}{l}\text { kchem } 1 * \mathrm{FcH}^{*} \mathrm{O} 2- \\
\text { kchem } 2 * \mathrm{Fc}^{*} \mathrm{HO} 2\end{array}$ & $\mathrm{~mol} /\left(\mathrm{m}^{\wedge} 3^{*} \mathrm{~s}\right)$ & $\begin{array}{l}\text { Total rate } \\
\text { expression }\end{array}$ & Domain 2 \\
\hline $\begin{array}{l}\text { chds2.R_ } \\
\text { HO2minu } \\
\text { s }\end{array}$ & $\begin{array}{l}\text { kchem2*Fc*HO2- } \\
\text { kchem3*HO2minus^2- } \\
\text { kchem4*HO2minus*Mplus }\end{array}$ & $\mathrm{mol} /\left(\mathrm{m}^{\wedge} 3^{*} \mathrm{~s}\right)$ & $\begin{array}{l}\text { Total rate } \\
\text { expression }\end{array}$ & Domain 2 \\
\hline $\begin{array}{l}\text { chds2.R_ } \\
\text { H2O2 }\end{array}$ & kchem4*HO2minus*Mplus & $\mathrm{mol} /\left(\mathrm{m}^{\wedge} 3^{*} \mathrm{~s}\right)$ & $\begin{array}{l}\text { Total rate } \\
\text { expression }\end{array}$ & Domain 2 \\
\hline
\end{tabular}

\section{Weak expressions}

\begin{tabular}{|l|l|l|}
\hline Weak expression & Integration frame & Selection \\
\hline $\begin{array}{l}(-\mathrm{kcf} 2 * \text { Mplus*OH+kcb2*MOHorg- } \\
\text { kcf*Mplus*Fc+kcb*FcH*MOHorg- } \\
\text { kchem4*HO2minus*Mplus)*test(Mplus) }\end{array}$ & Material & Domain 2 \\
\hline $\begin{array}{l}(- \\
\text { kcf2*Mplus*OH+kcb2*MOHorg+kchem3*HO2minus^2 } \\
)^{*} \text { test(OH) }\end{array}$ & Material & \\
\hline $\begin{array}{l}\left(-\mathrm{kcf} * \mathrm{Mplus} * \mathrm{Fc}+\mathrm{kcb} * \mathrm{FcH}{ }^{*} \mathrm{MOH} \text { Horg- }\right. \\
\mathrm{kchem} 2 * \mathrm{Fc} * \mathrm{HO} 2) * t e s t(\mathrm{Fc})\end{array}$ & Material & \\
\hline
\end{tabular}




\begin{tabular}{|c|c|c|}
\hline Weak expression & Integration frame & Selection \\
\hline $\begin{array}{l}\text { (kcf*Mplus*Fc-kcb*FcH*MOHorg- } \\
\text { kchem } 1 * \text { FcH*O2)*test(FcH) }\end{array}$ & Material & Domain 2 \\
\hline (kchem1*FcH*O2+kchem2*Fc*HO2)*test(Fcplus) & Material & Domain 2 \\
\hline $\begin{array}{l}\text { (kcf2*Mplus*OH-kcb2*MOHorg+kcf*Mplus*Fc- } \\
\text { kcb*FcH*MOHorg+kchem4*HO2minus*Mplus)*test(M } \\
\text { OHorg) }\end{array}$ & Material & Domain 2 \\
\hline$\left(-\mathrm{kchem} 1 * \mathrm{FcH}^{*} \mathrm{O} 2+\mathrm{kchem} 3 * \mathrm{HO} 2 \mathrm{minus}^{\wedge} 2\right)^{*} \operatorname{test}(\mathrm{O} 2)$ & Material & Domain 2 \\
\hline (kchem1*FcH*O2-kchem2*Fc*HO2)*test(HO2) & Material & Domain 2 \\
\hline $\begin{array}{l}\text { (kchem2*Fc*HO2-kchem3*HO2minus^2- } \\
\text { kchem4*HO2minus*Mplus)*test(HO2minus) }\end{array}$ & Material & Domain 2 \\
\hline kchem4*HO2minus*Mplus*test(H2O2) & Material & Domain 2 \\
\hline
\end{tabular}

\subsection{Mesh 1}

Mesh statistics

\begin{tabular}{|l|l|}
\hline Property & Value \\
\hline Minimum element quality & 1.0 \\
\hline Average element quality & 1.0 \\
\hline Edge elements & 4000 \\
\hline Vertex elements & 3 \\
\hline
\end{tabular}




\begin{tabular}{ll|l|l|l|l|}
-1 & -0.5 & 0 & 0.5 & 1
\end{tabular}

Mesh 1

2.5.1 Size (size)

Settings

\begin{tabular}{|l|l|}
\hline Name & Value \\
\hline Maximum element size & $5 \mathrm{e}-6$ \\
\hline Minimum element size & $6.0 \mathrm{E}-6$ \\
\hline Curvature factor & 0.3 \\
\hline Maximum element growth rate & 1.3 \\
\hline Custom element size & Custom \\
\hline
\end{tabular}

2.5.2 Edge 1 (edg1)

Selection

\begin{tabular}{|l|l|}
\hline Geometric entity level & Remaining \\
\hline
\end{tabular} 